\title{
THE GENDERED EXPERIENCE
}

\section{OF VOICE IN CANADIAN RADIO AND BEYOND: TORONTO, CANADA}

\author{
by \\ Anastasia (Stacey) Copeland \\ BA, Ryerson University, Toronto, Ontario, 2014 \\ A thesis presented to Ryerson University and York University \\ in partial fulfillment of the requirements for the degree of \\ Master of Arts \\ in the program of \\ Communication \& Culture
}

Toronto, Ontario, Canada, 2017

CStacey Copeland, 2017 


\section{AUTHOR'S DECLARATION FOR ELECTRONIC SUBMISSION OF A THESIS}

I hereby declare that I am the sole author of this thesis. This is a true copy of the thesis, including any required final revisions, as accepted by my examiners.

I authorize Ryerson University to lend this thesis to other institutions or individuals for the purpose of scholarly research.

I further authorize Ryerson University to reproduce this thesis by photocopying or by other means, in total or in part, at the request of other institutions or individuals for the purpose of scholarly research.

I understand that my thesis may be made electronically available to the public. 


\begin{abstract}
The Gendered Experience of Voice in Canadian Radio and Beyond: Toronto, Canada Anastasia (Stacey) Copeland

Master of Arts, Communication and Culture

Ryerson University, 2017
\end{abstract}

Feminist media scholars have historically centered gender and identity on the body and visual texts, with the voice exercised as metaphor - immaterial or interpreted solely as the words spoken. Representative of agency, the voice gets defined as what is being said rather than how one is saying it. My thesis addresses this gap through an earoriented analysis of women's voice within the Canadian radio and podcasting industry. Centred on the experiences of individual women in Toronto's broadcast soundscape, I bring a feminist phenomenological approach to my work to explore the intersection of voice as both material sound -an extension of the body and thus individual identities- and the weight of the women's voice as politically and historically coded. I aim to expand my work beyond the individual experiences of the women within the broadcast industry and into the broader discourse surrounding gendered representation for the future of our Canadian media soundscape. 


\section{ACKNOWLEDGMENTS}

The process of writing a master's thesis is not one you can navigate alone. There are many magnificent people who have contributed their time and support along the way. First off a big thank you to all the amazing radio women of Toronto who took the time to share their experiences and voices through this project. Without your lived experiences and true passion for what you do, this research wouldn't exist. Next I must thank my supervisor Anne MacLennan. We met on program orientation day where I nervously divulged my interests in radio studies and women's media history. Anne has been my ideal mentor ever since, challenging me to attend conferences, present my work internationally and to be the best version of myself. A big thank you also goes to Paul Moore for his invaluable guidance in navigating the many twists and turns of being a graduate student. I also must give thanks to my committee members, Susan Driver and Art Blake. Early on into my time at Ryerson and York, Susan gave me the encouragement I needed to pursue research into the experience of gendered voice from a feminist perspective. Through a directed reading on sound studies, Art brought new perspective to my work and introduced me to further sound theories, opening up new avenues for my future graduate research. Last but certainly not least, my wonderful partner and fiancée Katie Chester. Your strength and continued support during these past two years has helped keep my eyes on the stars and my feet on the ground. A big final thanks to my parents Pat and Steve, friends, family and of course my colleagues in ComCult and the RTA School of Media who have gifted me the love and support essential to the pursuit of an academic future. 


\section{TABLE OF CONTENTS}

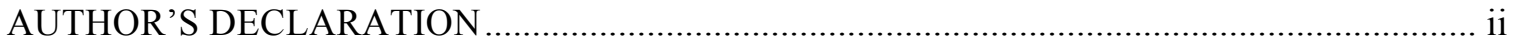

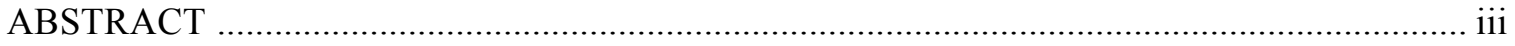

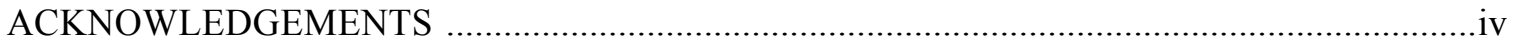

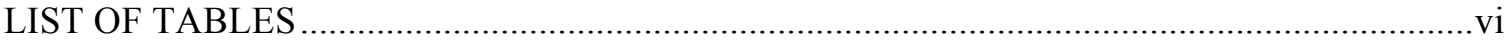

INTRODUCTION. Gendered Voice in Our Radio Soundscape ....................................1

CHAPTER ONE. Theoretical Framework......................................................................15

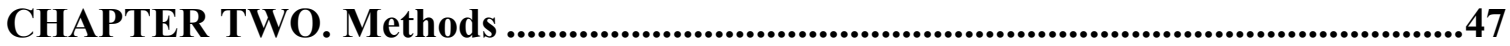

CHAPTER THREE. The 'Ideal' Radio Voice ..................................................................61

Pitch and Perception of Gendered Vocal Performativity

CHAPTER FOUR. Gendered Experience of Soundwork.........................................75

Feminine and Female Voices in Sound Media

CHAPTER FIVE. Radio Voice as an Extension of Self .............................................91 Vocal Self-Identity and 'Authentic' Presentation

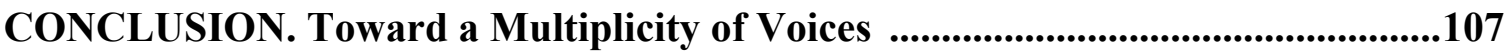

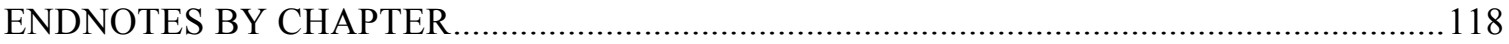

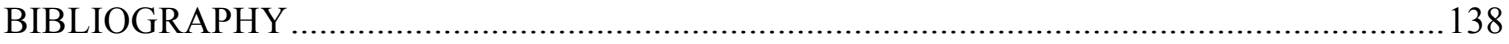




\section{LIST OF TABLES}

3.1 Top 4 Traits of the 'Ideal' Radio Voice.....

3.2 Top 5 Traits of Participants' Own Radio Voice .

3.3 Pitch Analysis produced in Praat Software. .68

3.4 NY Speech \& Voice Lab Pitch Chart (2014) .68

3.5 Most 'Undesired' Radio Voice .72 


\section{INTRODUCTION}

\section{Gendered Voice in Our Radio Soundscape}

A woman's voice on the radio is a simple phrase yet one that carries a heavy historical and cultural weight. The amplified voice holds a unique power, to inspire, to communicate, and to connect. In our hyper-visual world from television to smartphones, there is something inherently authentic and deeply human about the embodied voice amplified, electrified and extended through sound media. In Speaking for Themselves: How Radio Brought Women into The Public Sphere, scholar Donna Halper discusses the rise of women in radio in the late 1910 s, early 1920 s. ${ }^{1}$ Despite these gains, by the mid1920s societal norms left many voices muted or simply unheard in the media soundscape. Women's voices amplified through radio became public controversy. Perhaps it was the intimacy of the medium and backward gender divides that produced uneasiness and distaste for women's voice over the air - by women and men alike - particularly when women performed as announcers.

Halper highlights a letter from a 1920s record store owner to the editors of Radio Broadcast magazine asserting, "women needed to be seen to be appreciated, and just hearing them would not be enough to hold the audience's interest." ${ }^{2}$ This view of the woman's voice as unauthoritative and lacking of power, often limited the voice of women on radio to popular entertainment such as singing and radio dramas rather than positions of influence within radio talk formats. 
The misogyny and gendered biases highlighted in Halper's study are part of our complex gendered radio history, but the same patriarchal structure continues to dominate the broadcast industry today. A 2015 analysis of male and female voices in Ireland's news and current affairs radio industry and the amount of time each is heard over the three radio stations examined, shows a breakdown of $72 \%$ male and only $28 \%$ female voices. ${ }^{3}$ Although a comparable study has yet to be published in Canada, similar numbers can be estimated based on the 2015 findings from the Global Media Monitoring Project, which found that "women make up only $24 \%$ of the persons heard, read about or seen in newspaper, television and radio news", the same numbers found in their 2010 study. ${ }^{4}$ With 27.4 million Canadians listening to the radio every week and countless more engaging with podcasting and audio-on-demand, ${ }^{5}$ the gendered radio soundscape in Canada holds a crucial role in the ways that women's voices are presented, perceived and decoded. As the largest city centre in the country, Toronto, Ontario stands as a significant sampling of Canada's diverse population for the study of gendered radio voice in our Canadian soundscape. While the voices we hear in aural media change and as the content and distribution of those voices swing between terrestrial radio and digital media, further research into the simultaneously electrified and embodied gendered voice along with the experiences of women in soundwork must be done before we can begin to understand the effects that aural representations of women in media have on a larger socio-cultural scale. Through my thesis I explore the following questions:

- What does the relationship between gender representations, the material embodied voice and perceptions of female voice within sound-driven media (podcasting/radio) say about the representation of women in this industry? 
- How does the dramaturgical and on-air voice reflect or reject 'authentic' identity and concepts of self?

- Are broadcast voices experiencing an aural shift away from the traditional 'masculine' radio voice?

- What do these findings mean within the broader conversation surrounding diversity and gender equality in Canadian media?

There is always a certain paradox involved in writing visual text about embodied voice and sound. ${ }^{6}$ This contradicting notion, and certainly the historical apprehensions or 'deafness' toward a study of the voice across disciplines from media studies to feminist philosophy, has created a gap in academic cultural discourse up until the recent flow of interest in radio and sound studies. Feminist media scholars have historically centered gender and identity on the body and visual texts, with the voice exercised as metaphor immaterial or interpreted solely as the words spoken. As sound philosopher, Adriana Cavarero puts it, "The voice is sound not speech, but speech constitutes its essential destination." ${ }^{, 7}$ Representative of agency, the voice often finds itself defined as what is being said rather than how one is saying it. My thesis addresses this gap through a qualitative analysis of women's voice within traditional radio and digital based ondemand soundwork as experienced by the broadcasters themselves. I expand beyond the individual experiences of the women-identified peoples I interview within the Toronto soundwork industry and into the broader discourse surrounding female and 'feminine' representation in Canadian media. 


\section{Framing an Aural Theoretical Approach}

Through a feminist phenomenological approach, we can explore the intersection of voice as material sound - an extension of the body and thus individual identities - and the weight of the woman's voice as politically and historically coded. Voice is an integral part of our identity and our representation of self within our socio-cultural world on and off the microphone. Sound and voice are sonic locations where the categories of 'male' and 'female' and 'masculine' and 'feminine' are constituted and reconstituted through repetition. In the words of radio historian Christine Ehrick, our voices are by extension "the ways that power, inequality and agency might be expressed via sound". ${ }^{8}$ In Chapter One, I outline the theoretical framework and key literature which ground my critical study of gendered radio voice. My work sits within a growing intersection between the fields of sound studies and radio studies within our digital era. I choose to use the term 'soundworks' put forward by Michele Hilmes in "The New Materiality of Radio", "creative/constructed aural texts that employ the basic sonic elements of speech, music and noise; this excludes the field normally encompassed by the term 'music,' though of course, the boundaries are anything but clear. Typically speech is the dominant aspect of

soundwork, with music and noise secondary." ${ }^{, 9}$ f further the broad potentiality of this term to include talk segments on music-based stations as well as chat-genre podcasting. Our definition and understanding of 'radio' is experiencing an identity crisis in our digital age. 'Soundwork' becomes a significantly useful term to draw attention to the ways in which radio is changing. It offers up a new means to discuss alternative terms for the current conversational and talk-based works being produced in sound media in relation to digital access, cultural norms and the blurring of traditional radio broadcasting formats. 
The aural shift in gendered voice within our digital era is discussed by radio scholar Jason Loviglio in relation to the female voices of National Public Radio (NPR) in the United States. ${ }^{10}$ The aural attributes common to the female radio voice of NPR being low in pitch, "pronounced lack of pitch variance, a kind of disciplined, flat monotone delivery, with few pitch shifts" shows that women's voices on air tend to conform to the masculine radio ideal. ${ }^{11}$ Hilmes and Loviglio spark discussion around the aural shift in soundwork in relation to women's voice, but more must be done to delve into the effect these aurally gendered performances have on the experiences, identities and authenticities of the women behind the microphone. The gendered voice within the fields of feminist media studies holds a robust history, but as Ehrick points out in her gendered soundscape work, sound studies and phenomenology of voice seldom intersect with the study of women's history. ${ }^{12}$ I turn to the phenomenological frameworks of Don Ihde to re-center the experience of voice and of listening in the study of communication and culture. Listening and Voice presents a guide to the phenomenological study of sound through the relationships between language, voice, music, religion and silence. ${ }^{13}$ Originally written in 1976, this early publication offers a detailed phenomenological foundation for listening and voice guided by his understanding and expansions on the philosophies of Husserl and Heidegger. Although Ihde's text brings forward a refreshingly embodied framework for the study of sound and voice, I require a feminist theoretical approach to bring sound phenomenology into conversation with gendered experience and identity work. Judith Butler's Gender Trouble in conversation with more contemporary works by feminist sound scholars Annette Schlichter and Linda Fisher bring the theory of performativity into the realm of the embodied radio voice within our gendered soundscape. ${ }^{14}$ 
The foundations of sound studies in relation to aural analysis of the acoustic environment of radio can be credited to R. Murray Schafer's The Soundscape, where the term soundcape used throughout my work originates. Soundscape is defined by Schafer as a sonic environment "with emphasis on the way it is perceived and understood by the individual, or by a society." ${ }^{\prime 15}$ The term may refer to actual acoustic ecologies such as a city or a forest, or to abstract constructions such as musical compositions and radio documentary, particularly when considered as an artificial environment. In this work I refer to Toronto's radio soundscape. This particular soundscape is made up of the many radio broadcasts and podcasts - including their producers and listeners - within the Greater Toronto Area and must be thought of in relation to the particular time period and political contexts at play that influence today's overall sound. As a study focused on the radio soundscape as a gendered experience, feminist radio perspective in my thesis is rooted in the works of Caroline Mitchell and Michelle Hilmes, with Christine Ehrick's work bridging the gap between gender studies, sound studies and radio studies.

These key texts stand out as foundational to my theoretical framework and interdisciplinary understanding of the sonorous embodied gendered voice in soundwork. The feminist philosophical theories on voice presented by Adriana Cavarero are essential to my understanding of the power of embodied voice while Andrew M. Kimbrough and Anne Karpf provide an accessible historical perspective on voice across disciplines into the twentieth century. To discuss embodied voice in our modern digital culture, I draw on the work of Madsen and Potts in Voice: Vocal Aesthetics in Digital Arts and Media. I have also included a brief section on scientific and linguistic research of influence to the study of vocal quality and pitch featuring the work of Pittam and Gallois, Valentine and 
Saint Damian, Davies and Golderg, and Warhurst, McCabe, and Madill. The area of sociolinguistics has a rich history of voice studies that works with the material voice through analysis of pitch, paralanguage and more. Feminist phenomenology brought into conversation with linguistic tools and perspectives on vocality offer a new approach to the study of gendered radio voice as both embodied electrified voice and embodied human experience.

\section{Sounding Critical Methods}

Through a multidisciplinary approach centered on the experiences of individual women in radio and podcasting, this study works to uncover within these primarily auraldriven media; the relationship between gender representation and the material voice, shared industry perceptions of female voice, and how dramaturgical and on-air voice reflects or rejects 'authentic' identity and concepts of self. In 'Chapter Two' I outline the research methodologies required to fuse together the interdisciplinary theories foundational to my work through a focus on the value of experience and the sonic qualities of the material voice. Semi-structured interviews with ten woman-identified Toronto radio broadcast and podcast talent create the basis for this work. As noted by Ihde, "experience cannot be questioned alone or in isolation but must be understood ultimately in relation to its historical and cultural embeddedness. ${ }^{16}$ In this vein, experience takes centre stage in my methodological approach through the form of interview material to be contextualized in relation to gender politics, women's voices and the historical (and contemporary) patriarchal sounds of Toronto's radio soundscape. 
The self and the listener always experience the human voice as simultaneously embodied (material) and constructed (cultural). To approach this feminist phenomenological experience, aural discourse analysis is brought into conversation with pitch analysis of select interview materials. The value of discourse analysis in relation to aural analysis of interview materials cannot be ignored as the relationship between speech and voice are crucial to the encoding and decoding of meaning. It is through feminist discourse analysis as utilized by Rosalind Gill that key themes of authentic voice, selfperception, gendered experience and their corresponding terms arose through the transcription and coding process. ${ }^{17}$ While the self-perception and experiences of Toronto's soundwork women play lead role in my work, the aural experience of the voice is subjective and experienced differently by the speaker and the listener. With this in mind, the observation that women's voices in Western culture are changing and getting deeper ${ }^{18}$ cannot simply be left unexplored as a sonically material aspect to be analyzed through not only vocal self-perception but computer assisted pitch analysis. As Fisher put it so eloquently, the "importance of the materiality of the voices with which we speak and the vocalic experience as both subjective and intersubjective" ${ }^{\text {19 }}$ constructs voice as a complex social and cultural product. The analysis of pitch as an interdisciplinary approach to the gendered material voice in radio allows us to compare and contrast heteronormative notions of masculine and feminine voice in relation to perceptions of the 'ideal' radio voice in our modern soundscape. 


\section{'Ideal' Radio Voice: Pitch and Perceptions of Gendered Vocal Performativity}

Across the globe from Japan to Sweden to Canada, the gender norms and expectations of the 'ideal' radio voice are a direct reflection of that society's political and cultural framework. Although our perceptions of what is deemed to be an 'ideal' radio voice are changing along with increased access to a multiplicity of voices through the rise of podcasting, women's voices in public space continue to be policed, subject to higher scrutiny and judgment than their male counterparts. As Christine Ehrick writes, "as we learn to become more 'ear-oriented' gender scholars, we come to perceive power, oppression and agency in entirely new ways. ${ }^{20}$ Chapter Three positions modern concepts of the 'ideal' radio voice - brought forward by Toronto's female radio and podcasting talent - within our larger political soundscape to address the relationships between the gendered material voice and patriarchal power.

Pitch analysis in conversation with the experiences of women in soundwork brings the material embodied voice back into radio studies and offers an interdisciplinary approach to the question of whether or not women's voices are getting deeper or we are moving toward a multiplicity of sonic diversity. Pitch is the quality of voice (or sound) in relation to the highness or lowness of its tone tied to the rate of vibrations producing it. It is considered paralanguage - nonlinguistic components of speech communication including pitch, intonation and expression which structure our concepts of gender, culture, sexuality and class through repeated aural recognition. Auditory differentiation of gendered voice is a complex neurological process within the auditory cortex of the human brain, ${ }^{21}$ but when considered in dialogue with concepts of gender performativity as 
conceived by Judith Butler, we must approach these perceptions as construction through repetition within our social and cultural histories. Traits of the contemporary 'ideal' radio voice lead us toward an 'un-gendering' of vocal performativity while perceptions of select women's own voices on air tilt us back toward the gendered biases of the past. The experiences of women in Toronto's soundwork industry on 'ideal' radio voice are required to develop a greater understanding of the socio-cultural frameworks that shape our perceptions of 'masculine' and 'feminine' voice influenced in turn by their media representations. How we can move toward a more equitable broadcast soundscape and what that future may sound like is unclear but clues are hidden within the pitch and perceptions of gendered radio voice.

\section{Gendered Experience in Soundwork: Feminine and Female Voices of Sound Media}

Perception of female voice in radio has a vast and complex history of bias and scrutiny. As a result, the 'ideal' radio voice has long stood at a troubling gendered impasse. In Radio Voices, Hilmes outlines three historical events of the 1920s and 30s, including a heated public debate in American broadcast that led to the segregation of the women's voice to daytime hours in North America and the privileging of the 'deep' or 'masculine' voice for the primetime radio announcer. ${ }^{22}$ Through the work of feminist radio historians like Hilmes, women's voices have been written back into our historical radio soundscapes across the globe. Yet perceptions of 'feminine' high pitched voices on air continue to be perceived as 'undesired' for professional broadcast, while lower more 'masculine' coded voices continue to dominate. 
An investigation into this sonic phenomenon is presented in 'Chapter Four' through the experiences of Toronto soundwork women. The gendered prejudice and fears of the radio industry brought to light by Gill's UK study over twenty years ago still permeate our broadcast soundscape. ${ }^{23}$ 'Uptalk', 'vocal fry', and higher pitched voices receive particular note as traits discriminated against in relation to the female or 'feminine' voice on air. ${ }^{24}$ The gender inequality experienced by particular women of soundwork is normalized through the continual repetition of stereotypes related to the 'feminine' voice and the continued domination of our soundscape by more masculine coded presentation. Every voice you hear is the voice of a mother, a daughter, a sister, a friend. Drawing on the experiences of women in the Toronto soundwork industry, this chapter re-situates the value of lived experience in reminding the listener of the human intersubjectivity and empathy involved in listening to the amplified voice. The disembodied woman is a fiction. As Western culture moves toward a changing digital broadcast soundscape, listeners and producers alike need to stop asking which radio voices are preferred and start asking why.

\section{Radio Voice as Extension of Self: Vocal Self-Identity and 'Authentic' Presentation}

Within the modern techno-culture of sound production, the possibilities for manipulation of the amplified voice are readily at hand to change both audience and actor perceptions of the body, identity and connected socio-cultural frameworks of the voice. Yet a long history of radio as primarily an intimate entertainment and information communication system has created an expectation of 'authenticity' from the broadcaster. A prescription of trust taken up by the listener to presume the human being on the other side of the transmission speaking gently into the microphone is presenting their 
'authentic' self. As sound philosopher Schlichter argues, sound technologies of the 20th century significantly impacted our process of listening and consequently changed our "scientific understanding of the body, of perception, and of social and cultural relations. ${ }^{, 25}$ The human voice, in particular here the publicly amplified radio voice as an extension of the body, binds to our understanding of the self and our performative identities from a phenomenological and existential perspective. Chapter Five explores how these expectations and experiences of the embodied vocal self shape the gendered material voice and identities of women in soundwork.

Feminist philosophers, performance artists, historians and media scholars alike have analyzed the ways in which our cultural structures function to both confine women to their objectified passive bodies (visual media) and to estrange them from their bodies (radio) ${ }^{26}$ Through the lens of feminist phenomenology in conversation with the experiences of Toronto's women radio announcers and podcasters, we seek to reconcile the mediated body with the self, to understand how gender identity is experienced in our modern world. Key themes are brought up by these women on the subjects of authenticity, relatability, and conversationality, which constitute both their understanding of the 'real' self extended through their soundwork, while simultaneously harkening back to their conception of the 'ideal' radio voice. This complex relationship between gendered identity production and self-perception furthers desire in our media industry for a de-gendering of the broadcast voice toward a perception weighted on the authentic, relatable and conversational 'real' presentation of the self on the microphone. 


\section{Verging upon a Sonic Multiplicity}

As a radio producer and host as well as a media scholar, the role of the embodied electrified voice - that of other actors and of my own - entices a desire to explore this junction between soundwork and the self. I present a multidisciplinary approach centred on the experiences of individual women to uncover the relationship between gender representation and the material voice, shared industry perceptions of female voice, and how dramaturgical and on-air voice reflect or reject 'authentic' identity and concepts of self. A limitation to my analysis of gendered voice in this work is the lack of experiences brought forward by racialized women within Toronto's radio and podcasting industry. Race, along with gender, sexuality and class impact how each one of us sounds and listens. ${ }^{27}$ The "whiteness" or "color blindness" that continues to be present in our radio and podcasting industry is evident here in the lack of racial diversity among the women who chose to share their gendered experiences of radio voice for academic research. ${ }^{28}$ With this reflection and call to the radio community, I present the individual experiences of the individual radio talent who lend their voice to this work. Although these ten women cannot speak for the entire industry in Toronto, I hope their experiences help move us towards a broader discourse surrounding female and feminine representations in Canadian media.

With an exhibited gender bias of predominately white male voices within North American radio history as outlined by Michele Hilmes in Radio Voices, ${ }^{29}$ the potential for and experience of non-male coded voice within the digital podcast space and future broadcasting becomes an enticing and very real shift in soundwork culture that must be 
explored. The voices we hear on air, through smartphone headphones, or high-end speakers are changing. The monopoly era of the rich booming low frequency male radio announcer voice has gone the way of the floppy disk and the landline phone. As the voices we hear in aural media change, further research into the gendered material voice and the experience of women in soundwork must be done before we can begin to understand the effects that aural representations of women in media have on a larger socio-cultural scale. 


\section{Chapter One}

\section{THEORETICAL FRAMEWORKS}

There is a certain paradox involved in writing visual text about embodied voice and sound. ${ }^{1}$ This contradicting notion, and certainly the historical apprehensions or 'deafness' toward the study of sound and voice across disciplines from media studies to feminist philosophy, has created a gap in academic cultural discourse up until the recent flow of interest in radio and sound studies. Feminist media scholars have historically centered gender and identity on the body and visual texts, with the voice exercised as metaphor - immaterial or interpreted solely as the words spoken. In this vein, the disembodied woman of radio is severed from their physical form. On the subject of gendered voice in soundwork, from radio to podcasting and beyond, I bring forward a feminist phenomenological approach that works to bridge the fields of sound studies and radio studies within a contemporary context to bring embodied experience back to the gendered radio voice. Feminist phenomenology provides a philosophical foundation to approach gendered voice in media as simultaneously embodied and constructed. This approach clears a path to conceptualize the material aspects of the voice amplified and electrified through soundwork - from podcasting to radio - without separating the voice from the cultural and social embodied experience, identity and subjectivity of the self. In this chapter I present a focused construction of the theoretical foundations that influenced my realization of gendered voice within our social and cultural soundscape, and the consequent literature that helped to shape my hypothesis. 
To bring together the intersections of critical feminism and phenomenology, I draw on the works of Don Ihde and Judith Butler. ${ }^{2}$ I then move into a discussion of R. Murray Schafer's The Soundscape to establish the foundations of sound studies in relation to aural analysis and the acoustic environment of radio. The works of Caroline Mitchell, Michelle Hilmes, and Christine Ehrick bring feminist perspective to the field of radio studies with Ehrick's work bridging the gap between gender studies, sound studies and radio studies. ${ }^{3}$ The feminist philosophical theories on voice presented by Adriana Cavarero are key to my understanding of the power of embodied voice while Andrew M. Kimbrough and Anne Karpf provide an accessible historical perspective on voice across disciplines into the twentieth century. ${ }^{4}$ Embodied voice is brought into our modern digital culture, through the work of Madsen and Potts in Voice: Vocal Aesthetics in Digital Arts and Media, a textured collection of articles on voice edited by Norie Neumark, Ross Gibson and Theo van Leeuwen. ${ }^{5}$ I have also included a brief section on scientific and sociolinguistic research of influence to the study of vocal quality and pitch to provide method and foundation to the sonic practice behind the study of voice. To tie these concepts of vocal quality into the modern realm of radio media, I conclude with a short discussion of gendered voice at National Public Radio (NPR) by notable radio scholar Jason Loviglio. ${ }^{6}$ These key texts stand out as foundational to my theoretical framework and interdisciplinary understanding of the sonorous embodied gendered voice in soundwork. 


\section{Critical Listening for Embodied Voice}

Decades of Cartesian thought, of a separation between mind and body, of logocentrism, have created a fracture between the voice and the body. The introduction and proliferation of recording technology widened this chasm to create the disembodied voice of radio and recorded music. Though historically centered on visual experience, perception and the consciousness, ${ }^{7}$ contemporary phenomenology offers a conceptual approach to open our ears toward the auditory dimension. In his groundbreaking study of sound through experience, Listening and Voice: Phenomenologies of Sound, Don Ihde outlines a phenomenological framework for a sound-oriented study of language, voice, music, religion and silence. Originally written in 1976, this comprehensive publication by Ihde offers a detailed theoretical discourse for the study of sound primarily guided by his understanding and expansions on the philosophies of Husserl and Heidegger. Understanding of auditory phenomenology is bounded by "an appreciation for the richness and complexity of experience." ${ }^{8}$ The value of experience is central to any phenomenological approach as, "experience cannot be questioned alone or in isolation but must be understood ultimately in relation to its historical and cultural embeddedness." $" 9$ This centering on the value of experience is integral to the study of gendered voice. The interviews conducted for my study of gendered voice rely on this human value of experience and of presence to give authority to the women who have so graciously given their time and material voice to this research.

In addition, phenomenology of auditory experience provides a framework for critical listening in scholarly research and within our everyday experiences of sound. The 
analysis of gendered voice in soundwork relies on the constant awareness of our own experience of critical listening and our own embodied experience as listener and researcher. Ihde presents the early workings of what can be understood as embodied listening; an approach to listening as a full body experience. ${ }^{10}$ The material voice can also be thought of in this way. As a sonic extension or continuation of the body. A phenomenology of voice in this vein begins by addressing the philosophical question, "what is voice?"11 Here we might understand 'voice' as embodied material voice, a sounding outward that comes to form language based on our human experience of speech even as we develop in the womb. Although Ihde also addresses the use of voice as metaphor and the voices of material things in the world, for the purpose of this study into gendered voice on radio I draw exclusively on his theories of listening and voice in relation to humankind. What phenomenology as conceptualized by Husserl, Heidegger and Ihde does not address is the gendered experience. My work takes up the phenomenological approach to sound and voice and reunites gendered experience with the sonorous body.

\section{Voicing Feminism and Embodiment}

Although Ihde brings forward an embodied phenomenological framework for the study of sound and voice, I require a feminist theoretical approach to ground my examination of gender and identity. A feminist phenomenological analysis of voice must be rooted in both a feminist understanding of the role of voice in gender identity and the phenomenological understanding of lived experience. ${ }^{12}$ Judith Butler's Gender Trouble stands as a foundational text in the study of gender within the modern feminist discipline. 
Butler's work provides a philosophical feminist and linguistic framework to discuss the importance of language and bodily acts in relation to the representations and performativity of gender involved in the continued encoding and decoding of those same acts as gendered. ${ }^{13}$ This model of performativity questions the way in which gender is understood in feminist work. The possibilities brought forward by this model for the subversion of gender norms and the investigation of the constructs of sex and gender are integral to the study of the experience of gender identity and subjectivity across disciplines. Performativity of gender is constituted as a stylized repetition of acts. Simone de Beauvoir asserts "one is not born a woman, but, rather, becomes one."14 Drawing on this sentiment, Butler further theorizes that sexual difference is not a fact but a product of the organizing structures of language and cultural context, an unrelenting question that remains 'unsettled and unresolved. ${ }^{15}$ The participants in my study of gendered radio voice, are radio workers who identity as women, thus it is this constant questioning and the experiences of gendered performativity as outlined by Butler that create the feminist theoretical foundation for my methods of interview and analysis.

Butler's theories - though integral to our understanding of gender identity - lack a solid framework surrounding the voice as an extension of the body and thus a key part of gender performativity above and beyond language. By focusing on the "picture of gender" ${ }^{\prime 16}$ through language and visual performance, Butler simultaneously mutes the significance of voice. Drawing on Derrida, Annette Schlichter theorizes the proximity of voice as the "carrier of self or identity" ${ }^{17}$ which Derrida calls auto-affection. ${ }^{18}$ Here we find a theory for the materiality of the voice, as a condition for the creation of meaning. 
Be it language or the production and reproduction of gendered constructs. The theory of gender performativity emerges as a “critique of 'feminist phonocentrism' (even if Butler herself does not use the term)." 19 Phonocentric logic assumes "a 'natural' relationship of the voice, the female body and female identity as the truth of the woman's self" that predefines a universal understanding of the "woman" identity. ${ }^{20}$ Through Schlichter's work we see a shift in the theorization of voice in Feminist discourse from exclusively metaphorical or simply speech or written word toward voice as sound, as an extension of the body. Butler's gender performativity model can thus be used as a method of counterdiscourse to question the gendered constructs at play in relation to the material voice.

With a shift toward embodied voice as a subject of feminist discourse in recent years, we are experiencing a desire for a more feminist approach to phenomenology on particularly social, political and gender issues. Feminist concerns around materialism or biologism have created certain non-materialist tendencies toward the discussion of voice. ${ }^{21}$ Feminist phenomenologists have taken up this issue of voice as metaphor to shift the symbolic 'voice' toward its vocalic roots. The feminist approach to phenomenology, as outlined by Fisher in "Feminist Phenomenological Voices", deconstructs the gendered voice as both material and cultural. ${ }^{22}$ Fisher brings forward the importance of embodied voice in our understanding of identity but it is important also to note the ways in which human kind can communicate through non-vocal interactions such as sign language and text to voice technology that amplify agency and social power for those who may not have what is considered to be an able-bodied voice. Nevertheless, the embodied voicespeech relationship is a crucial aspect of identity in our Western social and cultural 
structures. The material voice gives us the ability to communicate, to construct and to experience our intersectional and ever transforming identities.

What is lacking in Fisher and Schlichter's approach is the exploration of the significance of amplified voices in media in the perpetuation of these hegemonic gender constructs and perceptions, while simultaneously acknowledging the human embodiment of the presenter. Radio voice is not disembodied. As I explore further in chapter five, through perceptions of radio voice as an extension of self. This embodied radio voice experience is fostered in our digital culture in which the physicality and visual aspects of the announcer can easily be drawn up in a simple google search. Radio is no longer an exclusively aural medium. ${ }^{23}$ The work of Butler and feminist theorists up to this point have brought forward recognition of gender and sex as performative concepts, yet the heteronormative gender systems continue to plague the our social and cultural identities. More work must be done to shed light on the normative and suppressive frameworks, in particular our media industries that perpetuate gender identity constructs through mass media consumption.

\section{Politics of Gender Identity and the Self}

It is impossible to draw on Butler without mentioning Michel Foucault whose work Butler draws heavily on as foundational to the formation of the theory of performativity. The philosophical problem of the subject and of agency - central to Foucault's work - is an important theoretical issue in feminist and post-modern study of gender and the self. The post-modernist death of the subject is a threat to the concepts of 
agency and self-reflexivity that are dependent on there being a subject in work around critical identity politics. ${ }^{24}$ The radio voice embodied sits within a greater network of cultural, social, temporal and gendered experiences that are interpreted and understood based on our own intersubjectivity and self-perception. In The History of Sexuality Volume III: The Care of the Self, Foucault confronts everyday notions of sexuality through a theoretical and practical history of experience and the treatment of sexuality as a 'type of normativity' and as 'a relation to the self'. ${ }^{25}$ Through reflection on his theorization of experience hinged on three axes, ${ }^{26}$ Foucault resituates 'experience' in relation to the third axis 'sexuality' with a focus on "the modality of relation to the self. ${ }^{, 27}$ Foucault's work is revitalized and questioned in contemporary theory toward a critical study of gender identity and power, opening up further exploration into the social and cultural relationship between gendered voice and concepts of self.

For many decades, modern theorists have presented a gender blind notion of the self, but thanks to the work of Judith Butler and Seyla Benhabib, the gendered self has arrived within our broad-spectrum understanding of identity politics in critical feminist and modern communication theory. Benhabib opens Situating The Self with the differentiation between the two Greek notions of time, kronos (chronological time) and kairos (lived time, a moment of indeterminate time in which everything happens, time imbued with meaning) ${ }^{28}$ Like the communication of these dual meanings for time, our understanding of 'the self' has changed drastically since Greek antiquity. Benhabib embraces the notion of embodied self within historical cultural and social context to nullify the illusions of the "disembedded and disembodied subject" found in metaphysical 
traditions of Enlightenment. ${ }^{29}$ Through Butler we can posit there is no fixed identity behind the performativity of gender but that identity is constituted by the very expressions that are said to be its results. Benhabib argues if there is no subject, no self behind these performative repetitions, how can we expect to "pull the curtain down"? The subject is essential to Benhabib to provide agency and selfhood to the vision of feminist politics. Benhabib moves us away from the postmodernist death of the subject toward a notion of selfhood - unwittingly coloured by Butler's theory of performativity that roots our sense of self in relation to kairos. Though Benhabib is true in their acknowledgement of the postmodern death of the subject in Foucault and Butler's work, it is a misstep to presume that presenting gender identity as performative results in the removal of one's agency or subjectivity. Although our identity is not fixed but constituted and repeated through social and cultural norms, we would not have the agency to reflect on or draw on to form a sense of self without temporally stretched and momentary manifestations of subjective selfhood.

The feminist critical notion of 'the self' I present here founded on the work of Butler and Benhabib, are integral to the understanding of embodied voice in relation to not only the gendered self but the experiences and perceptions that emerge within our given social and cultural context. Although Benhabib argues against Butler's model of performativity, the narrative model of identity constitution presented in Benhabib's "Sexual Difference and Collective Identities", pairs well as a reflective means for awakening an understanding around the performative nature of the self through the use of storytelling. ${ }^{31}$ The study of gendered radio voice has a close tie to the use of storytelling. 
As a primarily sound driven and language based media, the study of soundwork voices can further the potential power of storytelling in the construction and awareness of selfidentity. Later feminist works around the notion of 'the self' must continue to draw on the power of storytelling or the recollection of life experience - be it through media creation, interviews and/or dialogue- for the formation of identity as a web or series of selves in perpetual construction. ${ }^{32}$

\section{Self-Perception and The Gendered Experience}

The feminist phenomenological approach to the study of voice in relation to identity has led me to a focus on first person experience, particularly the experiences of the broadcast women whose voice(s) I center this research around. In Joan W. Scott's Evidence of Experience, she argues that although it may be tempting to abandon it altogether "experience is not a word we can do without." ${ }^{, 33}$ The historical use of experience within scholarly works to was to write difference without analyzing how one's experience and thus identity is constructed. Although Scott uses a visually exclusive language of discourse that is deaf to the power of sound, her theories on the importance of a contextualized and structurally aware discourse of experience is utilized within soundworks to create powerful affective story for a public audience through multivoice personal, historical and contextual sonic narrative.

Perceptions of 'the self' as embodied and of the material experiences -which colour our constantly shifting experiences of our own identity - were overshadowed during the Enlightenment only to resurface in feminist/post-modernist and phenomenological discourse. In Primacy of Perception, phenomenologist Merleau-Ponty does not attempt to dissolve Enlightenment notions of rationality or the absolute in his 
articulation of perception. He does however open up the presupposed notion of a universal or absolute perceived world. This opening up of perception provides modern feminist theorists with a foundation toward a multiplicity of perception and a sense of agency and authority in personal experience. The "primacy of perception" as conceived by Merleau-Ponty, is the experience of perception at the moment when "things, truths, values are constituted for us. ${ }^{, 34}$ Our perceptions teach us not to take for granted what is objectively 'real' while summoning us to "the tasks of knowledge and action." ${ }^{.35}$ The notion of time in relation to perception re-emerges in feminist discourse echoed by Benhabib in relation to our gendered understanding of the self simultaneously subjective and objective, as perpetually under construction. ${ }^{36}$ But we must question how we can have a sense of what is 'real' or 'authentic' unless we have some sense of a snapshot of who we are as a subject.

This problematization brings forward the importance of time in our understanding of the authentic. ${ }^{37}$ Echoing Heidegger's term 'Throwness' ${ }^{38}$ the idea being presented here is that we act authentically in the 'here and now'. There is a sense of immediacy to it while also an acknowledgment of how our perception of self is coloured by our past and our expectations of the future. This dual understanding of time as kronos and kairos, is key to understanding how authenticity has resurfaced as an integral element for the radio voice. This is of particular note in relation to 'live' radio as both the presenter and listener are experiencing the voice in the same 'here and now' (give or take a few seconds for reception). Even if the show is played back on demand or re-broadcast, the sound of the voice is re-amplified and experienced by the listener in their own perception of 'here and 
now', of kairos while simultaneously understanding the voice as a voice of the past.

Perception is experienced as individual, but the social, temporal and cultural soundscape that creates a foundation for that perception is founded on both individual and collective experience.

\section{(Re)sounding the Gendered Experience}

My work sits within a growing intersection between the fields of sound studies and radio studies within our digital era. I use the term 'soundworks' put forward by media scholar Michele Hilmes in The New Materiality of Radio, "creative/constructed aural texts that employ the basic sonic elements of speech, music and noise; this excludes the field normally encompassed by the term 'music,' though of course the boundaries are anything but clear. Typically speech is the dominant aspect of soundwork, with music and noise secondary." 39 'Soundwork' becomes a significantly useful term to discuss the current works being produced in radio and podcasting media that potentially underlie the shift in gendered voice in relation to digital access, on-demand, cultural norms and the blurring of traditional radio broadcasting formats.

Before we can begin to attribute terms such as soundwork and soundscape to my contemporary study of gendered voice, we must first trace back the emergence of sound studies as a field of interdisciplinary inquiry. In his groundbreaking work, The Soundscape, Canadian composer and educator R. Murray Schafer advocates for research

into the acoustic ecologies of noise with which we all live within each and every day. ${ }^{40}$ First published in 1977, this book introduced the term "soundscape" as an aural landscape, asking us to tune in and listen more closely to the world around as an expansive musical composition. The soundscape as defined by Schafer is an environment 
of sound, a sonic landscape with emphasis on the way it is perceived by an individual or by society at large. ${ }^{41}$ The term includes tangible environments such as a city or a beach but it can also be an abstract or musical construction such as a radio show or in our modern context, an online or digital environment. Schafer creates a set of terms or features of the soundscape for the sound analyst to utilize when entering the field of study. A system of generic classification into three main themes: keynote, signal, and soundmark. ${ }^{42}$ Here we can see Schafer establishing a set of terms that can be used in practice when collecting sound or "touring" within a given soundscape. These terms have become commonly used among sound studies scholars in recent years across the humanities and social sciences.

Although Schafer wasn't working with the same digital software systems we have to process audio in 2017, it is the discussion of machine listening and sound visualization put forward by Schafer on the importance of sound images for the "preservation and analysis of sound" ${ }^{43}$ that begin my development of aural analysis methodology. Using Schafer's methods, the classification of sound can be conceived through four key domains; acoustics (physical characteristics), psychoacoustics (way in which they are perceived), semiotics and semantics (function and meaning), and aesthetics (emotional or affective qualities). ${ }^{44}$ These key domains or lens for the analysis of sound are crucial to my research. As Schafer puts it, "soundscape studies will be the middle ground between science, society and the arts. ${ }^{, 45}$ My thesis and corresponding radio documentary put this call into action through aural analysis of voice pitch, and data collection of women's experiences of voice in today's Canadian society. Through this process I am able to reflect and listen in more closely to how these recollections and stories intertwine. 


\section{Uncovering the Veiled Histories of Women in Radio}

Radio has been called the 'Cinderella medium' 46 and radio studies has only recently established a space within media and cultural studies. In Women and Radio: Airing Differences, Caroline Mitchell posits that "if radio is Cinderella then women in radio studies still has 'pumpkin' status." ${ }^{, 47}$ But this Cinderella story, the once invisible women of radio has gained considerable ground over the past few decades with publications like Radio Voices by Michele Hilmes, Women and Radio and more recently, Christine Ehrick's Radio and the Gendered Soundscape. Still the radio and podcasting industry continues to see a lack of feminine voices on the air. As feminist radio artist Frances Dyson wrote in Radio Rethink, "The most consistently excluded or derided voice is feminine. ${ }^{" 48}$ Feminist critical and historical research into the area of feminine and women's voices in soundwork - be it radio, podcasting or audio on-demand- must continue to question the lack of representation in sound media and advocate for change.

Hilme's Radio Voices explores the birth of radio broadcasting in the United States from 1922-1952 through the focus on the radio voices, celebrated and censored, which influenced these 'golden' years of radio in its many forms. In chapter five, "The Disembodied Woman”, Hilmes opens with a written account from 1921 of a female reporter-announcer from Memphis Tennessee. ${ }^{49}$ By recording the experiences of Gwen Wagner, Hilmes points to this historical blind spot, a problematic denial of female presence in American broadcast history. Through the words of amateur wireless operator Abbye White, Hilmes discusses the anxieties that women in this field experienced, "Rather fearfully I venture into your contest, for I do not know if we of the fair sex are allowed in or not." ${ }^{50}$ White's anxieties written in her 1923 essay I hypothesize here to be 
echoed still among modern women-identified radio and soundwork professionals. With the question, "Are women undesirable over the air?",51 Hilmes dates the debate over women's opportunities and place on the American airwaves back to a 1924 controversy leading to a virtual disappearance of women's voices in 1930's U.S. broadcasting. Sparked by a letter in Radio Broadcast, Jennie Irene Mix reported that a notable record dealer attributes poor sales of recordings featuring women speakers with a public distaste for female radio announcers on the grounds that "the voice of a woman, when she cannot be seen is very undesirable, and to many, both men and women, displeasing. ${ }^{, 52}$ From Abbye White to the 1924 debate that threatened the radio careers of women in broadcasting, Hilmes focuses in on the biases against womens voices on air. Through Hilmes' identification of some of these major aspects of gender formation in broadcasting, she calls to the academic community to take up an avenue of feminist investigation. My thesis work takes up this call by addressing the gendered experiences of women in the Toronto soundwork industry.

On the topic of gendered radio voice, a key text that provides a collection of cultural accounts and radio women histories is Women and Radio edited by British Radio scholar Caroline Mitchell provides a long overdue collection of works bridging the fields of women's studies, radio studies and broadcast history for future generations of media scholars. This work broadens the dialogue of radio history out of the heteronormative male-dominated past and into a critical movement toward a more diverse radio industry. In relation to the gendered experienced of voice on radio within this collection; "Justifying injustice: broadcasters' accounts of inequality in radio" by Rosalind Gill 
draws on a discourse analysis developed by Potter and Wetherell "to examine broadcaster's accounts for the lack of women DJs at the radio stations where they

work" ${ }^{, 53}$ through the personal accounts of five male DJs and program controllers. Part of the feminist radio studies movement and struck by the lack of British female DJs during her contemporary study, Gill sets out to investigate how the inequalities or lack of balanced gender diversity in radio is made sense of by those within the industry. The qualitative approach taken by Gill not only contributes to the understanding of gender dynamics and inequalities in the radio industry; it also provides a strong approach to discourse analysis of interview material with radio professionals on the subject of radio women.

\section{Radio's Gendered Soundscape}

Gill's analysis brings the value of experience into the larger cultural and social context of gender inequalities in the media industry, but it is difficult not to wonder what the female or feminine radio experience could contribute to the conversation and how those voices in particular, the voices of the women broadcasters, contribute to the soundscape in question. The marriage of gendered voice in dialogue with the term sound studies understanding of soundscape is brought forward in Christine Ehrick's Radio and the Gendered Soundscape: Women and Broadcasting in Argentina and Uruguay, 19301950. This text makes a substantial contribution to the history of women in radio through the rich soundscape of Latin America during the pivotal time period of women's suffrage, WWII and the rise of the Perónist Era. Women in radio, particularly the discussion of women's voices on radio is a subject that still creates heated debate today. Focused on the Rio de la Plata river cultural zone of Buenos Aires, Argentina and Montevideo, Uruguay, 
this study affirms, "the female radio voice introduced a new dissonance into the gendered soundscape. ${ }^{, 54}$ and that the new medium of radio provided women with a platform from which to amplify their voices and be heard at a historic moment of opportunity for the renegotiation of gender roles.

Pulling from sound studies foundations of radio as soundscape Ehrick's research internationalizes the history of women and radio beyond the North American and European lens through a contemporary sound studies perspective. This sonic pathway continually reminds the reader to think of not only the text or speech of female subjects but how their voices work to form conceptions of gender and agency, "tuning in to sound as a signifier of power" within the rioplatense soundscape ${ }^{55}$ The bias against women's voices in 1930's broadcasting and onwards must continue to be critically approached in the academic community. Even as gender roles shift and technology became better adept to the reproduction of higher frequency sound, female and feminine voices continue to be polied on the airwaves. This critical historical approach engages with radio as a sonic technology, posing larger questions about the aural dimensions of gender and modernity that I expand on through my study of gendered voice in the Canadian soundscape.

\section{Conceptualizing Voice in Communications}

The Cartesian separation between mind and body created a fracture between the voice and the body. The introduction and proliferation of recording technology widened this chasm to create the disembodied voice of radio and recorded music. The declaration of the radio voice as disembodied is repeated throughout media and communication 
studies. ${ }^{56}$ Yet to begin to understand the complex cultural and social phenomena within our gendered radio soundcape, the embodied experiences of women in soundwork must be brought into conversation with the amplified electrified voice. Through this work I move to re-centre the amplified and electrified voices of soundwork as the sonorous embodied extensions of the self we experience these voices as in our modern digital culture. Three scholars from three different disciplines are brought together here through the historical study of voice toward an understanding of the embodied voice in communications. American theatre professor Andrew M. Kimbrough traces the theoretical trajectory of dramatic voice studies through the twentieth century, while Italian philosopher Adriana Cavarero draws on a history of philosophy to remind us that "when the human voice vibrates, there is someone in flesh and bone who emits it.",57

Tying together the gendered experience of material sound with the cultural realms of performance and radio production requires a strong foundation of voice studies to contextualize the material voice in our hyper-visual modern era. Translated into English from its original Italian, ${ }^{58}$ Cavarero's For More Than One Voice: Toward a Philosophy of Vocal Expression presents a gendered philosophical treatment on the politics of the human voice. Cavarero presents a vocal ontology of uniqueness in which voice appears as the principal branch that "radically contests the metaphysical tradition that silences the 'I' in flesh and bone." 59 The material voice is central in our understanding of gender and uniqueness through the power of speech in our political and social soundscapes. ${ }^{60}$ Like Schlichter, Cavarero calls out the feminist philosophers of the past on the way in which speech and voice are often construed to be interchangeable rather than symbiotic. Speech 
acts are relational because "beyond the specific content that the words communicate is the acoustic, empirical, material relationality of singular voices." ${ }^{\text {, }}$ There is speech because there is a living, breathing, feeling human from which these words extend through sound in a given space. With an understanding of voice studies within feminist historical phenomenology, we can conclude that whether the material voice comes from a person standing in the room with us or from inside the radio station, over the air and into our stereo speakers, the voice is a powerful tool not only because of its relation to speech but because human voice as sound connects us like nothing else.

A critical approach to the study of voice as a dramaturgical extension of the self furthers the historical contextualization of the gendered voice in relation to performance and media production. Working in a post-For More Than One Voice realm of study, Kimbrough commends Cavarero in his introduction for her contribution to the philosophical study of voice and the auditory, echoing her line of thinking into the history behind our visualphilic culture tracing it back to ancient Greek civilization and the influence of written language on philosophical thought. ${ }^{62}$ This text weaves a crossdiscipline account for the development of renewed interest in voice, language and speech in the twentieth century where we encounter a privileging of the role of electronic communication.

An understanding of the development of vocal anatomy, expression and language in relation to human evolutionary theory and the scientific study of voice is vital to our understanding of gendered radio voice as embodied. Kimbrough notes that in relation to 
spoken languages, "they still acquire meaning deeper than language through our submergence within embodied experience... a primordial dimension that informs our listening to the human voice. ${ }^{, 63}$ We can then associate this primordial human link through voice to the intimacy or connection felt when you hear the human voice on the radio as it isn't simply the presence of a human body but a neurological evolution which has helped to shape this culture of communication in which we live. From here, we move to tracing the auditory dimension and phenomenology of voice brought forward by Don Ihde into conversation with early theorists Sartre, Merleau-Ponty, Husserl and Heidegger ${ }^{64}$ For Kimbrough, phenomenology provides three key concepts that aid in study of vocalized language: "the link of sound with sense, communication as embodied, and the operation of logos in speech. ${ }^{, 65}$ In relation to gendered voice in soundwork, these concepts provide an avenue of exploration into phenomenological and existentialist thought on the voice in communication technology providing a refreshingly contemporary and accessible view on the subject at hand.

Each chapter in Dramatic Theories of Voice presents the voice through a different school of thought ranging from phenomenology to postmodern, but between chapter's five and six, Kimbrough chooses to insert an interlude dedicated exclusively to Walter Benjamin. Drawing on the 1936 essay "The Work of Art in the Age of its Technological Reproduction”, Kimbrough 'teases out' Benjamin's theories of the voice within the soundscape of media technologies. ${ }^{66}$ "The Work of Art" by Benjamin stands a foundational text in the Communication and Cultural studies disciplines through his discussion of the "aura" or uniqueness that is lost through reproduction. Yet, Benjamin 
applauds the sound-synchronized film as a powerful tool of mass information dissemination. Here Kimbrough brings forward a sonic reading of Benjamin's discourse, centralizing on sound - specifically the human voice - as key to completing the illusion of reality, or what I would argue in relation to radio and soundwork the key to the extension of reality. The importance of the four communicative domains toward the hearing of our embodied, sonorous voices brought forward by Kimbrough, "the emotive and instinctual, the mimetic, the linguistic, and the mediated" ${ }^{167}$ echo the four domains in soundscape classification as written by Schafer: acoustics, psychoacoustics, semiotics, and aesthetics. These four domains are echoed throughout my work as well, as I approach the gendered voice as simultaneously material and constructed. This simplified four-part approach provides an important contribution for young scholars and sound studies experts alike to draw on as an accessible guide to some of the most essential ideas on voice and orality across the disciplines.

Of particular note for my thesis work is Anne Karpf's The Human Voice. Echoing Cavarero, Karpf draws on the power of the voice as a 'terrifyingly intimate' gesture of our biological, psychological, cultural and social status. ${ }^{68}$ In doing so, Karpf sets the tone for discussion of the material voice as extension of the body through air and its deeply gendered ties. Drawing on popular scientific study of male and female biological difference in our vocal folds and oral system, Karpf explains how the longer, thinner, and more taut vocal folds of a human female vibrate more frequently to create higher frequency or pitch in sound production. ${ }^{69}$ It is interesting to note, Karpf's attention to the emotive power of the voice in relation to the body. She explains "any alteration to the 
mouth can change the voice, even smiling. It widens the mouth and shortens the vocal tract, smiling can actually be heard."70 Smiling while speaking into the microphone typically results in a more upbeat and positive tone. Even though these biological circumstances are given, cultural and social context can drastically change the frequency, pitch, and overall paralanguage of the human voice.

The most influential outcome of Karpf's work for the study of embodied voice in radio is the discussion of the changing gendered voice in relation to tone and frequency. Karpf argues that women's voices are getting deeper, changing instead of liberating our pitch and tonality from our patriarchal history. While bringing to center the discussion of our changing voices in the Western world, Karpf is careful to note the many geographical and cultural differences in 'ideal' voice and gendered voice norms across the globe. The historical context and differences between Japanese and English women's voice pitch and presentation must be noted. Although my work can be discussed in relation to gender in soundwork and media across the globe, it focuses in on the particular experiences and embodied voices within Toronto's radio soundscape. The pressure for women whether in the radio industry or across the professional spectrum to masculinize their voice is posited by Karpf to be tied to cultural and social concepts of dominance and masculinity. "If men's voices are deep in order to convey the size and mastery essential to triumphing over their rivals, shouldn't we expect the voices of women, as they enter the competitive world in great numbers, to deepen accordingly?"71 Here we see Karpf bring forward an essential indication toward a shifting in the voices we hear in today's digital broadcast realm. Do women in the Toronto radio industry feel the same pressure that Karpf 
indicates in her work? It is certainly the beginning of an in depth exploration into the deepening of women's voices. Karpf also makes the case for a study of the feminization

of the male voice. ${ }^{72}$ Although male voices are not brought into conversation in my work as it is a study on gendered voice from the perspective of women in the Canadian industry- the experiences of feminine and masculine male-identified voices in radio certainly requires further examination from a feminist phenomenological perspective.

\section{Extending the Material Voice in Digital Media}

Kimbrough and Karpf provide a solid foundation for the early theoretical works on voice in twentieth century electronic communication but as we find ourselves immersed in an increasingly fast-paced digital world full of visual and sonic distraction and innovation, we come to question how our voices are reproduced, circulated, reverberated and communicated within our digital media landscape. New media studies approaches have emerged in recent years to speak to the complexities between digital culture and human experience. As podcasting and digital radio become synonymous with traditional radio listening, the digital aspects of radio voice must be addressed. Norie Neumark, Ross Gibson and Theo van Leeuwen present a textured collection of interdisciplinary works exploring the relationship between voice and technology (including radio and podcasting) with a focus on digital culture in Voice: Vocal Aesthetics in Digital Arts and Media. In the introduction "Paradox of Voice", editor Norie Neumark calls for an exploration of the mediated voice as embodied, moving away from the flawed tendency of sound studies to discuss the voice as disembodied when produced with media technology. Neumark draws on the Toronto School, particularly Marshall McLuhan to 
discuss the voice as an extension of 'man', along with the works of Adriana Cavarero and Roland Barthes. In relation to the embodied voice, the discussion of the "grain of the voice" by Roland Barthes in Image, Music, Text and The Grain of the Voice: Interviews 1962-1980, still stands as a cross-disciplinary foundational text in the study of voice and sound. On "the grain of the voice" Barthes famously states:

The grain of the voice is not - or not merely - its timbre; the significance it opens cannot better be defined, indeed, than by the very friction between the music and something else... The 'grain' is the body in the voice as it sings, the hand as it writes, the limb as it performs. ${ }^{73}$

It is this attention to the performing body that brings forward as an evoking of sound embodied. This course of sonic thinking flows throughout contemporary discourse on voice and technology, drawing attention in particular to the history of the disembodied voice in radio studies, "the loss of body in radio seems to be tied to the loss of the sight of the body" ${ }^{\text {"74 }}$ ironically privileging the visual in what is a primarily sound driven field of study.

Contemporary audio media, digital radio and podcasting are central to the amplification and extension of voice in our digital culture. Neumark's discussion of the embodied voice in soundwork is brought centerfold in chapter three "Voice-Cast: The Distribution of the Voice via Podcasting" by Virginia Madsen and John Potts. Drawing from Pierre Schaeffer's term 'acousmatique, ${ }^{75}$ Madsen and Potts argue that podcasting creates a new and revolutionary "extended sphere for the performance of the essentially acousmatic voice" 76 that carries with it traces of the body. Acousmatic/acousmatique is the broadcast and reception of voices within the origin of the sound being visible. Through a history of talk-centric radio, this article creates a short genealogy of the 
podcast as "voice-cast". But the technology used to extend the voice through podcasting creates an argument about the immediate temporality of voice within our understanding of the symbiotic "voice-body", whether the voice recorded and re-amplified can still be considered an embodied one.

This "schizophonia" 77 denotes the splitting of sound from its natural source through technology. Madsen and Potts discuss the way in which the recorded voice in podcasting becomes archived, resulting in a library of voices "suspended" in time, ready for playback. In true McLuhan fashion, this article quickly becomes fixated on the technology as the message rather than how these voices are extensions of the body and unique experiences circulated through sound. Madsen and Potts advocate for the podcast as an empowering listener experience, the ability to subscribe, preview and play on demand. What Madsen and Potts do well is explore the intimate, even erotic potentiality of the podcast for the "grain of the voice" to lead before the content of its message is considered. The spoken voice in audio-on-demand soundwork, in contrast to traditional radio, is often listened to solo through headphones rather than over a set of speakers. Discussion of the qualities of gendered voice is briefly touched on ${ }^{78}$ and Madsen and Potts present a strong argument for the value of the podcast as an embodied voice-driven medium that amplifies the intimacy and grain of the voice. However, in the a-temporal digital world in which we live, does the open circuit of podcasting over traditional radio truly present a more authentic voice or is it simply an issue of soundwork reestablishing its genre(s), similar to attempting to compare reality TV on Netflix to your local television news show. In fact, many podcasts are also radio shows ${ }^{79}$ or feature the same voices that continue to exist in the ephemeral terrestrial radio industry. So what is left 
unclear here is whether the "voice-body" extended through on-demand soundwork are experienced or presented, in relation to the body and the self, differently in modern radio and in podcasting.

\section{Scientific Voice Research of Contemporary Influence}

Within the interdisciplinary realm of voice studies, the influence of modern scientific research and the field of linguistics have made great impact on the ways in which we perceive and come to understand our voices in relation to their historical, social and cultural context. The examination of the relationship between acoustic measure of speech in "Predicting Impressions of Speakers from Voice Quality: Acoustic and Perceptual Measures" by Pittam and Gallois created a scientific foundation for the implications of gendered voice qualities on the social judgement of speakers. Through the analysis of six male and six female speakers recording the same passage, the study found that 'tense' sounding, creaky and nasal voices were perceived negatively in terms of solidarity and status. ${ }^{80}$ It is interesting to note the five voice qualities chosen (breathy, creaky, nasal, tense, and whispery) as they can be easily hypothesized as 'unpleasant' or 'unideal' qualities particularly in a radio broadcast environment. In relation to gender; results showed that 'breathiness' and 'whisperiness' in a masculine voice was perceived to be less attractive than a feminine voice. They found whispery and breathy voices could be tolerated much more than voices found to be particularly creaky, nasal or tense. This analysis by Pittam and Gallois suggests that the 'ideal' voice, at least in Australia, "may contain only small amounts" ${ }^{\wedge 1}$ of these five tested vocal qualities. But as Karpf noted, the perceptions and production of voice can differ greatly from country to country. ${ }^{82}$ The field of linguistics provides a foundation to approach gendered voice on radio as sound 
data but we must be careful to notice the cultural and social factors at play.

Whether it is our gender identity, geographical region, social or cultural contexts transform our perceptions and preferences for the 'ideal' human voice. In the 1988 study "Gender and Culture as determinants of the 'ideal voice"”, researchers Carol Ann Valentine and Banisa Saint Damian surveyed ninety-nine students at the Universidad Autonoma de Guadalajara in Mexico and ninety-eight students at Arizona State University in the United States during the 1982-1983 school year. ${ }^{83}$ Interestingly and opportune for my current research, participants were asked to specifically concentrate on 'the ideal voice of a radio announcer'. Valentine and Saint Damian found that the American overall 'ideal' and 'ideal' male voice shared a close match in descriptions across surveyed participants: both are described as firm, low, cheerful, well-modulated, somewhat slow in rate of delivery, and characterized by clear enunciation. While the female 'ideal' voice type was in contrast, described as delicate, sensual, and soft. ${ }^{84}$ Although this study sought to compare and contrast the 'ideal' voice across gender and cultural difference in Mexico and the United States, their findings in relation to the gendered bias against female voices on air is one for particular consideration as I look to analyze gendered voice in the Toronto radio industry. Based on their findings of gendered ideal voice and the overall 'ideal', they found that a female can either conform to the feminine ideal (soft with a medium pitch) or they can "approximate the 'ideal' speaker's voice by speaking with moderate volume and low pitch; adherence to one ideal type precludes close approximation to the other." ${ }^{, 85}$ Valentine and Saint Damian seem to be eluding toward the same changing gender 'ideals' as Karpf, noting a shift in women's voices toward a deeper more 'masculine' sound. 
The contemporary field of linguistics recognizes the norms, concepts and discussion of 'feminine' and 'masculine' voice as socially constructed phenomena that shift and contract across regions and cultures of our human history. In "Clinical Aspects of Transgender Speech Feminization and Masculinization", Shelagh Davies and Joshua M. Goldberg recognize that voice communication is often decoded within these binary frameworks. ${ }^{86}$ As a speech pathologist, Davies argues that changes to the gendered aspects of voice can "reduce gender dysphoria and facilitate gender presentation consistent with the felt sense of self." ${ }^{\not 7}$ Davies and Goldberg indicate the following; feminine vocal norms include "pitch with a mean $=196-224 \mathrm{~Hz}$ and range $=145 \mathrm{~Hz}-275$ $\mathrm{Hz}$; higher upper and lower limits of range and formant frequencies are higher" while masculine vocal norms include "pitch with a mean $=107-132 \mathrm{~Hz}$, range $=80 \mathrm{~Hz}-165 \mathrm{~Hz}$ and formant frequencies are lower., ${ }^{98}$ It is interesting to note the overlap in pitch range here from $145-165 \mathrm{~Hz}$, as this range could be considered gender ambiguous. ${ }^{89}$ Although Davis and Goldberg add a disclaimer to the article indicating that the existing data regarding vocal gender norms should not be viewed as universally normative, the 'feminine' and 'masculine' data regarding the average range of pitch and indication of formant frequencies are valuable to my study as a foundation for the comparison of sonic data with perceptions of higher and lower gendered radio voices.

These preliminary perceptions of voice quality and societal norms of gendered speech are echoed across the contemporary study of voice in linguistics and psychology. ${ }^{90}$ Through a series of semi-structured interviews with radio employers and educators conducted by Warhurst, McCabe and Madill, the voices on Australian radio are 
shown to have characteristics that make them sound distinctive from the radio voices of the past. ${ }^{91}$ They found that content and personality along with a 'natural' sounding presenter that 'suited the station', were found to be the qualities of a 'good radio voice'. These findings are perceived by the researchers to have potential influence on new models of training and management of radio performers in Australia. Warhurst, McCabe, and Madill open up the conversation of what constitutes a 'good radio voice' and recommendations on how to shift the education model for radio broadcast toward a focus on a more natural voice with 'personality', but similar to Gill's study of the gender in the UK radio industry, ${ }^{92}$ this study lacks the experience of the radio performers themselves on how their voices reflect or reject this notion of 'natural voice'. The lived experiences and perceptions of the women within Toronto's radio soundscape are integral to bring into the conversation here. A mix of audience and industry responses presented for the UK market and Australian market cannot speak to the experience behind the construction of the voices under the microscope.

\section{Toward an Aural Shift in Gendered Radio Voice}

The significance that surrounds my hypothesis of an aural shift in gendered voice within our digital era requires an interdisciplinary approach that ties together the realms of linguistics, phenomenology, feminist sound studies and radio studies. The sonic attributes highlighted by contemporary linguistic and voice study hint toward the need for further historical and cultural research to bring the human experience into conversation with their sonic findings. In his article "Sound Effects: Gender, Voice and the Cultural Work of NPR", radio scholar Jason Loviglio discusses the aural attributes common to the 
female radio voice of $N P R$ being low in pitch, "pronounced lack of pitch variance, a kind of disciplined, flat monotone delivery, with few pitch shifts. ${ }^{.93}$ This feminist conception of the material voices found on National Public Radio (NPR) in the United States traces the shift in the role and sound of women's and men's voices at NPR to the cultural and political movements of the 1960s and 1970s. Through a series of historical mini biographies of influential NPR voices, from Susan Stamberg on All Things Considered to Sarah Vowell of This American Life, Loviglio explores the aural quality of these keynote voices through pitch, intonation, rhythm, loudness, stress and tone. Loviglio provides a refreshing feminist historical discussion of gendered radio voice. Yet this short article lacks the sonic data to back up his claims. It is also important to consider the unique sound of NPR as a national broadcaster and how these voices may different significantly from the commercial radio soundscape across not only America but across the globe.

Although my study focuses on the Toronto soundscape, Loviglio's ongoing work - along with the work of his fellow radio historians ${ }^{94}$ - shows a shift in radio studies toward the incorporation of sound studies philosophy around voice, culture and technology. ${ }^{95}$ Across radio and voice studies, it is clear that women's voices in the Western world have begun to deepen significantly over the last fifty years. ${ }^{96}$ What feminist media scholars bring to the conversation is the beginnings of consideration on the central role that media broadcasting has played in that process. ${ }^{97}$ The feminist study of voice and sound calls for scholars of media studies to cross the interdisciplinary boundaries of history, cultural studies and sociolinguistics to further the study of the mediated human voice. Loviglio presents a strong introduction to the aural shift in 
soundwork in relation to women's voice in broader media through his study of NPR but more must be done to delve into how these varying aurally gendered performances interconnect with the experiences, identities and authenticities of the women behind the microphone.

The lived experiences of women in soundwork are needed if we are to begin to understand the complexities of gendered voice production in relation to the self extended through contemporary media and technology. Phenomenology of listening and voice provides a framework to approach the aural aspects of embodied experience. ${ }^{98}$ Feminist phenomenology furthers this philosophical foundation to approach gendered voice in media as simultaneously embodied and constructed. ${ }^{99}$ The influential work on gender and women coming out of radio and sound studies, in conversation with these equally earoriented philosophies, has begun to clear a new path for the contemplation of lived embodied experience extended through our media. The field of sociolinguistics provides a foundation of findings grounded in mixed method approach for the study of voice in our given social and cultural context, ${ }^{100}$ while the study of voice breaks new ground in the modern field of digital cultural studies to contextualize the sonorous embodied voice extended through our digital world. ${ }^{101}$

The study of gendered voice in broadcast media bridges these many schools of thought to try and understand the complex experiences and perceptions of gender representation in our mediated world. In relation to lived experience and feminist conceptions of identity, this subject has yet to be fully explored within our contemporary Canadian soundscape. My research along with the gendered voices of Toronto's radio 
soundscape can begin to move toward the conceptualization of material aspects of the voice amplified and electrified through soundwork - from podcasting to radio - without separating the voice from the cultural and social embodied experience, identity and subjectivity of the self. The integration of pitch and frequency methods used in sociolinguistics is brought into dialogue with feminist phenomenology and the contemporary study of radio and sound within our digital world to begin to understand and question the structure of the patriarchal broadcast soundscape. With a theoretical framework and foundation of interdisciplinary literature from feminist phenomenology to digital voice and media studies, I move to outline the qualitative research methods and data analysis used for my exploratory treatment of the gendered voice in soundwork within the localized Canadian media soundscape of the Greater Toronto Area. 


\section{Chapter Two}

\section{VOICING CRITICAL FEMINIST METHODS}

A 2015 study released by the Global Media Monitoring Project (GMMP), shows that over the ten years studied (1995-2015) women's share as reporters within the global media industry has dropped on radio and television by four percentage points. ${ }^{1}$ As earoriented scholars we must ask, 'what role does the female or feminine voice play in the continued exclusion of women-identified speakers within our broadcast soundscape?' The human voice holds clues to our social, political and cultural histories. Like a fingerprint or snowflake, the female or feminine radio voice must be analyzed as a unique extension of the speakers' identity within Canada's male-dominated media industry. Through a multidisciplinary approach centred on the experiences of individual women in radio and podcasting soundwork, this study works to uncover within these primarily sound-driven media; the relationship between gender representation and the material voice, shared industry perceptions of female voice, and how dramaturgical and on-air voice reflects or rejects 'authentic' identity and concepts of self. My research methodologies are semi-structured interviews with radio broadcast and podcast talent, as well as aural discourse analysis in conversation with frequency/pitch analysis of select interview materials through a grounded phenomenological feminist framework.

In addition to this thesis, an accompanying audio documentary features broadcast interview material with select woman-identified soundwork hosts and personalities. This documentary serves as an artistic yet 'authentic' representation of these women's 
experiences of their own voice and the surrounding historical circumstances leading up to our current changing media landscape in relation to the voices we hear through our headphones and car radios. As Jonathan Sterne points out in Being 'In the True' of Sound Studies, "One of the main methodological problems for people who do interdisciplinary research is that different disciplines speak of the same phenomenon as if it were two or more totally different things." ${ }^{2}$ Through the methods outlined here, I explore the intersection of voice as material sound - an extension of the body and thus individual visceral identities - and the weight of the women's voice as collectively political and historically coded.

\section{Samples: Toronto Women in Soundwork}

Between October and December 2016, I conducted interviews with ten participants. This is a non-random purposive sample of a select population of women in Toronto's soundwork industry. There are approximately ten thousand Canadians working

in the radio broadcast sector. ${ }^{3}$ Yet there are currently no published numbers in relation to women working on air, let alone the Toronto market so while the sample is small it is a reflection of feasibility and accessibility. The sample size of ten does however reflect the modest ten percent slice of the broadcasting revenues radio continues to hold here Canada. ${ }^{4}$ Much like the small percentage of revenue share radio holds here in Canada, women's voices are underrepresented in our broadcast soundscape. The interview subjects of this thesis are women-identified peoples who currently work as host or talent in music and talk-based radio and/or podcasting formats. Subjects interviewed were restricted to those with a minimum of two-years' experience as professional 'voice talent' 
ideally with diverse backgrounds. Interview subjects were acquired through personal connections in the broadcast industry along with additional research into the current soundwork talent pool based in the Greater Toronto Area.

The participants in my study are neither news exclusive presenters nor primarily voice actors in fiction-based soundwork. All the radio and podcasting women here are first and foremost music announcers or show hosts in talk format soundwork. Though select participants do voice work across many media formats from commercials and fiction work to 'top 40' radio, the presentation format of music announcing and talk based soundwork are the foundation for the responses given. The radio industry in Canada continues to be a predominantly white-dominated field, ${ }^{5}$ which is reflected in the participants who chose to come forward to publicly share their experiences. ${ }^{6} \mathrm{I}$ hope that through further discussion of gendered voice, we can not only bring more women, feminine and gender queer voices to soundwork but expand the conversation to further incorporate race, sexuality, cultural difference, vocal disabilities, accents and more into our Canadian broadcast soundscape. No one person can speak for an entire group or industry, but it is my hope that through these ten women we can begin to understand the gendered experience of soundwork in relation to the embodied voice.

A single semi-structured interview was conducted with each of the subjects to be used for analysis and in the creation of the radio documentary. To randomize potential participants and mitigate any potential personal selection conflicts, a snowball technique was employed by including in my interview protocol a question to each subject 
interviewed to pass along my contact information to one new potential candidate to interview for this research. After all the interviews were complete, audio recordings were transcribed and the analysis began with NVivo 11 data analysis software while the aural analysis and treatment of recordings were prepared in Pro Tools audio production software and Praat speech analysis software. I am grateful to all my participants for their dedicated time and unparalleled contribution to this work.

\section{Interviews in Ear-Oriented Research}

To construct a valuable discussion around the topic of women's voice in soundwork, the voices and experiences of these women must be brought to the forefront. The semi-structured interview is a method of research with which I was fairly comfortable, but under a much more stylistic mediated guise for the purpose of radio, documentary or entertainment fare. Each interview had a run time of 20-45 minutes and was conducted by the primary researcher to provide adequate time to explore points brought up by the interviewee. The experiences brought forward through this interview series provide the foundation for my analysis. As Merleau-Ponty wrote, "it is through

experience that we have the idea of being." $" 7$ The experiences -individual and collectiveprovide clues to the broader conceptions of women's voices on air from both the listener and professional broadcaster perspective. I have chosen a semi-structure protocol format so that I can include well-researched and structured questions while creating a more conversational interview through expansion on interesting points brought up by the interviewee during the process rather than having to stick to a rigid question and answer format. 
The semi-structured interview format provides my research with a framework to discuss the gendered experience and material voice in a more conversational form, giving the participants an opportunity to dwell on concepts of voice as sound and to recall stories of their professional experience. In Doing Interviews, Kvale notes that, "the qualitative interview is a key venue for exploring the ways in which subjects experience and understand their world." ${ }^{\prime 8}$ As discussed in 'Chapter One', experience is precious in the positioning of the self as subjective and intersubjective while acknowledging the performativity of gender identity. Who else can offer a better understanding of the experience of gendered women's voice in soundwork than the women of soundwork themselves? The self-perceptions that arise from this process are invaluable. The individual interview in semi-structure form- person to person - allows these experiences to organically surface. It is clear that interviews are an ideal method to capture and analyse the experiences of both individuals and, once analysed, the collective themes that emerge.

A problematization of working with human subjects, though through a qualitative communicative approach, is the discussion of inequality within my study as it may involve participants who could find themselves in highly vulnerable circumstances because of the social and/or legal stigmatization that is associated with their activity or identity if disclosed within my research. ${ }^{9}$ Privacy and confidentiality in the distribution and publication of my work must be acknowledged. As I have conducted semi-structured interviews for my thesis and for the accompanying audio documentary project, a consent form was drawn up and approved by the Ryerson University Ethics board to address 
privacy and confidentiality. ${ }^{10}$ Although an option of anonymity was given, all subjects were confident in their experiences and chose to have their identities made available for publication and use in the radio documentary without masking or alteration.

\section{Pitch and Frequency Analysis: Voice as Sound}

To compare perceptions of voice brought forward in the experiences of my interview subjects with the broader perception of gendered material voice across disciplines the pitch, frequency and timbre of the sound of the voice can be analysed, interpreted and coded. As discussed in Analysing Sounds by Christoph Maeder, sound is key to communication and our individual as well as collective sonic experience. ${ }^{11}$ The materiality of the embodied voice through what some refer to as paralanguage ${ }^{12}$ brings the sonorous voice itself into the conversation alongside the spoken experiences of Toronto's soundwork women. The fields of speech psychology and linguistics reveal the value of such a combination. Demanding more attention to be brought to bear on this area of study, contemporary research by Jeffery Pittam and Cynthia Gallois draws on this dual analysis of perception of voice quality in relation to acoustic measurement. ${ }^{13}$ Their dual analysis provides a valuable reference point for my own study. In contrast, I have chosen to have the subjects indicate their own judgment of voice rather than drawing on outside judges for perception. In addition to these judgements, I ask how they think listeners perceive their voice. The radio documentary which will be made publicly available for reference to these voices also works to open the dialogue outward, inviting comparing and contrasting views from the broader public on the subject on the experience of gendered amplified voice for future use. 
The practical aspects of recording professional quality sound for such an analysis can include challenging factors such as wind, echoes, reverberations and unplanned for sources of noise like fans or fridges. Professional recording devices and equipment along with years of practiced skill in the operation of said equipment as a media producer are of great benefit in the documentation, production and collection process. The material voice must be recorded with minimal background noise and interference to avoid issues with playback and interpretation in the transcription, coding and analysis stage. Similar analysis of the recorded voice in selected interview materials takes place to listen for differences and trends in the sonic qualities of voice among interview recordings. This type of aural analysis opens up the possibilities of discussing the material voice in relation to socio-cultural constructions of gender and the relationship between identity performativity and gender representation in media.

Professional equipment is integral to the proper capture of audio for analysis and documentary use. Recording equipment used during the interview process include the Zoom $\mathrm{H} 4 \mathrm{~N}$ portable recorder on which I am trained and quite comfortable as well as a variety of lavalier and handheld microphones depending on the location for the interview to take place. The use of proper broadcast equipment is a priority but minimal instruction was required to train the interviews subjects on proper microphone technique or voice presentation. As radio and podcasting talent, they are all quite experienced in front of the microphone. The broadcast interviews are recorded in a high quality digital .wav format at a sample rate of $16 \mathrm{bit} / 44.1 \mathrm{khz}$ for consistency in post-production. During the interviews, the Zoom $\mathrm{H} 4 \mathrm{~N}$ device is placed on the table or surface closest to the subject 
unless the interview space is loud or particularly noisy in which case a close microphone was chosen to suit the environment and interviewee comfort for a conversational environment during this style of longer form research interview.

\section{Discourse Analysis: Voice as Speech}

While an aural approach to the analysis of my interview material offers insight into sonic trends, shifts and differences in our gendered radio soundscape, the value of discourse analysis cannot be ignored. While this work centres on the embodied voice, the responses and stories conveyed through voice as speech - and through the use of language - provide further material to uncover patterns and broader themes of gendered experience of radio voice. The relationship between language and voice are crucial to the encoding and decoding of meaning. Within an ethnomethodology and interview analysis as primarily conducted within my project, the functional or action orientation of discourse becomes the focus. The discourse in question for my project is the collection of interview materials and their corresponding oral-to-text transcriptions. Radio researcher Rosalind Gill outlines four main themes for discourse analysis: "a concern with discourse itself; a view of language as constructive and constructed; an emphasis upon discourse as a form of action; and a conviction in the rhetorical organization of discourse." ${ }^{.14}$ These four points provide an entry to discourse analysis with a supposition that responses given are a clear form of action and expression for these women. Responses can be gut reaction, well thought out answers, speculation or theoretical conjectures, regardless they all provide evidence toward a greater discussion of the gendered broadcast soundscape. 
Through a discourse analysis of interview and transcribed audio; the use of rhetorical, communicative and corresponding language based techniques can be analysed in conversation with aural analysis of the same materials to provide a rich array of material for interpretation and construction. The second theme brought forward by Gill, "a view of language as constructive and constructed," 15 becomes crucial to the study of voice in relation to gender and identity. If we are to discuss women's voices, we must always be aware of the performativity and ongoing construction of gender and thus identity. ${ }^{16}$ Aside from the performative nature of identity, similar experiences and phenomena can be described and expressed in very different ways by each individual within a given community. In our awareness and critical approach to gender and identity -within its historically corresponding social, cultural and political ecologies - we must then analyse language in relation to voice with the same understanding and insistent questioning. Although Gill argues that "discourse analysts do not regard texts as vehicles to find out about some reality assumed to lie beyond or behind language," ${ }^{\prime 17}$ this method of analysis in conversation with aural analysis can create a robust multi-method approach to discourse interpretation beyond such a text exclusive reading while still ensuring the value of verbatim speech-to-text for qualitative research. Although the transcription of interviews is a time consuming data collection technique, it is a key aspect in the analysis of embodied radio voice.

Interestingly, during their discussion of coding and analysing discourse Gill draws on her analysis of interviews "with broadcasters involved examining the accounts they gave for the lack of women working in radio." ${ }^{\prime 18}$ Gill's reflection on her own coding and 
analysis process brings forward a strong reference to draw on as I researched and conducted my own discourse analysis. A crucial note that comes out of Gill's article is the duty of the discourse analyst to not only examine the way in which language is used, but also "what is not said - the silences." " Although the inclusion of silence could be potentially difficult to code, it becomes elemental when vocalized hesitation, variance in breath or affective pause can be incorporated in the software assisted aural analysis and documentary use of the same source material.

\section{Grounded Theory and Analysis Software}

Another approach to my interview material is through the qualitative inquiry offered by grounded theory method. Grounded theory refers to this both the method and the product through the conceptual analysis of data collected which in this case, are a series of interviews with Toronto soundwork women. Grounded theory emphasizes studying processes in the field, engaging in concurrent data collection and analysis, the use of comparative methods, and insisting upon the elaboration and reflection of tentative categories as they are created. ${ }^{20}$ The grounded theory approach that fits well within my phenomenological and feminist frameworks is a constructivist approach. The constructivist "challenges positivist elements that ignore reflexivity, overlook ethical issues, disregard issues of representation, and do not attend to researchers' agency in constructing and interpreting data. ${ }^{21} \mathrm{~A}$ reflexive approach to the collection and analysis of my interview data helps resolve my interdisciplinary inclinations, which takes into consideration both the affective emotional and logical interpretations of interview materials. 
Grounded theory coding involves the fundamental action and reaction process of taking data apart and defining how the codes and themes are constituted. ${ }^{22}$ I have chosen to use Computer Assisted Qualitative Data Analysis Software (CAQDAS) to help manage and analyse my interview data in the contemporary digital workspace. Although CAQDAS can be a useful instrument to aid in the management and grounded theoretical analysis of my interview data, I must make sure to continuously question the given parameters and how the software interface may influence my thesis construction and overall findings. Through exploration of NVivo for qualitative research, the software has shown itself to be a useful tool for both the management and analysis of transcriptions for discourse analysis. It is important to stay somewhere between a suspicious and an empathic interpretation when utilizing CAQDAS, ${ }^{23}$ continuously staying conscious of swaying one way or the other during the coding process.

NVivo 11 software not only makes available text for coding but audio files as well. This function proved to be inessential for audio data analysis. Pro Tools 11 audio production software along with the well-established freeware Praat used by linguists for analyzing speech were used to analyze voice. Developed by Paul Doersma and David Weenink of the University of Amersterdam, Praat is open source software with a range of useful functions for the analysis of speech from pitch tracking to measuring harmonic amplitude and frequency across the given spectrum. ${ }^{24}$ Pitch measurement in Pratt of my audio recordings is brought into comparison with related themes in the discourse analysis that arise in discussion of gendered radio voice. Pro Tools is used for visual reference, clip edit and playback. Although my analysis through Praat is primarily pitch based, the ability to playback and visualize the interviews in Pro Tools presents a secondary way to 
organize and interpret interviews with a dual sensory approach.

As discussed in my chapter on theory and literature, linguistic research into voice and speech communication has brought forward a contemporary groundwork for the analysis of gendered voice within the performative constructs by which we live our daily lives. The study by Australian researchers Warhurst, McCabe, and Madill on what makes a good radio voice provides a strong example of the use of semi-structured interviews for the study of radio voice, ${ }^{25}$ while Pittam and Gallois offer an example of acoustic measure for the analysis of voice quality and perception. ${ }^{26}$ These two studies in conversation with the range of pitch data sets of Davies and Goldberg provide a foundation for the analysis of paralanguage considered 'feminine' and 'masculine' within our contemporary social and cultural context. ${ }^{27}$ Although these data sets could be considered outdated -ten years since publication - they give us a starting point for comparison of the given perceptions of material voice with the quantifiable data we can derive from the embodied human voice as sonic information.

\section{Radio Documentary as Aural Research Approach}

Although the creation of my documentary is a secondary and consequent analysis of the interview materials and voices of my participants, radio documentary as research creation has the ability to ground the awareness of the researcher. The documentary is meant to showcase the 'sound' of the material voices themselves to re-voice the quotes and experiences shared here as text. As a study emphasizing the importance of voice in our experience of gender and media, it is important to have these voices presented as both speech and sound. 
The process reminds me that I am an embodied listener within the gendered radio soundscape of my participants. Sound theorist Walter Gershon found that:

The ability to listen to multiple contexts tapped into the sonic imagination in terms of questions of representation, the ability for the audience to have an embodied, aural experience with participants' voices, ideas, and soundscapes in ways that conveyed an affectively rich sense of place. ${ }^{28}$

This sonic approach to interview material offers a context for its use as not only documentation for audio-to-text transcription, but to create new ways to engage with and present qualitative research that re-centres the voices of experience. Sound becomes undeniably methodologically valuable as it lays at the most basic level of our human existence, "utterly individualistic and inescapably sociocultural in their interpretation."29 The process of radio documentary as a secondary practice-based research method works to add an alternative contrasting and potentially comparative analysis to traditional written qualitative analysis.

\section{Concluding Reflections}

The voices we hear on air, through smartphone headphones or your car stereo, are changing. The era of the booming masculine radio crooner has past but what voices will take their place? As the voices we hear in aural media change, further research into the gendered material voice and the experience of women in soundwork must be done before we can begin to understand the effects that aural representations of women in media have on a larger social and cultural scale. Whether it is the analysis of dialogue in media or qualitative interview analyses of real-world conversations the goals of both are the same, 
to discover "the background expectancies and codes that lie behind everyday behaviour. ${ }^{30}$ But what of the soundwork host who through experience and story plays a key role in the understanding of female representation simultaneously within soundwork, within the broader conversation of our current media climate and within the real-world. It is through this multidisciplinary approach of semi-structured interviews, discourse analysis, and analysis of pitch, along with an audio documentary, that my research works to vocalize the experiences of individual women in radio and soundwork. The findings of this study through the outlined methods in this section lay the methodological foundation to unearth the relationships between gender representations and performativity, identity perception and the intimacy of the amplified material voice. 


\section{Chapter Three}

\section{THE 'IDEAL' RADIO VOICE:}

\section{Pitch and Perception of Gendered Vocal Performativity}

Pitch, loudness, and tempo - some of the features that make up paralanguage - can be more important than language itself. For paralanguage doesn't just support words but gives them life.

- Anne Karpf, The Human Voice

With an abundance of choice in what we listen to, what we choose to add to our mediated soundscape, perceptions of low/deep/masculine voice as preferred or 'ideal' for soundwork are beginning to become a standard of the past. Across the globe from Japan to Sweden to Canada, the gender norms and expectations of the 'ideal' voice are a direct reflection of that society's political and cultural framework. In The Human Voice radio scholar Anne Karpf asks the question, why has the female voice deepened over the last fifty years? ${ }^{1}$ Karpf's question is re-contextualized here a decade later through the exploration of pitch and perception within the Toronto broadcast soundscape. Are women's voices in our Canadian gendered soundscape continuing to deepen toward a 'masculine' or gender ambiguous presentation? Although our perceptions of what is deemed to be an 'ideal' radio voice are changing along with increased access to a multiplicity of voices through podcasting, women's voices in public space continue to be policed, subject to higher scrutiny and judgment than their male counterparts. One must contemplate whether it is the higher pitch and spectral flux of the woman's voice that stirs such debate throughout our broadcast history or is the 'feminine' voice materialized simply part of a larger political soundscape of gender inequity and patriarchal power. 
The gendered voice in our contemporary Canadian soundscape is explored here with a focus on the relationship between pitch and perception. Auditory differentiation of gendered voice is a complex neurological process within the auditory cortex of the human brain, ${ }^{2}$ but when considered in dialogue with the concept of gender performativity, we must approach these perceptions as construction through repetition within our social and cultural histories. In the context of this interdisciplinary query, I bring pitch analysis into conversation with the perceptions of gendered radio voice as experienced by the women who add their own voices to this gendered soundscape. Traits of the contemporary 'ideal' radio voice lead us toward an 'ungendering' of vocal performativity while perceptions of select women's own voices on air tilt us back toward the gendered biases of the past. Considerations of the 'least ideal' or undesired' radio voice offers a glimpse toward a more conversational and popularization of a more 'feminine' sound on the microphone while pitch analysis of select participants show a gender ambiguity colored by our gendered historical preconceptions. Echoing the complexities of gendered performance in our current political and social climate, the perceptions of voice I draw on related to pitch, frequency, and gendered performativity, are the personal experiences of women working in the Toronto radio and podcasting industry.

\section{What is the 'Ideal' Radio Voice?}

"I used to think for a woman's voice on radio it had to be deep and authoritative, and booming. More masculine sounding and I used to think that if you were going to get a job in radio you had to have that certain prototype female voice, basically a man's voice or as close to (laughs)." - Dani Stover, Toronto Radio Host 
A key inquiry and underlying theme of my interview series is to question the notion of the 'ideal' radio voice as masculine. It is widely understood that the deep masculine radio voice was (and some would argue still is) the standard to which all other voices are held. In the 2013 study on what makes a good radio voice, conducted by Warhurst, McCabe and Madill, ${ }^{3}$ the Australian trio found that on the topic of gendered radio voice, select participants in the study indicated "that men wanted to hear men and women wanted to hear men on air..." and "on commercial radio the proportion of men to women is still hugely weighted towards male voices."4 During my interview series, participants were asked a similar question, "What is the 'ideal' radio voice?"

Interestingly, what may be considered gendered responses were not the most popular or common traits given. The top four traits found through discourse analysis of the ten interviews were 'Authentic', 'Conversational', 'Relatable', and 'Clear'.

\section{'Ideal' Radio Voice Traits}

\section{Authentic}

Conversational

Relatable

Clear

Table 3.1 Top 4 Traits of the 'Ideal' Radio Voice

Not only are these top traits gender ambiguous, they are also subjective sonic traits that can be considered unrelated to pitch and any obvious or clear material analysis. How does one go about sounding "authentic", "conversational", "relatable" and "clear"? The answer is anything but crystal. Podcaster and writer, Julia De Laurentiis Johnson perceived the 'ideal' voice as rooted in personality rather than vocal pitch or material form; "the personality in their voice insinuates a sense of authenticity that they haven't been told how to sound, it's welcoming to my ears. It sounds like I'm in a conversation 
with them." ${ }^{, 5}$ The days of the radio announcer voice are fading away, and the rise of the 'authentic, 'regular everyday person like you and me' has arrived. Johnson hints toward a desire for human connection over any sort of preference in sonic tone or presentation. Radio veteran, Stacey Englehart, puts it simply, "there is no ideal radio voice. When I started it was just 'trying to sound an announcer' and now that's just out the window... People want a real person." ${ }^{\prime 6}$ Yet the question still lingers, indeed we may be more receptive in our citizen journalist-podcasting era to listen to a multiplicity of voices whether they are trained announcers or not, but we must consider which voices are rising to the top. Which voices come to represent the professional public and commercial Great Toronto Area broadcast soundscape and in turn shape our perceptions of gender and identity in the media.

\section{A Voice Among the Voices: Perceptions of One's Own Voice}

"I think it's just a voice among the voices" - Kat Callaghan, Radio Announcer

As we experience the perceptions of low/deep/masculine voice as 'ideal' vanish from the discourse around radio voice, it is startling to note that when the women of soundwork are asked about their own voices, half of respondents indicated a perception of their own voice as 'lower' or 'less feminine' than the 'average' woman's voice. Comments including "in terms of how my voice sounds, I probably have a deep voice" and "a bit lower than usual it's not super feminine" ${ }^{8}$ were widespread among participants. Digital Radio Host Eleanor Grace indicated, "I definitely have a low register voice for a female identified person." ${ }^{\prime 9}$ As one of the youngest participants in this study, it is of particular interest to note the lingering historical influence of the lower register voice as 
the voice that is found to be most suitable for broadcast. Grace also indicated that she was often told as a teenager and into her twenties that she had a voice for radio. She expressed a lack of understanding around why her voice in particular was found to be a 'radio voice', "I'm not really sure what that means because I just have this voice." ${ }^{10}$ But the observation can still be drawn that Grace's lower voice is part of the sonic puzzle that makes her voice suited for radio. Her voice sounds to others like the voices that have come before her to pave the aural perceptions within our society of what makes a voice suited for the air. Grace is not the only participant to indicate a perception of their voice as 'lower' or 'deeper' than the 'average' woman's voice while also indicating encouragement to enter the broadcasting field because they have a voice for radio. This indication of experiencing comment on their voice as a radio voice, in all cases was tied to a lack of full understanding as to why their voice in particular was a voice for radio. As shown in the table below, difficulty describing one's voice is just as common as the perception of their voice as lower than 'usual'.

\section{Trait/Perception of Own Voice \\ Indicated Difficulty Describing \\ Average/Normal \\ Lower Than 'Usual' \\ Fast Talker \\ Conversational}

Table 3.2 Top 5 Traits of Participants' Own Radio Voice

While many participants had an ongoing awareness and foundational desire to understand their voice in auditory terms, half of the participants also indicated a difficulty in describing their voice. One participant indicated "I'm having trouble finding good describing words for it, ${ }^{11}$ also indicated spending time reflecting and working on their 
voice every day to improve their overall sound. Here we are again reminded of the enormous difficulties in describing the voice. Unlike the human ability to describe our visual appearance, the lack of common language around voice, a crucial extension of the body so intimate to our presentation of self, is startling. Media Theorist Gunther Kress famously stated the (material) voice as signifier carries meaning that cannot, for formal and ideological reason, be translated into the written medium. ${ }^{12}$ The human voice still carries with it, an inexpressible quality of human connection and material experience that seems to surpass any confidence in adhering to it such concrete terms as we do so easily with our visual markers.

While half of participants indicated a perception of their voice as lower or deeper, half also indicated a sense of their voice as average. This perception of their voice on radio as average or normal as well as the indication of conversationality among participants, are much more congruent with their conception of the 'ideal' radio voice as 'authentic' and 'relatable'. ${ }^{13}$ The dichotomy of perception here shows a lingering of bias toward higher pitch voices or 'feminine' voices on air while indicating a shift toward a more ungendered focus on the personality and 'authentic' presentation of the voice. Potentially the most startling trait that saw trend among half of participants was the indication of their voice as 'fast'. Select participants signified that they must consciously remind themselves to slow down. This conscious indication of preference to a slower vocal presentation is consistent with the trait of 'clarity', the clear voice as ideal for radio. 
Moving back to the outlying theme of lower or less feminine perceptions of voice, the field of linguistics offers an influential intersection into the vocal gender 'norms' of 'feminine', gender-ambiguous and 'masculine' voices. Harkening back to our earlier discussion of the intersection between the scientific study of voice and the construction and repetition of gender norms through performativity, the perception of one's own voice as 'lower' or 'less feminine' is brought into conversation with the pitch range found in the analysis of five participants voice recordings.

\section{The Matter of Vocal Pitch and Perception}

"It's just my voice now, but a lower version than what I was used to." - Stacey Englehart, Radio Host and Voice Over Artist

Five participants from the interviews conducted were chosen for pitch analysis based on the quality of the recording and the five participants' uniformity in employment as professional radio announcers. While all participants work in professional soundwork from radio to podcasting, the focus of this chapter is what constitutes the 'ideal' radio voice, thus the five participants who participated in an in-person interview and work within a traditional radio format (public and commercial) were chosen as most suitable for comparison between pitch and perception of voice. Thirty seconds of each interview file containing consistent vocal activity were played back through Praat analysis software to measure the median, mean and range of pitch for each voice. Findings in pitch analysis versus perceptions of voice as high or deep, masculine or feminine are based on the vocal pitch gender 'norms' - produced for the New York Speech \& Voice Lab ${ }^{14}$ - and based on data from five cisgender and transfeminine linguistic studies. ${ }^{15}$ In the study of pitch related to gender recognition and 'feminine' voice, we must be careful not to make 
universal absolutes about the following data. The chart provided by the New York Speech \& Voice Lab is based on normative pitch data found within the North American English-language field of linguistic study, and as this thesis research is based in feminist and queer phenomenological philosophies, correlations drawn between pitch and perception are approached with the understanding of binary gender as an ongoing social and cultural construction.

As discussed in the earlier section titled "A Voice Among the Voices", half of the participants indicated a perception of their voice as lower or 'less feminine' than the 'average' woman's voice. Interestingly, these perceptions of their voice as lower are consistent with pitch analysis across the board. Speech researchers Davies and Goldberg present Feminine vocal norms for pitch with a Mean of 196-224 Hz and a range $=145$ $\mathrm{Hz}-275 \mathrm{~Hz} .{ }^{16}$ Compared to their data, the five participants (as shown in the chart below) have a median and a Mean pitch that fall below the mean pitch of 'feminine' vocal norm while still falling within the range of auditory recognition of their voice as 'feminine' or 'female'.

$\begin{array}{lrrrrr} & \begin{array}{l}\text { median } \\ \text { pitch } \\ (\mathrm{Hz})\end{array} & \begin{array}{l}\text { mean } \\ \text { pitch } \\ (\mathrm{Hz})\end{array} & \begin{array}{l}\text { min. } \\ \text { pitch } \\ (\mathrm{Hz})\end{array} & \begin{array}{l}\text { max } \\ \text { pitch } \\ (\mathrm{Hz})\end{array} \\ \text { Kat Callaghan } & 181 & 191 & 71 & 450 \\ \text { Dani Stover } & 166 & 187 & 75 & 450 \\ \text { Siobhan Woodrow } & 163 & 174 & 66 & 496 \\ \text { Stacey Thompson } & 182 & 187 & 76 & 440 \\ \text { Susan McReynolds } & 182 & 179 & 70 & 322 \\ \text { Combined Average } & 174.8 & 183.6 & 71.6 & 431.6\end{array}$

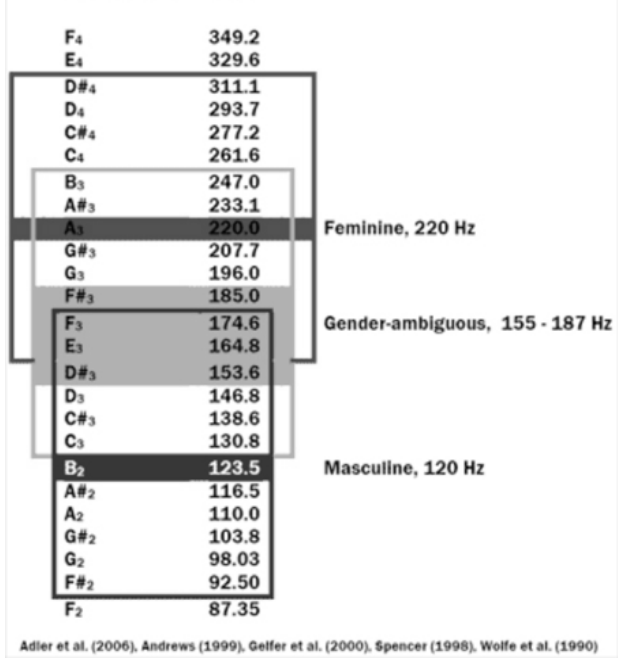


When compared with the pitch chart created by the New York Speech \& Voice Lab, ${ }^{17}$ which includes a gender-ambiguous range of pitch, participants consistently fall within the lower 'feminine' range and more intriguingly within the average range considered to be gender ambiguous. Although pitch is but one of many factors involved in the auditory recognition of gendered voice, the consistency here of lower range pitch among all five woman-identified radio announcers shows that the radio industry, or at least the managers and programmers hiring on air talent, are still perpetuating the long standing inclination toward 'lower' or 'masculine' voices as the radio 'ideal'. This consistency in lower range female voice could also be related to the rate of voice training and broadcast education indicated by participants. Seven of the ten interviewees indicated some level of post-secondary education in radio broadcast while five indicated a form of professional voice training for broadcast presentation. Stacey Englehart, who has worked in the radio industry for over twenty years, indicated a perception that her voice was too high when she began in radio broadcast, "I needed to lower it so I practiced by really lowering my voice to give me a mid range." 18 Dani Stover, a radio host in the alternative rock format echoed Englehart's emphasis on the importance of vocal practice stemming from a foundation set in broadcast school, "we would do voice exercises and doing different reports and documentaries. We had a teacher, Evelyn Macco, who would do voice exercises with us, teach us how to sit up properly and she had one of the best deep authoritative voice, one of the best." ${ }^{\prime 19}$ The role of broadcast education in the repetition and dissemination of the lower and practiced voice 'ideal' is consistent with the 2007 study of radio announcing students done by Borrego, Gasparinin and Behlau. ${ }^{20}$ Their 
perceptual analysis of twenty-five students in Sao Paulo Brazil found that the posttraining speech samples were considered "the best productions in $80 \%$ of the evaluations." 21

Training of the voice for radio and media presentation brings us back to the perception of the 'ideal' voice as 'authentic' and 'conversational' yet 'clear' and arguably 'lower' or gender-ambiguous in relation to the normative or average North American female voice as we have found in our pitch analysis and perception of one's own voice among participants. So far we have discussed perceptions of the 'ideal' radio voice in relation to perceptions of one's own gendered voice on air, how gendered perceptions have become secondary to the 'conversational and 'authentic' sound. To understand how these perceptions of radio voice are changing in our current digital era, we must ask what voices are now considered 'undesired', or 'obsolete' within our media soundscape.

\section{Most 'Undesired' Radio Voice}

"I think I was raised on the Peter Mansbridge voice so I feel very relaxed
around that voice because I was told it was a trustworthy voice. However,
when I do hear those voices now I feel that feeling and at the [same time], I
feel annoyed that it's just another person that sounds like that. I think the
voice that used to calm me is the voice I'd like to hear less of." - De
Laurentiis Johnson, Podcaster Writer

To fully grasp the trajectory of the 'ideal' radio voice, we must also come to understand what is now considered to be the most undesired. As we have seen through our analysis of the 'ideal', we are moving towards an 'ungendering' of the 'ideal' voice form one of masculine or deep sonic traits to one of conversational and relatable presentation. But as we have found in the trend toward a deeper female voice on air through the study of pitch and as Anne Karpf notes in her groundbreaking work The 
Human Voice, women's voices on air are certainly changing but they seem to be changing toward a more masculine or deeper sound. ${ }^{22}$ If we are to truly experience a sense of gender ambiguity and multiplicity among our mediated voices in Canada, voices, which fall outside of these norms, must also find their way onto the airwaves. As we will see in the responses of my participants, while the deep booming male voice is no longer 'ideal', the high pitched or amateur voice is still considered 'undesired' as well.

The number one trait that participants indicated as 'undesired' for broadcast is the phony or inauthentic voice. Five out of ten participants indicated distaste for a voice that sounds phony, as if they are putting it on. While certain participant's indicated these voices seem to be fading away, others indicated, "it's still out there. We used to call them Ronny Radio voices. ${ }^{, 23}$ This 'Ronny’ personality is certainly one that I can draw up in my mind. The 'Ronny' radio announcer is the over the top, over-excited sales person style of Top 40 radio announcer, typically a mid-range rather than low pitch male voice. There was a sense among the soundwork women that radio's strength as a medium is in its intimacy, "where you are supposed to be the closest to your listeners. We're not television stars or actors, were telling you what's happening in your community."24 Regardless of whether you are discussing radio in the terrestrial traditional sense or in the audio-on-demand podcast, this sense of intimacy and thus sense of 'authenticity' or sincerity is a foundation for the 'ideal' radio voice. 


\section{Undesired Radio Voice Trait}

\section{Putting it On (Phony/Inauthentic)}

High Pitched

Loud, Authoritative or Booming Male Voice

Untrained/Amateur

Table 3.5 Most 'Undesired' Radio Voice

While the undesired trait of 'putting it on' or unauthentic' voice bodes well for the gender-ambiguous traits of the 'ideal' indicated in this chapter, the indication of the high pitched voice as 'undesired' by three participants brings us back to our historical propensities for the low or lower pitch voice. "There's nothing more annoying than a high pitched voice on air" one participant expressed, "it's just a very sexist thing but its radio, people want a more soothing voice, when it's too high pitched, too annoying people tune out." ${ }^{25}$ While on the other end of the frequency chart, we find that three participants indicate distaste for the booming male voice. As we have seen in the past as outlined in Gill's 1993 study “Justifying Injustice”, women's voices in the radio industry often come under fire as being too 'shrill' too 'dusky' and just plain 'wrong', leaving her tempted to suggest "that the only way a woman can succeed is by sounding like a man." ${ }^{26}$ This negation of the high-pitched voice as suitable for the media realm of radio seems to continue in our social and cultural soundscape today.

Inversely, we are seeing a push back against the other extreme, being the overtly masculine or deep sounding voice on our Toronto airwaves. From these findings it is tempting to consider that rather than moving toward a multiplicity of voices or an opening up of pitch range from high to low regardless of gender, we seem to be experiencing a 'normalization' of voices taking place. Although we may be becoming 
more accustomed to different accents or personalities on air - at least here in Toronto the 'ideal' radio voice may be shifting toward a sphere of gender-ambiguity outside the realm of our traditional binary gender norms. Whether this shift toward a consistently lower female or midrange male voice will expand to include a wider range of sonorous voices and an opening of the airwaves across vocalities only time will tell.

\section{Conclusions and Reflections}

Unlike the Greek myth of Echo - the nymph punished by Juno with the loss of her own material voice - the ideal radio voice today is one that does not simply repeat the words of another but presents an 'authentic' presentation of their true self, their own ideas conveyed with a relatable, clear and conversational tone regardless of gender. With an abundance of choice in what we listen to, what we choose to add to our mediated soundscape, perceptions of low/deep/masculine voice as preferred or 'ideal' for soundwork are beginning to become a standard of the past. Our perceptions of what is considered to be the 'ideal' radio voice are certainly changing but whether the voices we hear are changing towards a liberation of voices regardless of pitch, gender and sociocultural context are still up for debate.

The higher pitch or 'spectral flux' of the woman's voice continues to be hit with rapid-fire scrutiny while the deep booming male voice becomes a part of Toronto's radio soundscape history, an undesired sound for the future of broadcast. As Ihde notes in Listening and Voice, "experience cannot be questioned alone or in isolation but must be understood ultimately in relation to its historical and cultural embeddedness." 27 The 
experiences and perceptions drawn upon here are exclusive to the Great Toronto Area and are only a few of the many women bringing their voice to the soundwork industry. What we come to understand through these perceptions of the 'ideal' radio voice in relation to performativity in our particular social and cultural moment is that the modern feminist movement toward equity and equality can be heard echoed in the ideals of women in soundwork. We can see the influence of Butler's theories at play in relation to the construct of gender through voice and the repetitions of 'feminine' and 'masculine' vocalities. The awareness of identity as "performatively constituted by the very expressions that are said to be its results" ${ }^{\prime 28}$ can be heard in the desire for a more 'authentic' style of performance over the overtly 'phony' Ronny radio voice. The historical biases experienced by women toward the 'feminine' or higher pitched voice on air continues today yet in a much different form. As we have found through the analysis of pitch and perceptions of gendered vocal performativity through the eyes and ears of Toronto soundwork women, we are moving toward a more equitable broadcast soundscape. But the move toward a multiplicity of voices, as we will explore further in the next chapter, is a slow uphill battle within our patriarchal past and capitalist present. 


\section{Chapter Four}

\section{GENDERED EXPERIENCE OF SOUNDWORK:}

Feminine and Female Voices in Sound Media

\section{"Are Women's Voice Still Undesirable - Over The Air?"}

Perception of female voice in radio echoes through a vast and complex history of bias and scrutiny. As a result, the 'ideal' radio voice has long stood at a troubling gendered impasse. In Radio Voices, Hilmes outlines three historical events of the 1920's and 30's, including a heated public debate in American broadcast that led to the segregation of the women's voice to daytime hours in North America and the privileging of the 'deep' or 'masculine' voice for the primetime radio announcer. ${ }^{1}$ From then on and into the 1990's and early 2000's, booming masculine voices continued to dominate the American airwaves. Meanwhile across the border, the Canadian radio listener would turn the dial to hear the authoritatively deep yet approachable CBC voice of a Peter Gzowski and Peter Mansbridge, or the American 'shock jock' stylings of their local or syndicated Top 40 AM broadcast. Through the work of radio scholars and historians such as Michele Hilmes, ${ }^{2}$ Caroline Mitchell ${ }^{3}$ and Christine Ehrick, ${ }^{4}$ we see a rewriting of broadcast history taking place to include the pioneering voices of women in radio over the past 100 years from Europe to South America. As Mitchell states in her introduction to Women and Radio, “loudly voiced arguments about women's voices being unsuitable for radio and disliked by listeners have now been more or less smashed." ${ }^{, 5}$ Yet as we have seen in our previous chapter on the 'ideal' voice, unambiguously 'feminine' and high-pitched voices continue to be perceived as 'undesired' for broadcast, while lower pitch or medium pitch voices dominate the airwaves. It is the personal lived experiences of the 
women and voice talents targeted by these biases and perceptions that can situate our understanding of how amplified voices are shaped through historically gendered and performative work.

In this chapter, the gendered experiences of women in soundwork highlight the continued policing and high level of judgment when it comes to women's voices amplified. With a focus on the Toronto soundscape, experiences of discrimination and negative comment towards their own voices are compared with discrimination or bias experienced by participants toward the voices of other women. It is through the repetition of language, gendered stereotypes, radio tropes and other performative acts that gender inequity persists within a masculine/feminine power dynamic of our own construction. ${ }^{6}$ As Walter Ong wrote in Orality and Literacy, the power attached to the spoken word amplified is intertwined with "the fact that oral peoples commonly and in all likelihood universally consider words to have magical potency, at least unconsciously, with their sense of the word as necessarily spoken, sounded, and hence power-driven."7 Power is a central theme in the discussion of gender politics within our modern soundscape. Fifty years prior to Cavarero's work on the subject, ${ }^{8}$ sociologist Hannah Arendt in The Human Condition writes "speech is what makes man a political being." 9 Thrusting us back into the sounding body, the contemporary feminist phenomenological approach compels us to note that speech is not voice for the voice is sound and "speech is its essential destination."10 The amplified radio voice extends from the body as sound and carries with it our unique vocality from which gender, age, culture, emotion, and experience are decoded and received by the listener. 
The embodiment and vocalic nature of the voice precede notions of 'feminine' and 'masculine' within our complex history of bias that perpetuate gender norms and performative repetition in our cultural and social moment. Power plays an important role in the experiences of women in soundwork, as power is tied directly to the amplified voice. Power not only lies in the amplified voice but in the experiences themselves, the evidence of experience. ${ }^{11}$ Who is deemed to have the authority or 'ideal' voice for amplification through soundwork is still an ongoing struggle for the professional radio broadcaster. The gendered prejudice and fears of the radio industry brought to light by Rosalind Gill over twenty years ago still permeate our broadcast soundscape. ${ }^{12}$ When we turn on the radio, 'masculine', male or deeper voices continue to outnumber 'feminine' or female perceived voices in Toronto. The professionalism and determination of radio trailblazers such as Marilyn Denis, Erin Davis, and Maureen Holloway have paved the way for women on the Toronto airwaves but as the experiences of today's radio and soundwork women show there is still much work to be done.

\section{"She's so shouty": Experiencing Bias' toward Women's Voice Pt. I}

"You tend to get the same things time and time again. Oh she has a really screechy voice or a squeaky voice or high pitched voice or what was it thrown at Hilary Clinton... she's so shouty." - Susan McReynolds, Radio Producer and Host

Post-feminist rhetoric claiming the triumph of equality along with the push for fundamental rights during the first and second wave feminist movement have recently come under fire as intersectional and third wave feminists gain increasing momentum in

our Trumpian global political landscape. ${ }^{13}$ In the radio and podcasting realm, the post- 
feminist dream is far from reality. On the topic of inequity toward women's broadcast voices, participants in the sample for this research were asked, "Do you recall ever hearing any negative comments about other women's voices on air?" All participants during the semi-structured interview series indicated that they have experienced negative comment or discrimination toward women's voices on the microphone. "Yes, all the time," one participant said without hesitation, adding that "people make comments about everybody but I think women specifically can sometimes still have it harder." ${ }^{14}$ The overwhelming response toward having experienced or heard negative comment toward particularly 'feminine' or female voices was presented as a given facet of working in the broadcast medium. These gendered voices, based on their 'feminine' or female sonic attributes, continue to be policed within this soundscape.

Select participants reflected on the sonic characteristics or traits that come under fire when women experience negative comment toward their voice. 'Uptalk', 'vocal fry', and higher pitched voices received particular note as traits discriminated against related to the female or 'feminine' voice on air. These characteristics share a common thread of historical association toward the younger less educated 'feminine' voice typically linked to the "valley girl" or blonde bimbo" stereotype. American films like the cult classic "Clueless" can be attributed to the stereotypes perpetuation and parody of the 'uptalk' and 'baby talk' style of speech. ${ }^{15}$ One participant remarked, "Yeah I think the thing I hear the most is her voice is so high she sounds like an airhead. The pitch of your voice has no correlation with your intelligence." 16 'Vocal fry' and 'up talk' have gained considerable 
attention not only in mass media and journalism ${ }^{17}$ but in the academic research community as well.

Garnering the 2016 publication of Dr. Paul Warren's comprehensive work Uptalk: The Phenomenon of Rising Intonation. ${ }^{18}$ The myth around the speech trait as exclusive to young women is argued against by researchers such as Thomas J. Linneman through the study of gendered intonation variation, ${ }^{19}$ while a related study in the field of speech and language communication by Joseph C. Tyler indicates a continued perception of 'uptalk' as linked to the young 'valley girl' stereotype with participants noting the contemporary influence of pop culture socialites the Kardashians. ${ }^{20}$ Similar to the study of pitch, the phenomena of uptalk vary by region and cultural context. Linguists Liberman and Warren suggest that Canadians have a unique shape to their uptalk, ${ }^{21}$ while a now 28 year old study monitoring Toronto radio stations discusses instances of uptalk in use among young females. ${ }^{22}$ We can take from this finding to theorize that uptalk is not a generation specific or globally uniform phenomenon. Yet Western society continues to identify this style of speech with a lack of education and a lack of power simultaneously connected with the feminine voice. The particular attention brought to the scientific and linguistic study of uptalk in relation to young women; works only to perpetuate the biases against women with up talk phonation and their consequent connections to the valley girl stereotype. Further scholarly work is needed around the connection of up talk outside of binary gendered comparison and focus on young English speaking women to rid future public opinion of these biases and negative comment on 'feminine' uptalkers. 
In contrast to the valley girl high pitch impression of 'uptalk', 'vocal fry' is found to be naturally associated with low frequency pitch production, ${ }^{23}$ which may explain the increased use of 'vocal fry' by women as an attempt to modify their voices to fit the patriarchal soundscape. Vocal fry is a distinct creaky sounding phonation, or glotteralization, produced by a patterned low frequency agitation of the vocal cords when the speaker comes into their lower vocal register. ${ }^{24}$ Although 'vocal fry' is tied to low pitch production and thus would be a logical product of the more 'ideal' radio voice in relation to low or medium pitch voices, for the female or 'feminine' voice the perception of 'vocal fry' has been found to have the opposite effect. In the influential 2014 study of vocal fry in relation to young women's success (or lack thereof) in the American labor market, researchers Anderson, Klofstad, Mayew and Venkatachalam, found that young adult female voices with 'vocal fry' phonation are perceived as "less competent, less educated, less trustworthy, less attractive, and less hirable", recommending that young American females steer clear of 'vocal fry' if they wish to maximize their opportunities in today's labor market. ${ }^{25}$ The rise of 'vocal fry' among English speaking young females may be just another way in which our voices are changing generation to generation but as Ira Glass, host of NPR "This American Life" pointed out recently - men do it too. ${ }^{26} \mathrm{He}$ admitted to his own voice often including the much-despised creaky phonation, but while his female staff members receive hate mail about their vocal fry Glass as a maleidentified radio voice seems to be immune to the phenomenon. While the women of NPR's This American Life receive negative comments for their creaky speak, one of the younger participants in my study indicated they don't mind vocal fry at all. "I kind of like it," she stated, "I think I'm just so used to it - men and women - I think it's generational 
more than gendered sometimes..."27 This comment stood out as an indication that although we are experiencing discrimination against the 'vocal fry' of younger female voices in our soundscape, these perceptions may simply be cultural and social growing pains as new generations enter the labour market and take over more dominant roles of power and influence.

While some participants noted sonic characteristics of the feminine' or female voice as the target of bias, others noted a link to the broader issue of gender discrimination as the true foundation for experienced bias against women's voice. "It's not necessarily the tone or inflection of their voice, it's just because they're female," one participant remarked while adding, "for some people it's hard to hear females talking the way females actually talk." ${ }^{28}$ Sounding too 'feminine' or 'girly' in both presentation and the content being communicated can become a nagging anxiety to overcome for women entering the on air radio/soundwork field. This type of gender inequality is normalized through the continual repetition of stereotype related to the 'feminine' voice and the continued domination of our soundscape by more masculine coded presentation. As Ehrick notes in Radio and The Gendered Soundscape, "If the female voice broaches these boundaries, it disrupts the sonic environment and the result is often perceived as dissonant and jarring." 29 Our ears as both producers and listeners become accustomed to this patriarchal reverberation.

The shared experience of sexist remarks is an undertone to many participant responses, particularly in how a woman's voice is deciphered by the listener into a 
preconception about their visual appearance. "I had a friend who got insulted because someone thought they'd have bigger boobs" shared an interviewee, "How does that communicate, how do you sound like you're busty?? ${ }^{30}$ This leap in judgment based on the sound of a persons voice harkens back to our discussion of the "valley girl" stereotype, or the perception of the female radio host as an unauthoritative and/or sexualized voice in the radio soundscape. The primarily auditory exclusivities of the medium provide an ability to excite the imagination of the listener - which can be an empowering experience - but this power over imagination is not an isolated transmission. The listener, regardless of how the announcer perceives to be presenting their voice, relies on their personal experiences of visual and auditory memory to make their interpretation and judgment of the person on the other side of their radio broadcast. The dominant perceptions of and bias toward 'feminine' and women's voices we continue to experience today are rooted in our long history of patriarchal power. The results of these inequalities and power dynamics mean women's voices are listened to in a vastly different way than one listens to the male or masculine voice. Until we begin to hear a multiplicity of voices on in our broadcast landscape, we can only expect history to repeat itself.

\section{"I'm so lucky": Experiencing Bias' toward Women's Voice II}

"I think thankfully that isn't something that I've personally seen. I've been lucky in that regard." - Elanor Grace, Digital Radio Host

While all study participants unanimously indicated experiencing some sort of discrimination or negative comment toward women's voices on air, the responses given when asked if they had experienced bias toward their own voice are varied and much 
more complicated. While three participants expressed that they had experienced discrimination or negative comment, four participants indicated that they have not experienced any bias toward their own voice or could not recall an experience.

Interestingly, all three participants that indicated they had never personally experienced bias also indicated feeling 'lucky' or fortunate to not have had this experience, demonstrating that the experience of discrimination against the 'feminine' or female voice is perceived as common within our Toronto soundscape. The expression of feeling humbled by or fortunate for the success of receiving a professional on air host or announcer role is not uncommon among female presenters. ${ }^{31}$ But the overwhelmingly ubiquitous response of 'I'm so lucky' to have not experienced discrimination against my voice and consequently their principle tool for continued employment in the Toronto soundwork community may surprise others in what could be perceived as a progressive and highly educated society.

Among the women who indicated having experienced bias toward their own voice on air, vocal volume or loudness arose as a key factor in their experiences of gendered voice. It has long been assumed that women's voices are naturally less powerful than the male voice. ${ }^{32}$ As Anne McKay recalls in Women and Radio, these gendered notions permeate our public, private and scholarly environments. ${ }^{33}$ Toronto School theorist, Walter Ong's hypothesis that amplification technologies such as the microphone and broadcast technologies such as the radio helped to remedy this "natural deficiency in the female voice" 34 demonstrates the presumption that women's voices were (and still are) often experienced as 'deficient' and 'quiet' making the amplification technology a tool to project the female voice into public space. Women's voices amplified outside the realm 
of the private -through the medium of radio in particular - have a long history of controversy. The intimacy and aural focus of radio mixed with the gendered vocal performativity associated with the 'feminine' proved to be startling for listeners of early radio. ${ }^{35}$ The volume and power to take up space in the public sphere continues to be a disquieting issue in relation to gendered voice as it continues to reverberate through our contemporary soundscape. One participant noted from their experience, "if there's ever been complaints it's more like just 'shhh' a little bit." ${ }^{36}$ A response such as this could be explained away as a response to the distorting effect that over-modulation or vocal loudness in decibels $(\mathrm{dB})$ can have on the studio microphone yet numerous participants throughout the interview series repeat this policing of the volume of a women's voice as a central issue in the gendered vocal experience. One participant recalled the critique of Hillary Clinton as "too shouty" 37 while another recalled being told that she shouldn't talk too quickly or too much, a policing of her vocal volume and authority to take up space that continues to influence her vocal presentation into adulthood. ${ }^{38}$

While the ubiquity of experiences of negative comment toward other womens voices on air found in this study indicate a continued historical perception of biases, the mixed responses given by radio and soundwork women on their personal experience of policing or negative comment toward their own voice indicate a positive shift toward a more equitable gendered soundscape in the Toronto market. Another indication of a positive shift in our soundscape is the infrequency or rarity of these comments as noted by participants who shared their personal experiences or the experiences of others. Although participants indicated a potential shift away from gendered discrimination in soundwork, the increased awareness or contact with negative comment through social 
media must be taken up as a critical factor in how we experience discrimination in our digital world. When asked about the frequency of negative comments, one participant remarked, "thankfully not often but every now and then you might receive something negative on social media. ${ }^{39}$ The influence of Twitter, Instagram and Facebook on the circulation of negative comments toward women and the proliferation of online trolling such as the \#Gamergate controversy in 2014 - is certainly a well-known subject in our digital culture. ${ }^{40}$ However, the current and future implications of social media on the soundwork industry as a screen medium stand as a relatively new topic of inquiry that requires further investigation. ${ }^{41}$

\section{Gendered Radio Tropes and The Patriarchal Ear}

While the experience of discrimination and negative comment toward women's voices on air resulted in a divided response between personal and public, a key subject that arose out of this inquiry is the use of heteronormative stereotypes and gendered tropes on air. With a focus on commercial radio, four participants indicated the continued use of gendered stereotypes and gender-defined positions in the broadcast industry. One participant noted a historical lineage of putting women into designated 'female' or 'feminine' radio roles:

I do think there is a space in radio where women fit in historically where a woman can very easily become the laugh track or do the entertainment hits, the co-host voice. I think that might not be a bias in a bad way, it gives women an opportunity to work but certain stations rest on those laurels... and that's where there is a bias... I don't know if it's always intentional but radio is very much a boys club. ${ }^{42}$

Perceptions of gendered topics on radio such as 'entertainment hits' can be traced back to the creation of women's programming, which began to surface on commercial 
stations as early as the 1920 's. As noted by Hilmes, the growing number of radios emerging across America in each and every home made the medium a logical choice for women's programming. ${ }^{43}$ Radio as a new medium enjoying rapid growth in listenership during its early years, played a key role in the formation of 'feminine' stereotypes and women oriented subjects from entertainment news and gossip to fashion and handy tips for the modern housewife. Although such subjects continue to have widespread appeal, the gendered division of what is considered 'feminine' lowbrow media versus the more 'masculine' highbrow media subjects of politics, arts and culture projects an archaic division of binary gender norms. ${ }^{44}$ These divisions perpetuate the perception of woman's voice as one lacking authority while simultaneously discouraging male-identifying citizens from engaging in what may be considered 'feminine' subjects out of fear of losing their status or authority in our social and cultural patriarchal society.

The gendered soundscape of radio is not only perpetuated through the mediated division of 'feminine' and 'masculine' topics of discussion but temporally through the inequities of primetime versus daytime programming. Unlike the realm of television which relegates primetime to the evening hours - terrestrial radio hits its peak listenership during the early hours of the commuter drive. Morning shows are the bread and butter of radio station programming. Influenced by American broadcasters such as Mary Margaret McBride and Pat Weaver, mornings shows had sprung up on radio stations all over North America. ${ }^{45}$ This 'new' show format began to follow a similar formula station-to-station, showcasing real-life or fictional husband and wife teams, growing to be referred to as "Mr. and Mrs. Breakfast" programs. ${ }^{46}$ In discussion of discrimination against women's 
voices, one participant brought up the gendered notion of the morning show format. She shared the observation, "there are no two women mornings shows" ${ }^{\text {"47 }}$ in the Toronto commercial radio market. Then recalled having a conversation around the subject with a male colleague:

Certainly women are commercial and you can make money off a morning show like that. His answer [male radio employee] was 'people flip through the dial so quickly they think that two women will sound too similar, won't have two distinct voices. It won't make sense and it will sound like one person talking to themselves and where's the fun in that'. I kinda thought that it was ridiculous. Women sound like all sorts of things and any voice is like a fingerprint or a snowflake and any voice sounds different from another one. I understand passive listening maybe somebody isn't putting in a lot of effort to draw that distinction between two voices but surely there's two men in a lot of morning shows as well, so why not make that same comparison. ${ }^{48}$

Based on Numeris data from the time period of my interview with Woodrow, six morning shows of the top ten most listened to radio stations (AM/FM) in the Toronto area were hosted by men solo or as a duo and the other four stations have a male \& female duo. ${ }^{49}$ Going back to the history of the "Mr. and Mrs. Breakfast" formula that became a radio staple in the 1940 's, it seems that we continue to see this particular style of morning show succeed along with a surprising number of male voices in a "Mr. Breakfast" formula dominating the radio morning soundscape. Morning shows often feature host banter interspersed with news, interviews and commentary, and in the case of a music station, the talk is interspersed between the programs set musical selections. While many of these Toronto stations feature a male lead, there while often times be a news or entertainment woman while certain stations feature all-male or male heavy morning show teams. ${ }^{50}$ It is important to note that some Toronto stations do indeed have female morning shows hosts ${ }^{51}$ but the prevalence of male voices over female ones on the top ten stations in Toronto 
speaks directly to the patriarchal ear hinted at in Woodrow's interaction with her male colleague.

These findings echo the broader conversation in gender studies around women's work and which roles in the workplace are perceived as female or feminine. In Canada, women began increasing their presence in the labour market at a steady pace from the 1950 's up until the 1990's, moving from the home into the workplace. ${ }^{52}$ Yet the "working woman" in today's society is still perceived as tied to the traditionally female domestic sphere. ${ }^{53}$ Cooking, cleaning, caring for children, making clothes, organizing social gatherings or entertaining in the home are invaluable life skills regardless of gender yet the inability to separate what is still perceived as women's work from the woman in the workplace, can be linked to the inequalities and barriers that women continue to face within our modern economic system. ${ }^{54}$ From the experiences of Toronto radio women, it seems as though these cultural notions of women's work continue to influence managerial and programming decisions around topics and roles for female talent on the mic. These issues of gendered stereotypes and inequality in radio become intertwined with the oppression or lack of voices across pitch, gender perception, class, race, and sexuality perceived as 'undesired' or 'unfitting of the format' by the patriarchal ear. Until radio stations stop perceiving women's voices in prime drive hours as taking a 'risk', ${ }^{55}$ 'feminine' voices will continue to be relegated to the afternoon timeslot or the secondary roles of entertainment news and traffic reporter.

\section{Toward a Polyphony of Gendered Experience}


The act of listening is polyphonic. ${ }^{56}$ Whether we are focusing in on our local news report or the listening to the latest $\mathrm{CBC}$ podcast, our ears are always open to the world taking in not only the voices around us but also our own voice toward a constant polyphony of simultaneous external and internal aural experience. As we have seen through the repeated themes and connections between these individual broadcast women, our experiences are always already "intersubjective. ${ }^{, 57}$ If all we ever hear are the same voices, a singular language echoed station to station, that becomes our understanding of voice. Women's voices remain undesirable on air only to the extent that the broadcast industry and our society as a whole continue to echo and repeat the same voices that our patriarchal ears have grown accustom to through reverberating performativities. A long history of discrimination against 'feminine' and female voices haunts our contemporary soundscape, particularly when it comes to gendered voices amplified. 'Uptalk', 'vocal fry', and higher pitched voices continue to receive particular discrimination directly tied to the 'feminine' gendered voice on air because they are perceived as outside of the radio 'ideal'. It is no coincidence these vocal traits share a common thread of historical association toward the younger less educated 'feminine' voice typically linked to the "valley girl" or blonde bimbo" stereotype. A hyper feminized stereotype used to take away any authority from the girl, the woman, the feminine, the voices perceived to be outside of or uncharacteristic of the heteromasculine voice of patriarchal power.

The gendered radio soundscape cannot anticipate change until we make change audible. Drawing on the experiences of women in the Toronto soundwork industry, this chapter re-situates the value of lived experience in reminding the listener of the human 
intersubjectivity and empathy involved in listening to the amplified voice. The disembodied woman is a fiction. Every voice you hear is the voice of a mother, a daughter, a sister, a friend. The amplified radio voice extends from the body as sound and carries with it our unique vocality from which gender, age, culture, emotion and experience are decoded and received by the listener. ${ }^{58}$ As Western culture moves toward a changing broadcast soundscape, listeners and producers alike ought to stop asking which voices are preferred and start asking why. Our sense of self is intimately tied to our voices and the heard voices of others. Consideration of the vocal body allows us to question the relation of the "biological and the cultural, the somatic and the symbolic" in the assembly of bodies and subjectivities. ${ }^{59} \mathrm{~A}$ greater understanding and appreciation for the uniqueness of the material and speech-language voice as an intimate extension of the gendered self can open the airwaves towards a multiplicity of voices. 


\section{Chapter Five}

\section{RADIO VOICE AS EXTENSION OF SELF:}

\section{Vocal Self-Identity and 'Authentic' Presentation}

The ear distinguishes the sound of the voice and knows it to be human not only because it vibrates in the specifically human element of speech, but also because the ear perceives its uniqueness - Adriana Cavarero, For More Than One Voice, 178.

When you present your voice to the public, you open yourself out toward each and every listener. The sound of your voice carries with it traces of age, sex, gender, sexuality, culture and many more facets of your collective and individual identities. Decades of Cartesian thought - of a separation between mind and body - of logocentrism and visual-philic tendencies have created a fracture between our understanding of this unity between the voice and the self. The introduction and proliferation of recording technology in the nineteenth century widened this chasm to create the perception of the disembodied radio voice. ${ }^{1}$ However, as Cavarero reminds us "when the human voice vibrates, there is someone in flesh and bone who emits it." ${ }^{, 2}$ Fascinated with this concept of the self and the limits or manipulability of self-identity through vocal presentation, American performance artist Sarah Jones prefers to call what she does with her voice “characterizations of people."3 In her 2009 Ted Talk showcased on NPR's podcast TED

Radio Hour ${ }^{4}$ Jones stuns the audience by switching seamlessly between her welleducated sounding theatrical American ('real') voice and her eclectic variety of characters. From an 88-year-old Jewish grandmother, to Rashid a hip-hop loving teenager from the Bronx, the manipulation of her voice is what truly brings her characters to life. On the NPR broadcast of her TED Talk, the visuals are deemed inessential, 
furthering the power of the radio in conveying her message of stereotype and identity play through voice. Sarah Jones alerts us to the troubling social and cultural perpetuation of stereotypes decoded from the voice while simultaneously asking us to question our own understanding of the self-voice-body relationship. Like Sarah Jones, the Toronto soundwork women featured in this thesis work perceive the power of the amplified voice to extend the self through sound. The human voice -in particular here the publicly amplified voice - as an extension of the body binds it to our understanding of the self and our performative identities from a phenomenological and existential perspective.

Feminist philosophers, performance artists, historians and media scholars alike have analyzed the ways in which our cultural structures function to both confine women to their objectified passive bodies (visual media) and to estrange them from their bodies (radio). ${ }^{5}$ Through the lens of feminist phenomenology in conversation with the experiences of Toronto's women radio announcers and podcasters, we seek to reconcile the mediated body with the self - like Sarah Jones in her performance work - to understand how gender identity is experienced in our modern world. In contrast to early media theories of disembodiment and play-acted voice through electronic mediation, women in Toronto's modern soundwork industry experience their radio voice as an undeniably embodied extension of self. Whether inadvertently or directly influenced by second and third wave feminist ideals, these radio and podcasting women simultaneously experience their voice as an extension of self while in the same moment are aware of the performative aspect of their concept of self as an ongoing construction which fluctuates between levels of authenticity and dramaturgical production. Key themes are brought up 
by these women on the subjects of authenticity, relatability, and conversationality, which constitute both their understanding of the 'real' self extended through their soundwork while simultaneously harkening back to their conception of the 'ideal' radio voice. This complex relationship between gendered identity production and self-perception furthers desire in our media industry for a de-gendering of the 'ideal' radio voice toward a perception weighted on the authentic, relatable and conversational 'real' presentation of the self on the microphone.

\section{The Radio Presenter: Authentic Voice and the Intimate Self}

The desire for the authentic in communication and media is particularly sought after in the soundwork realm because of radio's intimate aesthetic as a voice-driven medium. This intimacy makes radio the ideal medium for the circulation of the emotional expression and personality conveyed through the human voice. This circulation of emotional expression and personality is required for authenticity to be perceived as such by the listener. When the electronics and digital distribution models are stripped away, the voices of radio and podcasting are embodied human beings/persons sharing their unique perspectives and personalities with the public. The English word 'person' comes

from the Greek 'per sona' meaning 'through sound ${ }^{6}$ expressing the human understanding of the sound of the voice as an indisputable part of the embodied self. Whether our voice is carried across a stage or through sound media, the sonorous amplified voice is a powerful and intimate extension of the self. 
When asked whether their voice was an extension of their 'true' or 'authentic' self, it quickly became clear - from the speedy responses of participants sounded with conviction - that soundwomen today perceive their radio voices as clear presentations of their authentic self. One participant responded, "it's $100 \%$ an extension of who I am, I know I sound the way that I sound"7 a self-assured response echoed by their fellow Toronto broadcasters. It is important to re-iterate here that the women participants in my study are neither news exclusive presenters nor primarily voice actors in fiction-based soundwork. All the radio and podcasting women here are first and foremost music announcers or show hosts in non-fiction or talk format soundwork. Though select participants do voice work across many media formats from commercials and fiction work to top 40 radio, the presentation format of music announcing and talk based soundwork are the foundation for the responses given. By responding to the question of radio voice as " $100 \%$ an extension of who I am" $"$ indicates not only a strong understanding of one's performative self-identity but an understanding of the mediated voice as an extension of the self through technological mediation.

Self-perception of voice referenced in studio headphones or played back on air is often experienced as alien to the speaker upon first listen. This discomfort with voice playback is a common reaction to hearing one's own voice without the internal resonance production of the physical body. Vocal self-perception is defined as "the physical and psychological experience of one's own voice". ${ }^{9}$ It is an experiential monitoring of the physical aspects of vocal production and a reflection of individual understanding of our vocal identity. Toronto soundwork women echo this experience of the voice as 'alien' or 
'other' as common in the initial stages of their career. "I had this reaction like 'that's cringy", one participant remarked, "I think that's the way a lot of people respond to their voice." ${ }^{\prime 10}$ While some participants indicate a continued 'alien' experience of their voice even after several years on the air, others expressed a shift toward a certain level of comfort and acceptance in hearing their own amplified voice, "[it's] a process of just hearing it more, you really do realize what you rely on, what your weaknesses are and you learn to adapt." ${ }^{11}$ Just as any sudden new discovery or revelation in self-identity, the extension of the voice through technology takes time to accept as part of our performative gender identity and material self. The presenter experiences their voice as both subjective and objective, as listener and as speaker. This comfortability with their radio voice in relation to their perception of self is not only an understanding or recognition that 'yes this is the sound of my voice' but an understanding of the radio voice as a part of their gender performativity and identity construction.

The experience of the radio voice as an extension of the self and thus the material body is certainly one of considerable debate. We must take the time to consider how the presenter perceives their voice in relation to the given tools of the microphone and various recording and broadcast technologies for the extension of their voice rather than a severing from the source. In Electrified Voices, sound scholar Barry Truax contemplates on the amplified voice, "is the source of the voice a real person or an inanimate loudspeaker or is it entirely synthetic; it can be all three at once," ${ }^{, 12}$ Sound artist Frances Dyson in "The Genealogy of The Radio Voice" argues further that much like the voice of the mind -our internal voice- the voice of authority on radio strives towards 
disembodiment. ${ }^{13}$ This triangulated experience of embodied, disembodied and reembodied voice cannot be left unobserved yet speakers themselves seem to experience their voice not simply as a sound emitted form a source but an extension of their material self. The voice as embedded in their physical form whether it be a 'live' broadcast or audio-on-demand, their voices are still their own. I don't wish to invalidate the ways in which technology can shape the voice through equalization, compression and effects processing to strengthen or enhance before it goes to broadcast but I want us to question if adding slight technological enhancements to ours voices is all that unlike receiving vocal coaching to enhance certain frequencies or address certain vocal crutches - ums, awes, likes, inflections, or ticks - within each of our unique vocalic frameworks. The voice as sound may be conceived as disembodied through media but the voice is always still embodied. Without the body there is no voice.

\section{"How Does One Sound Authentic?"}

Throughout my interviews with Toronto soundwork women, the theme of authenticity arose as a key term in the understanding of their voice and their self-identity. In discussing themselves and their voices as authentic, they are in turn discussing women-identified voices in soundwork as authentic. As Griffiths argues in Feminisms and The Self, "The construction and maintenance of 'I' or 'self is both with and through others (you, they, we)." ${ }^{\prime 14}$ That is to say, although a singular group of women cannot speak for the industry as a whole, repeated acuities brought to light by each of the women interviewees gives us a greater understanding of how these self-perceptions are shifting and coalescing within our current modern media realm. Furthermore, in proclaiming their 
voice is an extension of the self, they are proclaiming that radio voices are in turn an extension of gender identity. The reoccurring theme of 'authenticity' throughout the study brings us back to a particular question hinted toward in our earlier chapter on 'ideal' radio voice; the question being 'how does one sound authentic?'

Authenticity is a subject of considerable philosophical debate yet the term is frequently used in everyday social interaction. As Trilling notes in his 1972 book Sincerity and Authenticity, "The word authenticity comes so readily to the tongue these days." 15 As a term, which arose quite often in my interview series with Toronto's female radio and podcasting voice talents, 'authentic' in soundwork connects to conversationality, comfort on the mic, and a 'natural' presentation of their personality. If someone sounds 'unnatural' or 'forced' then they are less likely to be judged as 'real', or 'true to the self' ${ }^{16}$ Authentic emotion through speech and voice is proven to be aurally recognized as distinct from play-acted emotions. ${ }^{17}$ Researchers Drolet, Schubotz and Fisher in their 2011 study on the subject found that participants reacted more to authenticity than play-acted emotion. Interestingly enough, their study relied on voice recordings from a German radio archive. The use of radio archive material for their research furthers the connection between soundwork and authentic presentation. This greater response to authentic presentation is echoed by the desire for authentic voices in today's radio and podcasting industries.

Desire for authentic voice and what constitutes sonic authenticity in soundwork was brought up in conversation with Toronto podcaster and writer De Laurentiis Johnson 
(DLJ): "the personality in their voice insinuates a sense of authenticity that they haven't been told how to sound, its welcoming to my ears, it sounds like I'm in a conversation with them". ${ }^{18}$ DLJ discusses authenticity as contingent not only on the conveying of personality by the speaker but as dependent on the reception of the voice as such by the listener. Digital radio personality Eleanor Grace echoes DLJ in a desire for "people who are just natural conversationalists. You listen to them and it just sounds like their talking with you and you're comfortable." 19 The immediate connection of authentic radio voice with a natural conversational style provides some clues toward an understanding of authentic voice as one that is talking directly with the listener, drawing on the intimate power of sound based media to create a sense of sincere dialogue. The notion of dialogue in turn relies on signal and response rather than the traditional radio model of one-way signal for the reception and dissemination of information. Of course today in our social media driven digital age, the radio broadcaster is often interacting with listeners via social media during any given 'live' broadcast. In the audio-on-demand format, show hosts will also interact with listeners via social media (though not in the same 'live' setting), can choose to read listener responses in later episodes and have the flexibility to take into consideration listener reactions when producing their next work.

Our modern Western media industry thrives on this call-response model of social media networks, furthering the desire for and acceptance of 'authentic' conversational voices because listeners feel like they really get to 'know' these radio broadcasters. They follow them on Instagram, they chat with them on Facebook and they listen to them in their cars on their drive home from work. As a broadcaster in her early years, Woodrow 
sees the "rise of social media" as clearly having "something to do with it." ${ }^{20}$ The "it' being the desire for authenticity in the voices we hear extended through radio and podcasting media. Woodrow goes on to say:

I think because we're so curious about looking into people's lives - like Instagram stars, Youtube stars, people that are regular everyday folk but their kinda famous - I think you want all your media to reflect that to some degree. So even though I'm in a very old [media] profession it has to keep up with the times and what's going on right now. Which is really that anyone could be popular or anyone could be famous so if you have anyone talking to you wouldn't you want them to sound like your friend? ${ }^{21}$

Their words echo the shift we are experiencing in soundwork toward a desire for a multiplicity of voices. Booming masculine and overly scripted voices of authority are no longer desired for local radio or conversational formats. But as the 'authentic' voice becomes a desired sound in radio and podcasting talent, an awareness of the performative aspect of authenticity comes into the presentation of the voice on air. In relation to her own voice Woodrow revealed, "I think I sound relatable beyond anything else because that's what I like in a broadcaster so I generally try to reflect that."22 The awareness of trying to 'sound' relatable and trying to 'sound' authentic indicates relatability on air as a desired trait while addressing the conscious awareness of performativity in the presentation of self-identity within the public sphere. This desire for the authentic and simultaneously awareness of performativity beg the question; how we can have a sense of what is 'authentic' unless we have a sense of a snapshot of who were truly are? Our feminist understanding of the self re-emerges here - whether subconscious or active in our gendered experience - toward a duality in our understanding of identity as momentarily fixed yet perpetually under construction. 


\section{Is This Performativity or Are We Just 'Putting it On'?}

Our modern understanding of 'authenticity' and its dubious use as a term of endearment for ones preferred radio personality brings into question whether our gendered material voices hold the sonorous secret to how we present 'authentic' sound. While the paralanguage and acoustic differences of the voice in authentic versus playacted vocal presentation continue to elude linguists and philosophers alike, the performative aspects of radio talk as a source of both entertainment and companionship create static interference between these two vocal characterizations. Through Butler's notion of gender performativity and time ${ }^{23}$ we can posit there is indeed no fixed identity behind the performativity of gendered voice but that identity is constituted by the very expressions that are said to be its results. Yet Benhabib further argues if there is no subject, no self behind these performative repetitions, how can we expect to "pull the curtain down"? ${ }^{24}$ As radio announcers and hosts, are we performing our authentic selves on air, a true extension of our material body and self-identity or are we simply 'putting it on?' This question cannot be ignored as we have found through the responses of soundwork women here, the radio voice is perceived as an extension of the material self. This extension is also seen as one of authenticity toward the conception of the 'ideal' radio voice regardless of gender, race, sexuality, or background. 'Do you alter or 'put on' a voice?' This question was asked of Toronto soundwork women to delve into their unanimous response to the concept of their voice as an extension of their authentic self. 
Resulting responses to this question are mixed. Compared to the overwhelming experience of the voice as an extension of the self, this particular follow up question brought forward split reactions. While some respondents indicated an awareness of a certain performative aspects to their presentation on air, others stayed true to their notion of their voice as a 'real' extension of their self-identity. As a music station announcer Woodrow didn't waiver in her response, "This generation of broadcasters, I feel like we've just had it drilled into our heads like just sound like you." ${ }^{, 25}$ Here Woodrow again leans toward a desire for a multiplicity of voices and away from dramaturgical radio stereotypes. While Woodrow sees her voice as grounded in a true sense of self, in the same interview she also recalls her awareness of the performative aspects of her voice, "when you recognize that you are someone to do an impression of, you kind of it can be very tricky to not fall into a trap of doing an impression of yourself. ${ }^{, 26}$ Being able to perceive her voice as an extension of the self while also being able to hear the sonic characteristics of her voice is integral for the radio announcer. While authenticity is seen as 'ideal' for the radio voice, listening to your voice and developing a comfort with vocal self-perception furthers the speaker's ability to present as authentic on the microphone because they are no longer actively distracted by their own voice referenced back in their headphones.

Authenticity carries deep ties to notions of power and of self-knowledge within the unconscious. Greek ancestry of the word 'authentic' comes from 'authenteo', which in short means to have full power over. ${ }^{27}$ There is certainly power in the understanding of the amplified voice through technology. The mixed response from soundwork women on whether they are 'putting on' a voice can be traced back to their comfort with their own 
radio voice as an extension of the self along with their self-knowledge of identity as performative in the Butler-esque sense, particularly in public space. Swing host and radio personality Dani Stover explained it as such; "I don't really think of it as two separate voices although I'm very aware they are... because I work in an industry where it's encouraged to be yourself and share personal experience I don't see a big disconnect." ${ }^{28}$ As more and more radio announcers and voice talents are encouraged to 'be themselves' on air, the yesteryear play-acted presentations we used to hear in soundwork - the Ronny' radio voice or sultry radio vixen - are being erased from the airwaves. As we look to the future of soundwork and radio broadcast in the digital age, an understanding of radio voice as an extension of the self becomes integral to the speakers ability to be perceived as authentic. Listening to your voice - outside of the chambers of your own body - as it extends over the air - aids in further understanding the ways in which others may perceive your voice. This desire to understand the complex constructions of voice identity within our current digitally driven social and cultural context in turn reflects on the ways in which modern soundwork women are challenging the historically masculine heteronormative expectations of the North American radio soundscape.

\section{Electrified Voices and The Changing Digital Self}

When discussing the realm of soundwork - be it terrestrial or digital radio, podcasting or alternative audio-on-demand distribution- the effect that our digital media culture is having on our perceptions of voice and our connection or extensions of embodied self through technology is fundamental to the understanding of the electrified voice as an extension of the self-identity. Truax argues that "contemporary audio media rely on exact repetition, the position of ads within the highly structured programming 
flow, and instead of the redundant and copious forms of orality, the compact form of the contemporary sound byte. ${ }^{29}$ Media producers argue that audiences have shorter attention spans than ever before, we have more distractions around us than ever before, and are using media as an accompaniment to our multitasking world rather than "a singular primary focus. ${ }^{30}$ While there is certainly some truth in the increased immediate distractions in our day-to-day lives - be it our smartphone or the copious billboards that litter our city centre - soundwork professionals in what are considered millennial and post-millennial demographics are growing tired of this static radio model. Indeed our traditional radio soundscape continues to be dominated by these structured sound byte forms of commercially driven programming but new forms of talk-driven sound media are beginning to reemerge in the mainstream. The increasing visual distractions in our digital world are not simply creating distracted consumers but are in turn creating consumers looking for a deeper more in depth means of escape from the sometimes over stimulating visual world.

This desire for an escape from our visual-driven fast-paced commercial world has led to a call for slowed down connection, for human voices that not only sound authentic but sound like a friend. Someone you want to spend some time quality with. This desire for the authentic voice could potentially be connected to the rise in podcasting popularity over the last decade. ${ }^{31}$ In Understanding Media, Marshall McLuhan discusses a shift in radio as we entered our television-dominated era from "an entertainment medium into a kind of nervous information system." ${ }^{, 32}$ McLuhan positions radio as "an extension of the central nervous system that is matched only by human speech itself., ${ }^{33}$ McLuhan did not 
live to witness our intimate connection with technology that has grown through our contemporary digital culture. Radio is a medium and it is a message but in its modern soundwork form it extends human speech itself as an expansion of our embodied voice. The individualistic listening experience and ease of content distribution as a result of the digital shift, creates a new opportunity for radio to re-explore its identity outside of the sound byte model and toward a public amplification of our material selves.

On the topic of chat-genre/non-fiction podcasting and our shifting digital culture, the majority of participants indicated that podcasting is influencing the types of voices and volume of diversity of voice in the radio broadcast sector. As someone who has worked through this shift in her over ten years as a radio host, Dani Stover says it's time for a longer form of human connection on radio:

I do think that podcasting, chat genre is influencing broadcast media and radio. I think if radio stations were smart they'd jump all over that. 10 years ago (laughs). I think radio is going through a bit of an identity crisis and I think we've gone from a the audience just wants to listen to music all the time to maybe we should let people talk about more and get that human connection... ${ }^{34}$

While others noted the differentiation between the two media forms - "Podcasts are their own beasts and it always will be" ${ }^{, 35}$ - the influence that chat-genre podcasting is having on the radio industry is just beginning to take hold. "It democratizes what we do as broadcasters. ${ }^{36}$ As a radio announcer Siobhan Woodrow is excited by the potential for digital integration and new forms of radio programming sees a shift happening but is unsure when and if long form talk will return to commercial radio outside of the current politics and news heavy AM talk radio format. "I mean talk radio is exclusive to like politics there isn't a young hip chat show happening right now. Is there space for it? 
Yeah, but it will take a long time. ${ }^{37}$ This desire for human connection in our digital world further indicates a shift toward a de-gendering of 'ideal' radio voice, an opening up of the airwaves, of our headphones and stereos listening out for human connection rather than booming voices of authority.

Over two decades ago - almost as if it was prophecy - soundscape composer and philosopher R. Murray Schafer spoke of the untapped potential for radio, "If modern life is too fast paced, radio might find a new vocation if, instead of increasing its tempo as it appears to be doing, it assisted man in slowing down by reinforcing once again the natural rhythms of life." ${ }^{38}$ High fidelity recording, digital broadcast and multi-platform distribution means there is no longer a need for the sharp booming play-acted voices of yesteryear radio. The increased intimacy of clear digital and FM audio circulation gives the mediated voice back its direct correlation to the embodied self who emits it. Radio voice is not disembodied. Whether inadvertently or directly influenced by contemporary feminist theory, Toronto radio and podcasting women simultaneously experience their voice as an extension of self while in the same moment are aware of the performative aspect of their concept of self as an ongoing construction which fluctuates between levels of authenticity and dramaturgical production. A desire for authentic, conversational and relatable voices in our media opens up the airwaves toward a rethinking of what voices are deemed 'ideal' for broadcast outside of our current patriarchal soundscape. Through our growing familiarity with the ways in which embodied voice is electrified and extended through our digital networks, a reconciliation of the embodied voice with the material body is taking place. In a major city such as Toronto this means voices in 
soundwork must diversify toward a multiplicity of voices to reflect our embodied population across race, gender, sexuality and cultural background. By re-embodying our voices, we breathe new power into radio and sound based work. No longer disembodied, the identities behind the radio voices cannot be ignored. 


\title{
Conclusion
}

\section{TOWARD A MULTIPLICITY OF VOICES...}

\begin{abstract}
"I think years ago, women felt they had to have a certain whatever to their voice and they had to be a little extra girly and they had to say things a certain way. And now people are just a lot more honest and when you get that way, your real voice comes out." - Kat Callaghan, Radio Announcer
\end{abstract}

As more women find their voice on radio - be it traditional or digital - the act of listening and hearing other women's voices in the radio soundscape empowers and inspires others to engage. The gendered radio soundscape cannot anticipate change until we make that change audible. The amplified voice on air extends from the body as sound and carries with it our unique vocality from which gender, age, culture, emotion and experience are decoded and received by the human ear. ${ }^{1}$ The disembodied woman of media is fiction. Our voices may be experienced differently played back through sound technology, but that doesn't make it any less a part of our embodied experience and selfidentity. Every voice you hear is an opportunity for shared human connection, an opportunity for aural experience of the embodied self and the embodied other. As Western culture moves toward a changing digital broadcast soundscape, listeners and producers alike need to stop asking which radio voices are preferred and start asking why.

The physical characteristics, the way in which they are perceived, the function and meaning, and the affective qualities of the gendered mediated voice in soundwork are found within the lived experiences of the women who share their voices with Toronto each and every day. Perceptions of low/deep/masculine voice as preferred or 'ideal' for soundwork are beginning to become a standard of the past in Canada and the desire for a multiplicity of voices on air is clear. Yet there is still much work to be done before an 
audible shift can be heard within the Toronto soundscape and across the country. The continued study of gendered voice in broadcast media is an integral step toward the understanding of gender representation both on and off the microphone.

\section{Say Goodbye to 'Ronny' Radio}

Even as women situate themselves as key players within the radio soundscape, their voices sit further down on the pitch scale leaning toward a gender ambiguity and lower 'feminine' pitch range. The study of gendered voice as sound becomes undeniably valuable as it lays at the most basic level of our human experience. Hearing - or so it is claimed - is the last sense to pass on at the end of life. ${ }^{2}$ Every voice you hear is the voice of a mother, a daughter, a sister, a friend. Perceptions of lower or more 'masculine' coded voice as 'ideal' for soundwork are beginning to fade, but the answer to, "what is the 'ideal' radio voice?” continues to change with our political and cultural politics. Recontextualized in "Chapter Three", an exploration of the 'ideal' radio voice along with pitch and perception within the Toronto broadcast soundscape uncovered a lingering of bias toward higher pitch voices or 'feminine' voices on air while indicating a shift toward a more ungendered focus on the personality and 'authentic' presentation of the voice. The top four traits to trend among participants were 'authentic', 'conversational', 'relatable', and 'clear'. Not only are these top traits gender ambiguous, they are also subjective sonic traits that can be considered unrelated to pitch and any obvious or clear material analysis. These findings can be used to encourage professional radio management to listen to talent demo reels without focusing on gender and pitch but rather orienting their ears for personality and conversational presentation to appeal to our desire for human connection. 
This research could also be of use to post-secondary educational institutions when creating their media production course materials for broadcast presentation to promote a diversity of voices to pursue broadcast rather than perpetuating the lower radio voice 'ideal' of the past.

While half of participants indicated a perception of their voice as lower or deeper, half also indicated a sense of their voice as sonically 'average' in relation to other voices on air. Five participants from the interviews conducted were chosen for pitch analysis to further explore how our self-perception differs or compliments computer-assisted aural analysis. The unanimity of lower range pitch among all five woman-identified radio announcers shows that the radio industry, or at least the managers and programmers hiring on air talent, are still perpetuating the long standing inclination toward 'deeper' or 'masculine' voices as the radio 'ideal'. Although our perceptions of what is deemed to be an 'ideal' radio voice are changing along with increased access to a multiplicity of voices through podcasting, women's voices in public space continue to be subject to higher scrutiny and judgment than their male counterparts.

The experiences shared by Toronto radio women indicate a desire for different accents and personalities on air - at least here in Toronto - with the 'ideal' radio voice shifting toward a sphere of gender-ambiguity outside the realm of our traditional binary gender norms. We can see the influence of Judith Butler's theory of performativity in relation to the construct of gender through voice and the repetitions of 'feminine' and 'masculine' vocalities. The awareness of identity as "performatively constituted by the 
very expressions that are said to be its results" ${ }^{3}$ can be heard in the desire for a more 'authentic' style of performance over the overtly 'phony' over-performed masculine Ronny radio voice. The historical biases experienced by women toward the 'feminine' or higher pitched voice on air continues today yet in a much different form than experienced by women in the early years of radio. ${ }^{4}$ Future research is required to monitor whether this shift toward a consistently lower female or midrange male voice will expand to include a wider range of sonorous voices or continue toward a uniformity in sound across gender, race, culture and other sounding factors of our constructed identity.

\section{Gendered Voice and The Politics of Power}

The gendered experiences of women in soundwork highlight the continued rule and restriction when it comes to women's voices amplified. With a focus on the Toronto soundscape, experiences of discrimination and negative comment towards their own voices indicate continued patterns of the patriarchal ear similar to those experienced by women over twenty years ago as outlined by Rosalind Gill. ${ }^{5}$ It is through the repetition of language, gendered stereotypes, radio tropes and other performative acts that gender inequity persists within a masculine/feminine power dynamic of our own construction. ${ }^{6}$ Thrusting us back into the sounding body, the contemporary feminist phenomenological approach compels us to note that speech is not voice for the voice is sound and "speech is its essential destination." ${ }^{, 7}$ It is the personal lived experiences of the women and voice talents targeted by these biases and perceptions that situate our understanding of how amplified voices are shaped through historically gendered and performative work. 
Power plays an important role in the experiences of women in soundwork, as power is tied directly to the amplified voice. Who is deemed to have the authority or 'ideal' voice for amplification through soundwork is still an ongoing struggle for the professional broadcaster. 'Uptalk', 'vocal fry', and higher pitched voices received particular note as traits discriminated against related to the female or 'feminine' voice on air. It is no coincidence that these vocal traits share a common thread of historical association toward the younger less educated 'feminine' voice typically linked to the misogynist 'valley girl' or 'blonde bimbo' stereotype. Hyper feminized stereotypes - used to take away any authority from the girl, the woman, and the feminine - are voices perceived as outside of or uncharacteristic of the heteromasculine sounds that dominate our media soundscape. Drawing on the experiences of women in the Toronto soundwork industry, the value of their lived experiences reminds the listener of the human intersubjectivity and empathy involved in listening to the amplified voice. In our Trumpian global political landscape, the post-feminist dream of gender equality is far from reality. The results of these inequalities and power dynamics mean women's voices are listened to in a vastly different way than one listens to the male or masculine voice. Until we begin to hear a multiplicity of voices on in our broadcast landscape, we can only expect history to repeat itself.

\section{The Self through Sound, Embodiment in Radio}

Feminist philosophers, performance artists, historians and media scholars alike have analyzed the ways in which our cultural structures function to both confine women to their objectified passive bodies through visual media and to estrange them from their 
bodies through sonification on radio. ${ }^{8}$ The Toronto soundwork women featured in this thesis work reconcile the power of the amplified voice as an extension of the self through sound. When asked whether their voice was an extension of their 'true' or 'authentic' self, it quickly became clear that radio women today perceive their voices as clear presentations of their authentic self. The human voice -in particular here the publicly amplified voice - as an extension of the body binds it to our understanding of the self and our performative identities from a phenomenological and existential perspective. Our sense of self is intimately tied to our voices and the heard voices of others. Consideration of the vocal body allows us to question the relation of the "biological and the cultural, the somatic and the symbolic" in the assembly of bodies and subjectivities. ${ }^{9}$ A greater understanding and appreciation for the uniqueness of the material and speech-language voice as an intimate extension of the gendered self can open the airwaves towards a multiplicity of voices across gender, race, ability and sexuality.

Key themes were brought forward by Toronto soundwork women on the subjects of authenticity, relatability, and conversationality, which constitute both their understanding of the 'real' self extended through their soundwork while simultaneously harkening back to their conception of the 'ideal' radio voice. The reoccurring theme of 'authenticity' throughout the study brings us back to a particular question hinted toward in our earlier chapter on 'ideal' radio voice; the question being 'how does one sound authentic?' The immediate connection of authentic radio voice with a natural conversational style provides some clues toward an understanding of authentic voice as one that is talking directly with the listener, drawing on the intimate power of sound 
based media to create a sense of sincere dialogue. A desire for authentic, conversational and relatable voices in our media opens up the airwaves toward a rethinking of what voices are deemed 'ideal' for broadcast outside of our current patriarchal soundscape. We must take the time to consider how the presenter perceives their voice in relation to the given tools of the microphone and various recording and broadcast technologies for the extension of their voice rather than a severing from the source. This triangulated experience of embodied, disembodied and re-embodied voice cannot be left unobserved yet speakers themselves seem to experience their voice not simply as a sound emitted form a source but an extension of their material self. The voice as embedded in their physical form whether it be a 'live' broadcast or audio-on-demand, their voices are still their own. Without the body there is no voice. No longer disembodied, the identities behind the radio voices cannot be ignored. How might the conversation of embodiment in the digital space change the ways in which we engage with one another online? The reembodiment of the voice in media opens up the possibilities for a new approach to the ways in which we engage with our media across multiple platforms from radio to television and online social media engagement from an ear-oriented perspective.

\section{Gender Equity in Canadian Media}

"When I started working at my radio station I was the first female radio host to ever work there and this was only in '92! Things are changing with more women getting into the business. And I think that's fantastic." - Stacey Englehart, Radio Announcer

The experiences of women in Toronto's soundwork industry are required to develop a greater understanding of the socio-cultural frameworks that shape our 
perceptions of 'masculine' and 'feminine' voice influenced in turn by their media representation. How we can move toward an equitable broadcast soundscape and what that future may sound like is still unclear but there are certainly clues hidden within the sonic materials and perceptions of gendered radio voice. This research contributes to the broader feminist media discourse on radio and voice by reconciling the gendered body with the mediated material voice. Discussion of gendered voice in radio - through the experiences of Toronto radio and podcast talent - opens up further conversation surrounding diversity and gender equality in the Canadian media landscape. While select experiences of women on the mic were collected and shared through this work, future research into women in radio programming and management could provide additional experiences and perspectives on the inner workings of the radio industry when it comes to gender representation in Canadian media.

Although no definitive data for the Canadian radio industry has been published at this time, we can look to our neighbors in the south to give us an idea of the disparity in gender representation within the radio programming and management sector. According to the 2017 MIW Gender Analysis Study released by the Mentoring and Inspiring Women in Radio Group, women currently program only $10.7 \%$ of all stations in the United States, which compares to the same number ten years ago. ${ }^{10}$ Would we hear more feminine or female voices on air if more women were in program management positions? Would we hear a greater sonic variety of women's voices on air if more women were in these positions of power? It is certainly plausible and as we see 
more women take on leadership and programming roles in radio, further research into these questions could dramatically change our Canadian media soundscape.

\section{Toward a Multiplicity of Voices}

"I don't think you're going to go on without a diversity of voices for much longer and were speaking from quite a diverse city (Toronto)... I think we have to be careful not just with racial diversity visually but we have to make sure immigrant voices are a lot more heard here." - Susan McReynolds, Radio Host and Producer

Once you start to listen, it is not difficult to locate the voices excluded from the radio soundscape. In a city as diverse and multicultural as Toronto, once you open your ears critically to the media soundscape, you have to wonder, where are the immigrant voices, the voices with accents, the voices of disability, the voices of our city? It is crucial to note that America stands out for its steady and significant climb from $29 \%$ female presenters in 2000 to their 2015 Global Media Monitoring Report of 44\%, a 15 percentage point reduction of the gender gap in 15 years. According to the same report, women were over-represented as television presenters in Canada at $78 \%$ to $22 \%$ for men respectively. ${ }^{11}$ This is not particularly surprising, as the medium of television has traditionally valued the physical appearance of women as TV news anchors and reporters. Overwhelmingly, female Canadian television announcers were young with $51 \%$ between the ages of 19-34 and 29\% between 35-49, showing indications of age bias in the industry. While gender representation in media has seen increased numbers up until 2010, "visible minorities" still make up less than $10 \%$ of the American radio news workforce. ${ }^{12}$ These numbers have sparked media discussion around diversity in our Canadian media landscape, creating a call to commercial media in particular to address racial inequities 
among their employees on and off screen. ${ }^{13}$ Managers may sum it up to "minorities just don't apply here," ${ }^{14}$ but as ear-oriented scholars we must question how sonically diversifying the voices we hear on air could change the culture around who applies and who pursues a career in Canadian broadcast. Up until recent works by sound scholars such as Art M. Blake, Gustavus Stadler and Jennifer Lynn Stoever the relationship between race and sound has been quiet. ${ }^{15}$ As Stoever notes, "sounds never really lose their referent to different types of bodies despite being able to operate independently of them." ${ }^{, 16}$ Further work must be done to address the intersections of race and gender in relation to modern-day radio and the amplified voice to address the experiences of racialized women within Toronto's white-dominated patriarchal soundscape.

A final question during my interview series with Toronto's women-identified radio and soundwork talent brought forward a call to the Canadian radio industry to take on a sound that reflects the culturally diverse global network in which we live our embodied digitally engaged Canadian reality. "Are the voices we hear in radio and soundwork changing?" This is a simple question but one that sparked a desire for change in what is perceived as an outdated medium with bursting potential for a new generation. One participant's answer stood out in saying, "I really want to say it's changing so, so badly. And I don't know that I would be wrong but I think it's going to be a slow burn." 17 As one of the youngest participants, Woodrow remains hopeful that the commercial radio industry she is so passionate about will embrace the multiplatform digital culture of her generation to take new risks on programming and branding strategy. "I think people don't like to take risks because the industry doesn't have a lot of money to play around with. So 
when something is tried tested and true you go with what's been done over and over again." ${ }^{, 18}$ But as the broader political soundscape around us begins to change - from the Black Lives Matter Movement to the Women's March on Washington- the voices we hear in our sound media need to reflect the Canadians waiting to hear their own voices echoed back. The move toward a multiplicity of voices is a slow uphill battle within our patriarchal past and capitalist present. Through our growing familiarity with the ways in which embodied voice is electrified and extended through our digital networks, a reconciliation of the embodied voice with the material body is taking place. In a major city such as Toronto this means voices in soundwork must diversify toward a multiplicity of voices to reflect our embodied population across race, gender, sexuality and cultural background. Powerful and rising, these collective amplified voices of lived experience in Toronto's gendered radio soundscape reverberate outward as a call for change. Whether this call will be answered, only time will tell. 


\section{ENDNOTES BY CHAPTER}

\section{Endnotes for Introduction}

${ }^{1}$ Donna Halper, "Speaking for Themselves: How Radio Brought Women into The Public Sphere" in Radio Cultures: The Sound Medium in American Life, edited by Michael C. Keith, 7793, (New York: Peter Lang, 2008).

${ }^{2}$ Ibid, 80 .

${ }^{3}$ Kathy Walsh, Jane Suiter and Orla O'Connor, Hearing Women's Voices? (Dublin, Ireland: National Women's Council of Ireland and Dublin City University, 2015), 35.

${ }^{4}$ The Global Media Monitoring Project 2015. http://cdn.agilitycms.com/who-makes-thenews/Imported/reports_2015/highlights/highlights_en.pdf. 4.

${ }^{5}$ Val Maloney, "What's Next for 2017: Radio" in Media in Canada. (Published January 6, 2017) http://mediaincanada.com/2017/01/06/whats-next-for-2017-radio/.

${ }^{6}$ Don Ihde, "Introduction to the Original Edition" in Listening and Voice: Phenomenologies of Sound, $2^{\text {nd }}$ Ed. (New York: SUNY Press, 2007), xx.

${ }^{7}$ Adriana Cavarero, For More Than One Voice: Toward a Philosophy of Vocal Expression, Trans. by Paul A. Kottman, (Redwood City, CA: Stanford University Press, 2005), 12.

${ }^{8}$ Christine Ehrick, Radio and The Gendered Soundscape: Women and Broadcasting in Argentina and Uruguay, 1930-1950, (New York, NY: Cambridge University Press, 2015), 7.

${ }^{9}$ Michele Hilmes, "The New Materiality of Radio: Sound on Screens" in Radio's New Wave: Global Sound in a Digital Era, Edited by Jason Loviglio and Michele Hilmes, (New York, NY: Routledge, Taylor \& Francis Group, 2013), 60.

${ }^{10}$ Jason Loviglio, "Sound Effects: Gender, Voice and the Cultural Work of NPR" in The Radio Journal International Studies in Broadcast and Audio Media, no. 5 (July 2008), 67-81, https://doi.org/10.1386/rajo.5.2-3.67_1.

${ }^{11}$ Ibid, 67.

${ }^{12}$ Ehrick, "Introduction" in Radio and The Gendered Soundscape. (2015), 5.

${ }^{13}$ Ihde, Listening and Voice: Phenomenologies of Sound, $2^{\text {nd }}$ Ed. (2007).

${ }^{14}$ Linda Fisher, "Feminist Phenomenological Voices" in Continental Philosophy Review, no. 43, 83-95, DOI 10.1007/s11007-010-9132-y, (April 2010), 83.

${ }^{15}$ R. Murray Schafer, The Soundscape: Our Sonic Environment and the Tuning of the World. (Rochester, VT: Destiny Books, 1993), 275.

${ }^{16}$ Ihde, Listening and Voice (2007), 20. 
${ }^{17}$ Rosalind Gill, “Justifying Injustice: Broadcasters' Accounts of Inequality in Radio" in Women and Radio, Edited by Caroline Mitchell (2000), 137-151. Gill uses a discourse analytic approach to examine gender inequality in the UK radio industry through interviews with five male DJs. The verbatim interview quotations and findings play a central role throughout the article.

${ }^{18}$ Anne Karpf, "How Men and Women's Voices Are Changing (and Why)" in The Human Voice: The Story of a Remarkable Talent, (London: Bloomsbury, 2007), 172-181. Karpf and Loviglio (2008) both indicate a sonic shift in women's voices over the past fifty years in the Western world. With a focus on broadcast voices in particular, they claim that women's voices are getting deeper.

${ }^{19}$ Annette Schlichter, "Do Voices Matter? Vocality, Materiality, Gender Performativity" in Body \& Society, vol. 17, no. 1, 31-52, (2011). See also Linda Fisher, "Feminist Phenomenological Voices" in Continental Philosophy Review, no. 43, (April 2010), 83-95.

${ }^{20}$ Ehrick, Radio and The Gendered Soundscape, (2015), 7.

${ }^{21}$ Philip S.J. Weston, Michael D. Hunter, Dilraj S. Sokhi, Iain D. Wilkinson, and Peter W.R. Woodruff, "Discrimination of Voice Gender in The Human Auditory Cortex" in NeuroImage, no. 105, (October 2014), 208-14.

${ }^{22}$ Michele Hilmes, "Disembodied Woman" in Radio Voices: American Broadcasting, 1922-1952, (Minneapolis, MN: University of Minnesota Press, 1997), 130-50.

${ }^{23}$ Gill, "Justifying Injustice" in Women and Radio, (2000).

${ }^{24}$ Paul Warren, Uptalk: The Phenomenon of Rising Intonation, (Cambridge: Cambridge University Press, 2016).

The social and cultural phenomena surrounding uptalk and other 'feminine' coded speech sets has led to notable recent publications such as Warren's comprehensive writings on uptalk published in 2016.

${ }^{25}$ Annette Schlichter "Do Voices Matter? Vocality, Materiality, Gender Performativity" in Body \& Society, no.17: 31-51, (March 2011), 45. For the influence of sound technology and the history of sound, see also Jonathan Sterne, The Audible Past: Cultural Origins of Sound Reproduction, (Durham: Duke University Press, 2003).

${ }^{26}$ Sue Thornham, Women, Feminism and Media, (Edinburgh: Edinburgh Univ. Press, 2010), 107.

${ }^{27}$ The historical entanglement between white supremacy and listening in the United States with attention to the intersectional relationships between race, gender, sexuality and class is brought forward in Jennifer Lynn Stoever, The Sonic Color Line: Race, and the cultural politics of listening, (New York: NYU Press, 2016).

${ }^{28}$ Here I use the term "whiteness" in relation to sound and radio as discussed by Derek W. Vaillant, in "Sounds of Whiteness: Local radio, racial formation, and public culture in Chicago, 1921-1935", in American Quarterly 54.1, 25-66 (March 2002). The term "color blindness" in relation to white sonic norms in post-1945 radio is further discussed by Stoever in "Chapter 5: Broadcasting Race" of The Sonic Color Line, (2016), 176-209. 
${ }^{29}$ Hilmes, "Disembodied Woman" in Radio Voices (1997), 130-50.

\section{Endnotes for Chapter One: Theoretical Frameworks}

${ }^{1}$ Don Ihde, "Introduction to the Original Edition" in Listening and Voice: Phenomenologies of Sound, $2^{\text {nd }} E d$. (New York: SUNY Press, 2007), xx.

${ }^{2}$ Ihde, Listening and Voice, (2007) and Judith Butler, Gender Trouble: Feminism and the Subversion of Identity (Taylor \& Francis, 1999).

${ }^{3}$ Caroline Mitchell, Women \& Radio (2000), Hilmes, Radio Voices (1997), and Ehrick, Radio and The Gendered Soundscape (2015).

${ }^{4}$ Cavarero, For More Than One Voice (2005), Andrew Kimbrough, Dramatic theories of voice in the twentieth century, (Amherst, NY: Cambria Press, 2011), and Karpf, The Human Voice, (2007).

${ }^{5}$ Virginia Madsen and John Potts, "Voice-Cast: The Distribution of the Voice via Podcasting" in Voice: Vocal aesthetics in digital arts and media, Edited by Neumark, Gibson and van Leeuwen, (Cambridge, Mass: MIT Press, 2010), 33-59.

${ }^{6}$ Jason Loviglio, "Sound effects: Gender, voice and the cultural work of NPR" in Radio Journal: International Studies in Broadcast \& Audio Media vol. 5, (2007), 67-81.

${ }^{7}$ Martin Heidegger, Being and Time, trans. by Joan Stambaugh, (Albany, NY: State University of New York Press, 1996). Edmund Husserl, The Crisis of European Sciences and Transcendental Phenomenology, (Evanston, IL: Northwestern University Press, 1970). and Merleau-Ponty, Phenomenology of Perception, trans. by Donald A. Landes, (London: Routledge, 2012).

${ }^{8}$ Ihde, Listening and Voice, (2007), 20.

${ }^{9}$ Ibid.

${ }^{10}$ Ibid, 44. In relation to the existential self, Ihde notes the phenomenon of listening as a fully embodied one. "I not merely hear with my ears, I hear with my whole body."

${ }^{11}$ Ibid, 185 . Ihde opens chapter 17 with this consideration; "in the beginning was voice and the voice was speech and the speech was Language."

${ }^{12}$ Linda Fisher, "Feminist phenomenological voices" in Cont Philos Rev, vol 42, 83-95, (Springer Science+Business Media B.V. 2010), 83.

${ }^{13}$ Judith Butler, Gender Trouble: Feminism and the subversion of identity, $2^{\text {nd }}$ ed., (New York: Routledge, 1999). On the theory of 'performativity' in relation to gender, 171-90.

${ }^{14}$ Simone de Beauvoir, The Second Sex, trans. by H.M Parshley, (New York: Alfred A. Knopf, 1975), 301. 
15 Judith Butler, Undoing Gender, (New York: Routledge, 2004), 177. This later work by Butler furthers their discussion of performativity in relation to normative gender roles and the resulting exclusions and othering that occurs when someone cannot emulate these norms.

${ }^{16}$ Annette Schlichter, "Do Voices Matter? Vocality, Materiality, Gender Performativity" in Body \& Society, vol. 17, no. 1, 31-52, (2011), 33.

${ }^{17}$ Ibid, 36.

${ }^{18}$ Derrida (1998: 20) (*get proper citation from Voice collection*). Auto affection: This experience of the effacement of the signifier in the voice is not merely one illusion among many since it is the condition of the very idea of truth ... This illusion is the history of truth.'

37.

${ }^{19}$ Schlichter, "Do Voices Matter? Vocality, Materiality, Gender Performativity", (2011),

${ }^{20}$ Ibid, 38.

${ }^{21}$ Fisher, "Feminist phenomenological voices", (2010), 87.

${ }^{22}$ Ibid.

${ }^{23}$ Michele Hilmes, "The New Materiality of Radio" in Radio's new wave: global sound in the digital era, by Jason Loviglio and Michele Hilmes, (New York: Routledge, Taylor \& Francis Group, 2013).

${ }^{24}$ Amy Allen, The Politics of Our Selves: power, autonomy, and gender in contemporary critical theory, (New York: Columbia University Press, 2008), 22.

${ }^{25}$ Michele Foucault, "Volume III: The care of the self”, in The History of Sexuality, (New York: Vintage Books, 1988-1990).

${ }^{26}$ Foucault, Madness and Civilization: a history of insanity in the Age of Reason, (New York: Vintage Books, 1988). Madness and Civilization brought forward the three axes which Foucaults work in The History of Sexuality hinges on. The domain of knowledge, a system of rules, and a model for relations to the self.

${ }^{27}$ Foucault, "Preface to The History of Sexuality, Volume II" in The Foucault Reader, edited by Paul Rabinow, (New York: Vintage Books, 2010), 338.

${ }^{28}$ Seyla Benhabib, Situating The Self: gender, community and postmodernism in contemporary ethics, (New York: Routledge, 1992), 1.

${ }^{29}$ Ibid, 4.

${ }^{30}$ Ibid, 215. 
${ }^{31}$ Benhabib, "Sexual Difference and Collective Identities: The New Global Constellation”, In The University of Chicago Press Journal, Vol 24: 2, (Winter 1999), 335-61.

${ }^{32}$ Morwenna Griffiths, Feminism and the Self: The Web of Identity. (London, US: Routledge, 2003).

${ }^{33}$ Joan W. Scott, "The Evidence of Experience" in Critical Inquiry 17, no. 4, 773-97 (1991), 773.

${ }^{34}$ Maurice Merleau-Ponty, The Primacy of Perception, and other essays on phenomenology of psychology, the philosophy of art, history and politics, edited by James M. Edie, (Evanston, Il: Northwestern University Press, 1964), 25.

${ }^{35}$ Ibid.

${ }^{36}$ Benhabib, Situating The Self, (1992).

${ }^{37}$ Griffiths, Feminism and the Self, (2003).

${ }^{38}$ Heidegger, Being and Time, (1996), 174.

${ }^{39}$ Hilmes, "The New Materiality of Radio" in Radio's new wave: global sound in the digital era, (2013), 60.

${ }^{40} \mathrm{R}$. Murray Schafer, The Soundscape: our sonic environment and the tuning of the world, (Rochester, VT: Destiny Books, 1993).

${ }^{41}$ Ibid, 275.

${ }^{42}$ Ibid, 272-275. The 'Keynote' is defined as "a musical term; it is the note that identifies the key or tonality of a particular composition. It is the anchor or fundamental tone and although the material may modulate around it. Examples might be the sound of the sea for a maritime community" (272). The 'Soundmark' is derived from the word "landmark to refer to a community sound which is unique or possess qualities which make it specially regarded or noticed by the people in that community" (274). And 'Sound Signal': is "any sound to which the attention is particularly directed. Contrasted by keynote sounds, in much the same way as figure and ground in visual perception" (275).

${ }^{43}$ Ibid, 123.

${ }^{44}$ Ibid, 145.

${ }^{45}$ Ibid, 4.

${ }^{46}$ Paddy Scannell, 'Review of books by le Roy Bannerman and A. Crisell', in Media, Culture and Society, vol. 10, no. 3, 385-88, (London: Sage, 1988). See also Scannell, Radio, Television and Modern Life, (Oxford: Blackwell, 1996). 
${ }^{47}$ Caroline Mitchell, Women and Radio: Airing Differences, (New York NY: Routledge, 2000), 4.

${ }^{48}$ Frances Dyson, "The Geneology of the Radio Voice" in Radio Rethink: art, sound and transmission, (Banff, Alta: Walter Phillips Gallery, 1994), 181.

${ }^{49}$ Hilmes, "Disembodied Woman" in Radio Voices, (1997), 130-50.

${ }^{50}$ Ibid, 133.

${ }^{51}$ Ibid, 141.

${ }^{52}$ Ibid, 142.

${ }^{53}$ Rosalind Gill, "Justifying Injustice: broadcasters' accounts of inequality in radio", in Women and Radio, edited by Caroline Mitchell, (2000), 137.

${ }^{54}$ Christine Ehrick, Radio and The Gendered Soundscape: Women and Broadcasting in Argentina and Uruguay, 1930-1950, (New York, NY: Cambridge University Press, 2015), 3.

${ }^{55}$ Ibid, 7.

${ }^{56}$ The disembodied voice in relation to women on air is discussed by Dyson in Radio Rethink and Hilmes in Radio Voices. See Hilmes "Disembodied Woman" in Radio Voices, (1997), 130-50. See also Frances Dyson, “The Geneology of the Radio Voice” in Radio Rethink, (1994).

${ }^{57}$ Adriana Cavarero, For More Than One Voice: Toward a Philosophy of Vocal Expression, Trans. by Paul A. Kottman, (Redwood City, CA: Stanford University Press, 2005), 522.

${ }^{58}$ The original title of Cavarero's work is the italian, A piu voci: per una filosofia dell'espressione vocale (Italy: Feltrinelli, 2003).

${ }^{59}$ Cavarero, For More Than One Voice, (2005), 176.

${ }^{60}$ Ibid, 208. Caverero states that, "the political essence of speech is rooted in the corporeal uniqueness of the speakers and in their reciprocal invocation."

${ }^{61}$ Ibid, 13.

${ }^{62}$ Andrew Kimbrough, Dramatic theories of voice in the twentieth century, (Amherst, NY: Cambria Press, 2011), 9.

${ }^{63}$ Ibid, 62 .

${ }^{64}$ Kimbrough, "The Voice in Phenomenology and Existentialism" in Dramatic Theories of Voice in the Twentieth Century, (2011), 67-106.

${ }^{65}$ Ibid, 21. 
${ }^{66}$ Walter Benjamin, The Work of Art in the Age of its Technological Reproducability, and other writings on media, edited by Michael W. Jennings, Brigid Doherty, and Thomas Y. Levin; trans. by Edmund Jephcott, (Cambride Mass: Belknap Press of Harvard University, 2008).

${ }^{67}$ Ibid, 264.

${ }^{68}$ Anne Karpf, The human voice: the story of a remarkable talent. (London: Bloomsbury, 2006), 10.

${ }^{69}$ Ibid, 24.

${ }^{70}$ Ibid, 25.

${ }^{71}$ Ibid, 175.

${ }^{72}$ This subject in relation to radio is also contextualized briefly by radio scholar Jason Loviglio in his article "Sound Effects: Gender, Voice and the Cultural Work of NPR" (2008).

${ }^{73}$ Roland Barthes, Image, music, text, trans. by Stephen Heath. New York: Hill and Wang, $1977.185 \& 188$.

${ }^{74}$ Norie Neumark, "Introduction: The Paradox of Voice" in Voice: Vocal aesthetics in digital arts and media, edited by Neumark, Gibson and van Leeuwen, (Cambridge, Mass: MIT Press, 2010), xvii.

${ }^{75}$ In reference to Pierre Schaeffer, Traité des objects musicaux. Paris: Editions du Seuil, 1966.

${ }^{76}$ Madsen and Potts, "Voice-Cast: The Distribution of the Voice via Podcasting" in Voice: Vocal aesthetics in digital arts and media, edited by Neumark, Gibson and van Leeuwen, 33-59, (Cambridge, Mass: MIT Press, 2010), 33.

${ }^{77}$ As conceptualized by R. Murray Schafer in The Tuning of the World: Toward a theory for soundscape design, (University of Pennsylvania Press, 1980).

${ }^{78}$ Madsen and Potts, "Voice-Cast" in Voice, (2010), 45. In the example of Rick Jarow's "Honoring Your Calling to Find Your Life's Work" podcast which features a female voice in the introduction which Madsen and Potts describe as "calm, midrange, and pleasant in a reserved manner."

${ }^{79}$ In Canada, many radio shows produced by our public broadcaster (CBC) are aired on CBC Radio before being packaged and distributed as a podcast online through their website cbc.ca and via podcasting applications, eg. iTunes, Google Play. See "CBC Radio Podcasts" on CBC/Radio Canada, Online. http://www.cbc.ca/radio/podcasts/.

${ }^{80}$ Jeffery Pittam, and Cynthia Gallois. "Predicting Impressions of Speakers from Voice Quality: Acoustic and Perceptual Measures" in Journal of Language and Social Psychology 5, no. 4, 233-47, (2016).

${ }^{81}$ Ibid, 244. 
${ }^{82}$ Karpf discusses gendered and cultural voice differences across the globe in Karpf, "How Men and Women's Voices Are Changing and Why" \& "Cultural Differences in the Voice" in The Human Voice: The Story of a Remarkable Talent, (London: Bloomsbury, 2007).

${ }^{83}$ Carol Ann Valentine and Banisa Saint Damian "Gender and culture as determinants of the 'ideal voice'" in Semiotica, vol. 71, no. 3-4, 285-303, (De Gruyter, 1988).

${ }^{84}$ Valentine and Saint Damain, "Gender and Culture as determinants of the 'ideal voice"”, (1988), 294.

${ }^{85}$ Ibid (295).

${ }^{86}$ Shelagh Davies and Joshua M. Goldberg, "Clinical Aspects of Transgender Speech Feminization and Masculinization" in International Journal of Transgenderism, vol. 9, no. 3-4, 167-96, (2006), doi:10.1300/j485v09n03_08.

${ }^{87}$ Ibid, 167.

${ }^{88}$ Ibid, 178.

${ }^{89}$ The $145-165 \mathrm{~Hz}$ range could be considered gender ambiguous as it shares crossover with the range 155-185 Hz (D\#3-G3) considered gender ambiguous by speech-language pathologist Christie Block at New York Speech \&Voice Lab. 2014. Accessed January 28, 2017. http://www.nyspeechandvoicelab.net/transgender/voice-feminization/. See table 3.4 for reference.

${ }^{90}$ See Pittam and Gallois (1986), Valentine and Saint Damain (1988), Davies and Goldberg (2006). See also The Journal of Voice (Elsevier, 1987-), a premiere journal dedicated to voice medicine and research.

${ }^{91}$ Samantha Warhurst, Patricia Mccabe, and Catherine Madill, "What Makes a Good Voice for Radio: Perceptions of Radio Employers and Educators" in Journal of Voice, vol. 27, no. 2, (2013), 217-24, doi:10.1016/j.jvoice.2012.08.010.

92 Gill, "Justifying Injustice" in Women and Radio, 137-151, (2000).

${ }^{93}$ Loviglio "Sound effects: Gender, voice and the cultural work of NPR" in Radio Journal: International Studies in Broadcast \& Audio Media vol. 5, no. 2, 67-81, (2007), 67.

${ }^{94}$ Christine Ehrick uses sound studies in her radio history work in Ehrick, Radio and the Gendered Soundscape, (2015). See also Kate Lacey, Feminine Frequencies: Gender, German Radio, and the Public Sphere, 1923-1945 (Michigan: University of Michigan Press, 1997), for a feminist perspective on the early years of the German Radio industry.

${ }^{95}$ Jason Loviglio. "Threatening and threatened, voices of women have defined public radio." Current. March 16, 2017. Accessed March 29, 2017.

http://current.org/2017/03/threatening-and-threatened-voices-of-women-have-defined-publicradio/. New research on NPR radio voice by Loviglio is ongoing.

${ }^{96}$ As Loviglio reiterates in his article "Sound effects: Gender, voice and the cultural work of NPR" (2007) and Anne Karpf argues in The Human Voice (2007). 
${ }^{97}$ Karpf, The Human Voice, (2007), 175-177.

${ }^{98}$ See Ihde, Listening and Voice, (2007) and Adriana Cavarero, For More Than One Voice, (2005).

${ }^{99}$ See Butler (1999) Schlichter (2011) and Fisher (2010).

${ }^{100}$ See sociolinguistic and voice studies; Valentine and Saint Damain (1988), Davies and Goldberg (2006), Pittam and Gallois (2016), Warhurst, Mccabe, and Madill (2013).

${ }^{101}$ See Neumark, Gibson, and Van Leeuwen (2010). See also Dmitri Zakharine and Nils Meise, Electrified Voices: Medial, Socio-historical and Cultural Aspects of Voice Transfer, (V\&R Unipress, 2013). Electrified Voices is a collection of articles that explore the subject of evoices aka "electrified voices" through an interdisciplinary approach.

\section{Endnotes for Chapter Two: Methods}

${ }^{1}$ GMMP, The Global Media Monitoring Project 2015. http://www.iawrt.org/sites/default/files/field/pdf/2015/11/GMMPsummary2015.pdf, (2016). 1.

2 Jonathan Sterne, "Being 'In the True' of Sound Studies" in Music, Sound and the Moving Image, no. 2., (Liverpool, UK: Liverpool University Press, 2008), 163.

${ }^{3}$ Government of Canada, Canadian Radio - Television and Telecommunications Commission (CRTC), "Radio AM/FM - Canada - All Languages", (Apr 2016), http://www.crtc.gc.ca/eng/publications/reports/BRAnalysis/radio2015/radio1.htm.

${ }^{4}$ CRTC, "Broadcasting sector overview" in Communications Monitoring Report 2016. http://www.crtc.gc.ca/eng/publications/reports/policymonitoring/2016/cmr4.htm\#a4.

${ }^{5}$ See Lori Beckstead, "Diversity on Air" Radio Installation Art (2016). The Government of Canada and the CRTC in the 2017-2018 year, are also set to publish the results of a study on how diversity in Canada is reflected in a multi-platform broadcasting environment.

${ }^{6}$ Toronto's soundscape continues to be a white-dominated space but talented women of colour and racialized persons have found space on the airwaves, particularly in our public broadcast sector (eg. Piya Chattopadhyay, Sook-Yin Lee, Matt Galloway and Candy Palmater of CBC Radio). In my attempt to acquire participants across the Toronto broadcast soundscape, thirty plus individual emails were sent to hosts, announcers and women led shows. Call outs were made across social media via Facebook and Twitter, but despite my continued attempts to diversify my sample to reflect Toronto's soundscape, my sample in the end lacked the diversity needed to address the experiences of racialized women in relation to their voice on the air. I am in discussion with fellow scholars, friends and the radio community to address the ethics and methods around creating a more equitable call for participation in any future research.

${ }^{7}$ Maurice Merleau-Ponty, The Primacy of Perception, Trans. by William Cobb (Evanston, IL: Northwestern University Press, 1964), 17.

${ }^{8}$ Steinar Kvale, "Introduction to Interview Research" In Doing Interviews, (SAGE Publications, Ltd, 2007), doi: 10.4135/9781849208963, 9. 
${ }^{9}$ Government of Canada, Tri Council Policy Statement (TCPS) 2: Ethical Conduct for Research Involving Humans, (2014), http:/www.pre.ethics.gc.ca/.

Conducting interviews with human subjects in Canada requires approval from the Research Ethics Board. Based on their three core principles "Respect for Persons, Concern for Welfare and Justice" (TCPS, Article 1.1).

${ }^{10}$ Ibid. As outlined in Article 10.4 of TCPS (2014), "where participants consent to have their identity disclosed, researchers shall record each participant's consent", I will made the option of anonymity "known to participants as part of the discussion around the nature and conditions of their consent."

${ }^{11}$ Christoph Maeder, "Analysing Sounds" in The SAGE Book of Qualitative Data Analysis, Edited by Uwe Flick (London: SAGE Publications, 2014), 430.

${ }^{12}$ Karpf, The Human Voice, (2007), 33.

Paralanguage as discussed by Karpf: "Pitch, loudness, and tempo - some of the features that make up paralanguage - can be more important than language itself. For paralanguage doesn't just support words but gives them life."

${ }^{13}$ Jeffery Pittam and Cynthia Gallois, "Predicting Impressions of Speakers from Voice Quality: Acoustic and Perceptual Measures" in The Journal of Language and Social Psychology, Vol 5, Issue 4, (July 2016), DOI: 10.1177/0261927X8600500401, 233-247.

Discussed further in chapter one, this study examined the relationship of an acoustic measure of speech based on the Long-Term Average Spectrum (LTAS), expert ratings of five voice qualities (breathy, creaky, nasal, tense, and whispery voices), and naive ratings of status and solidarity.

${ }^{14}$ Rosalind Gill, Gender and The Media, (Cambridge: Polity, 2007), 58.

${ }^{15}$ Ibid.

${ }^{16}$ Butler, Gender Trouble, $2^{\text {nd }}$ Ed. (1999), 171-90.

Performativity is not a singular act but a repetitive ritual. Butler outlines her theory of performativity in arguably her most notable work, though as stated in the preface to this tenth anniversary edition, much of her work following the publication of Gender Trouble "has been devoted to clarifying and revising the theory of performativity" that is outlined in this book (xiii).

${ }^{17}$ Rosalind Gill, "Discourse Analysis" In Qualitative Researching with Text, Image and Sound. (London: Sage, 2000), 222.

${ }^{18}$ Ibid, 224.

${ }^{19}$ Ibid.

${ }^{20}$ Kathy Charmaz, "Grounded Theory Methods in Social Justice" in The Sage Handbook of Qualitative Research, (Thousand Oaks, CA: Sage, 2011) 359-80.

${ }^{21}$ Ibid, 374.

${ }^{22}$ Ibid, 363. 
${ }^{23}$ Carla Willig, "Interpretation and Analysis" in The Sage Handbook of Qualitative Analysis, Edited by Uwe Flick (London: SAGE Publications, 2014).

${ }^{24}$ Will Styler, "Using Praat for Linguistic Research”, Ver. 1.7, (2017), http://savethevowels.org/praat/.

${ }^{25}$ Samantha Warhurst, Patricia McCabe, and Catherine Madill, "What Makes a Good Radio Voice: Perceptions of Radio Employers and Educators" in Journal of Voice, Vol. 27, no. 2, (2012), http://dx.doi.org/10.1016/j.jvoice.2012.08.010. $233-47$.

${ }^{26}$ Pittam and Gallois, "Predicting Impressions of Speakers from Voice Quality", (2016),

${ }^{27}$ Shelagh Davies and Joshua Goldberg, "Clinical Aspects of Transgender Speech Feminization and Masculinization" in International Journal of Transgenderism, vol. 9, (New York: Taylor and Francis Group, 2006), doi:10.1300/J485v09n03_08, 167-96.

${ }^{28}$ Walter Gershon, "Vibrational affect: Sound Theory and Practice in Qualitative Research" in Cultural Studies - Critical Methodologies, Vol. 13 no. 4, (London: SAGE Publications, 2013), 260.

${ }^{29}$ Ibid, 258.

${ }^{30}$ A. A. Berger, "Ethnomethodological Research" in Media and communication research methods: An introduction to qualitative and quantitative approaches, (Thousand Oaks, CA: Sage Publications, 2000), 151.

\section{Endnotes for Chapter Three: Ideal Radio Voice.}

${ }^{1}$ Karpf, "How Men and Women's Voices Are Changing (and Why)" in The Human Voice, (2007), 175.

${ }^{2}$ Philip Weston, Michael D. Hunter, Dilraj S. Sokhi, Iain D. Wilkinson, and Peter Woodruff, "Discrimination of voice gender in the human auditory cortex" in NeuroImage, no. 105, doi:10.1016/j.neuroimage.2014.10.056, (Elsevier B.V., 2015), 208-14.

${ }^{3}$ Samantha Warhurst, Patricia McCabe, and Catherine Madill, "What Makes a Good Voice for Radio: Perceptions of Radio Employers and Educators" in Journal of Voice, vol. 27, no. 2, (Elsevier B.V., Mar 2013), 217-24.

${ }^{4}$ Ibid. 221.

5 Julia De Laurentiis Johnson, "Radio \& Gendered Voice Semi-Structured Interview," Interview by author, (October 14, 2016).

${ }^{6}$ Stacey Englehart, "Radio \& Gendered Voice Semi-Structured Interview," Interview by author, (November 18, 2016).

${ }^{7}$ Susan McReynolds, "Radio \& Gendered Voice Semi-Structured Interview," Interview by author, (December 15, 2016). 
${ }^{8}$ Kat Callaghan, "Radio \& Gendered Voice Semi-Structured Interview," Interview by author, (October 21, 2016).

9 Elanor Grace, "Radio \& Gendered Voice Semi-Structured Interview," Skype interview by author. (October 30, 2016).

${ }^{10}$ Ibid.

${ }^{11}$ Dani Stover, "Radio \& Gendered Voice Semi-Structured Interview," Interview by author, (November 4, 2016).

${ }^{12}$ Kress, Gunther. "Language in the Media: The Construction of the Domains of Public and Private" in Media, Culture \& Society, vol 8, no. 4, doi:10.1177/0163443786008004003, (Oct 1986), 402.

${ }^{13}$ The subjects of authenticity and self-identity presented by these Toronto soundwork women are explored further in Chapter Five, "Radio Voice as Extension of Self: Vocal SelfIdentity and 'Authentic' Presentation".

${ }^{14}$ Christie Block, "Feminine Voice" in New York Speech and Voice Lab, (2014), http://www.nyspeechandvoicelab.net/transgender/voice-feminization/.

${ }^{15}$ The studies listed were used by Christine Block (2014) to create the pitch chart referenced here. Block kindly emailed me a list of sources they recommended on the subject of gendered voice from their expertise as a speech-language pathologist.

- Richard Adler, Sandy Hirsch, and Michelle Mordaunt, Voice and communication therapy for the transgender/transsexual client: a comprehensive clinical guide, (San Diego: Plural Pub., 2006). - Moya L. Andrews, "Reliability of student evaluations of voice therapy implications for theory and training" in Journal of Voice, vol. 13, no. 2, (1999), 227-33. doi:10.1016/s08921997(99)80025-3.

- Linda E. Spencer, "Speech Characteristics of Male-to-Female Transsexuals: A Perceptual and Acoustic Study" in Folia Phoniatrica et Logopaedica, vol. 40, no. 1 (1988), 31-42. doi:10.1159/000265881.

- Virginia I. Wolfe, David L. Ratusnik, Furman H. Smith, and Gretajo Northrop, "Intonation and Fundamental Frequency in Male-to-Female Transsexuals" in Journal of Speech and Hearing Disorders, vol. 55, no. 1 (1990), 43. doi:10.1044/jshd.5501.43.

${ }^{16}$ Shelagh, Davies and Joshua M. Goldberg. "Clinical Aspects of Transgender Speech Feminization and Masculinization" in International Journal of Transgenderism vol. 9, no. 3-4, (2006), 167-96. doi:10.1300/j485v09n03_08.

${ }^{17}$ Table 3.4 is reproduced here with permission from Block, "Chart" in New York Speech and Voice Lab, (2014), http://www.nyspeechandvoicelab.net/transgender/voice-feminization/.

${ }^{18}$ Englehart, "Radio \& Gendered Voice Semi-Structured Interview," (2016).

19 Stover, "Radio \& Gendered Voice Semi-Structured Interview," (2016).

${ }^{20}$ Maria Cristina Borrego, Gisele Gasparini, and Mara Behlau, "The Effects of a Specific Speech and Language Training Program on Students of a Radio Announcing Course" in Journal of Voice 21, no. 4 (2007), 426-32. 
${ }^{21}$ Ibid.

${ }^{22}$ Karpf, The Human Voice, (2007), 175.

${ }^{23}$ Stacey Thompson, "Radio \& Gendered Voice Semi-Structured Interview," Interview by author, (November 2, 2016).

${ }^{24}$ Siobhan Woodrow, "Radio \& Gendered Voice Semi-Structured Interview," Interview by author, (October 23, 2016).

${ }^{25}$ Englehart, "Radio \& Gendered Voice Semi-Structured Interview," (2016).

${ }^{26}$ Rosalind Gill, "Justifying Injustice: broadcasters accounts of inequality in radio" in Women \& Radio, edited by Caroline Mitchell, 137-51. (New York: Routledge, 2000), 148.

${ }^{27}$ Ihde, Listening and Voice, 2nd ed., (2007), 20.

${ }^{28}$ Butler, Gender Trouble, $10^{\text {th }}$ Anniv. Ed, (1999). 33.

\section{Endnotes for Chapter Four: Gendered Experience.}

${ }^{1}$ Hilmes, Radio Voices, (1997). See chapter on the Disembodied Woman.

${ }^{2}$ Ibid.

${ }^{3}$ Mitchell. Women and Radio: Airing Differences, (London: Routledge, 2000).

${ }^{4}$ Christine Ehrick, Radio and the Gendered Soundscape, (2015).

${ }^{5}$ Mitchell, “Introduction: On a Women's Wavelength?” in Women and Radio, (2000), 5.

${ }^{6}$ Butler, Gender Trouble, $2^{\text {nd }}$ Ed., (1999). Butler delves into the politics of gender and power 33-44.

${ }^{7}$ Walter Ong, Orality and Literacy: the technologizing of the word. (London: Routledge, 1982), 32 .

${ }^{8}$ Adriana Cavarero, "Part III: A Politics of Voices" in For More than One Voice, Translated by Paul A. Kottman, (Standford, CA: Stanford University Press, 2005), 163-212.

${ }^{9}$ Hannah Arendt, The Human Condition (Chicago: University of Chicago Press, 1958), 3.

${ }^{10}$ Caverero, "Part III: A Politics of Voices", (2005), 209.

${ }^{11}$ Joan W. Scott, "The Evidence of Experience" in Critical Inquiry, vol. 17, no. 4, (Summer 1991), 773-97. http://www.jstor.org/stable/1343743.

${ }^{12}$ Rosalind Gill, "Justifying Injustice: broadcasters accounts of inequality in radio" in Women and Radio, edited by Caroline Mitchell, (Routledge, 2000), 137-51. 
${ }^{13}$ The Trumpian Era (following the election and inauguration of American President Donald Trump in 2016) has sparked global protests and a surge in feminist politics across the globe. In 2017, feminism still proves to be controversial but the election of Trump ignited a feminist backlash with the "Women's March on Washington" January $21^{\text {st }}, 2017$ along with united marches in solidarity around the globe from Toronto, Canada to London, England. See K.J. Baker "We Still Need Feminism" in Women in Higher Education, Vol. 25, no. 12, (December 2016), 20, doi:10.1002/whe.20388. See also Dubravka Zarkov, "Women, Feminism and Politics" in European Journal of Women's Studies, Vol 24, no. 1, (Jan 2017), 3-6, DOI $10.1177 / 1350506816681124$.

${ }^{14}$ Kat Callaghan, "Radio \& Gendered Voice Semi-Structured Interview," Interview by author, (October 21, 2016).

${ }^{15}$ Amy Heckerling, Clueless, Movie, Directed by Amy Heckerling (United States: Paramount Pictures, 1995). Clueless portrays the valley girl image not only through visuals but speech and language as noted in Jennifer O'Meara. "“We've Got to Work on Your Accent and Vocabulary": Characterization through Verbal Style in Clueless" in Cinema Journal vol 53, no. 3 (2014), 138-145. https://muse.jhu.edu/.

${ }^{16}$ Siobhan Woodrow, "Radio \& Gendered Voice Semi-Structured Interview," Interview by author, (October 23, 2016).

${ }^{17}$ See "From Upspeak to Vocal Fry: Are We 'Policing' Young Women's Voices?" on Fresh Air, NPR Radio Show, (July 23, 2015) and "The unstoppable march of the upward inflection?" BBC News Article. (August 11, 2014). See also, Ira Glass, "545: If You Don't Have Anything Nice to Say, SAY IT IN ALL CAPS," from This American Life, Podcast, (January 23, 2015).

${ }^{18}$ Paul Warren, Uptalk: The Phenomenon of Rising Intonation, (Cambridge: Cambridge University Press, 2016), doi:10.1017/CBO9781316403570.

${ }^{19}$ Thomas J. Linneman, "Gender in Jeopardy!" in Gender \& Society vol. 27, no. 1 (2013), 82-105. doi:10.1177/0891243212464905.

${ }^{20}$ Joseph C Tyler, "Expanding and Mapping the Indexical Field" in Journal of English Linguistics 43, no. 4 (2015): 284-310. doi:10.1177/0075424215607061., 294.

${ }^{21}$ Mark Liberman, "The phonetics of uptalk" in Language Log, (September 2008), http://languagelog.ldc.upenn.edu/nll/?p=586. Also see Warren, "Uptalk in English varieties" in Uptalk, 68-96, (2016). Discussion of Canadian English by Warren is included in the noted chapter.

${ }^{22}$ E. James, C. Mahut, and G. Latkiewitcz, "The investigation of an apparently new intonation pattern in Toronto English", in Information and Communication, vol. 10, 11-17, (1989), 16.

${ }^{23}$ Jianjing Kuang and Mark Liberman, "The effect of vocal fry on pitch perception" in 2016 IEEE International Conference on Acoustics, Speech and Signal Processing, (2016).

${ }^{24}$ Lesley Wolk, Nassima B. Abdelli-Beruh, and Dianne Slavin, "Habitual Use of Vocal Fry in Young Adult Female Speakers" in Journal of Voice 26, no. 3 (2012). 
${ }^{25}$ Rindy C. Anderson, Casey A. Klofstad, William J. Mayew, and Mohan Venkatachalam. "Vocal Fry May Undermine the Success of Young Women in the Labor Market" in PLoS ONE 9, no. 5 (2014). doi:10.1371/journal.pone.0097506.

${ }^{26}$ Ira Glass, "545: If You Don't Have Anything Nice to Say, SAY IT IN ALL CAPS," from This American Life, Podcast, (January 23, 2015).

${ }^{27}$ Katie Hill. "Radio \& Gendered Voice Semi-Structured Interview," Interview by author, (October 28, 2016).

${ }^{28}$ Callaghan, "Radio \& Gendered Voice Semi-Structured Interview," (2016).

${ }^{29}$ Ehrick, Radio and the gendered soundscape (2015), 14.

${ }^{30}$ Dani Stover, "Radio \& Gendered Voice Semi-Structured Interview," Interview by author, (November 4, 2016).

${ }^{31}$ Caroline Mitchell, "Sound advice for women who want to work in radio" in Women and Radio, 262-274, (2000), 265.

${ }^{32}$ Anne McKay “Speaking up: voice amplification and women's struggle for public expression" in Women and Radio, (2000), 15-28.

${ }^{33}$ Ibid.

${ }^{34}$ Walter J. Ong, "Agonistic structures in academia: Past to present" in Interchange vol. 5 , no. 4, 1-12, (1974), 8.

${ }^{35}$ McKay "Speaking up: voice amplification and women's struggle for public expression" (2000), 22 .

${ }^{36}$ Stacey Thompson, "Radio \& Gendered Voice Semi-Structured Interview," Interview by author, (November 2, 2016).

${ }^{37}$ Susan McReynolds, "Radio \& Gendered Voice Semi-Structured Interview," Interview by author, (December 15, 2016).

${ }^{38}$ Julia De Laurentiis Johnson, "Radio \& Gendered Voice Semi-Structured Interview," Interview by author, (October 14, 2016).

${ }^{39}$ Kat Callaghan, "Radio \& Gendered Voice Semi-Structured Interview," Interview by author, (October 21, 2016).

${ }^{40}$ \#Gamergate targeted several women in the video game industry, including game developers Zoë Quinn and Brianna Wu. The controversy began in in August 2014 and sparked concerns over sexism and misogyny in video game culture. For feminist work on trolling, sexism in gaming and gamergate, see Shira Chess and Adrienne Shaw, "A Conspiracy of Fishes, Or, how we Learned to Stop Worrying about \#GamerGate and Embrace Hegemonic Masculinity," in Journal of Broadcasting \& Electronic Media vol. 59 no. 1, (2015), 208-220. For trolling see also 
Mathew Scheier, "Mary Beard and her Battle Cry Against Internet Trolling" in NY Times, Article, (April 16, 2016).

${ }^{41}$ Hilmes, "The New Materiality of Radio." In Radio's new wave: global sound in the digital era, by Jason Loviglio and Michele Hilmes. New York: Routledge, Taylor \& Francis Group, 2013.

${ }^{42}$ Stover, "Radio \& Gendered Voice Semi-Structured Interview" in Interview by author, (November 4, 2016).

${ }^{43}$ Hilmes, Radio voices, (1997), 137.

${ }^{44}$ Ibid, 18. Hilmes discusses how women in early broadcast were perceived as a threat to the high-culture radio image in America.

${ }^{45}$ Ibid, 283.

${ }^{46}$ Ibid, 283.

${ }^{47}$ Siobhan Woodrow, "Radio \& Gendered Voice Semi-Structured Interview" in Interview by author, (October 23, 2016).

${ }^{48}$ Ibid.

${ }^{49}$ Based on Canadian report from "Toronto Topline Radio" in Numeris PPM Statistics, (November 27, 2016), http://assets.numeris.ca/Downloads/2016-

17_12_Radio_ME_TorontoToplineRadio.pdf.

${ }^{50}$ Analysis is taken from posted data as of February 2017, show hosts may change before the publication of this work.

${ }^{51}$ As a publically funded broadcaster, CBC Radio reflects a mandate of diversity which includes gender equity. This can be seen in their number of women on air in lead roles. Eg. Fresh Air, CBC Radio 2 Morning, CBC Radio 2 Weekend Mornings, and The Current. See "Mandate of CBC/Radio-Canada," CBC/Radio-Canada, (Accessed April 02, 2017), http:/www.cbc.radiocanada.ca/en/explore/mandate/.

${ }^{52}$ Statistics Canada, "The surge of women in the workforce" in Canadian Megatrends, (March 2017), http://www.statcan.gc.ca/pub/11-630-x/11-630-x2015009-eng.htm.

${ }^{53}$ Erin Phillips and Paul Phillips, "Women's Work in Canada, The Historical Perspective" in Women \& Work: inequality in the Canadian labour market, (Toronto: James Lorimer, 2000), 9-16.

${ }^{54}$ Kate Puppatz, "Gender, Class, Work and Reworking Bourdieu" in Gender Capital at Work, (London: Palgrave Macmillan UK, 2012). In the introduction to Part I of their book on the intersections of femininity, masculinity, class and occupation, Huppatz notes that women in contemporary western society continue to cluster in occupations such as nursing, teaching, social work, retail work and child care. Huppatz argues that despite increased numbers of women in the workplace (post-1990), certain occupations are becoming even more gendered, with feminized jobs carrying significant pay inequality compared to masculinized jobs. 
${ }^{55}$ Woodrow, "Radio \& Gendered Voice Semi-Structured Interview" in Interview by author, (October 23, 2016).

${ }^{56}$ Ihde, "The Polyphony of Experience" in Listening and Voice (2007), 117.

57 " Ibid, 118.

${ }^{58}$ Cavarero, For More Than One Voice (2005), 522.

${ }^{59}$ Schlichter, "Do Voices Matter? Vocality, Materiality, Gender Performativity." Body \& Society 17, no. 1, 31-52, (2011), 43.

\section{Endnotes for Chapter Five: Extension of Self.}

${ }^{1}$ Hilmes, "The Disembodied Woman" in Radio Voices, (1999), 130-50.

Hilmes draws on the notion of the disembodied voice to discuss the historical biases against women's voices on early American radio.

${ }^{2}$ Cavarero, For More Than One Voice, trans. Paul A. Kottman (2005), 522.

${ }^{3}$ Erik Piepenburg, "How to Master an Accent" in The New York Times, (November 23, 2016), https://www.nytimes.com/2016/11/23/theater/how-to-master-an-accent.html.

${ }^{4}$ NPR. "Playing with Perceptions." In TED Radio Hour (audio blog), (November 14, 2014), http://www.npr.org/programs/ted-radio-hour

${ }^{5}$ Thornham, Women, Feminism and Media, (2010), 107.

${ }^{6}$ Walter Sendlmeier, "Introduction: Voice - Emotion - Personality” In Electrified Voices: medial, socio-historical and cultural aspects of voice transfer. Editors Zakharine, Dmitri, and Nils Meise. (Göttingen: Niedersachs, 2013), 32.

${ }^{7}$ Woodrow. "Radio \& Gendered Voice Semi-Structured Interview." Interview by author. (October 23, 2016).

${ }^{8}$ Ibid.

9 John A. Haskell, "Vocal self-perception: The other side of the equation" in Journal of Voice (1, no. 2 1987), 172. doi:10.1016/s0892-1997(87)80042-5.

${ }^{10}$ Katie Hill, "Radio \& Gendered Voice Semi-Structured Interview." Interview by author. (October 28, 2016).

${ }^{11}$ Dani Stover, "Radio \& Gendered Voice Semi-Structured Interview." Interview by author. (November 4, 2016).

${ }^{12}$ Barry Truax "Voices in the Soundscape: From Cellphones to Soundscape Composition" in Electrified Voices, (2013) 62. 
${ }^{13}$ Frances Dyson, "The Geneology of The Radio Voice" in Radio Rethink: art, sound and transmission, (Banff, Alta: Walter Phillips Gallery, 1994), 178.

${ }^{14}$ Morwenna Griffiths, Feminisms and the self: the web of identity, (London: Routledge, 1995), 173.

${ }^{15}$ Lionel Trilling, Sincerity and Authenticity, (Harvard University Press, 1972), 11.

${ }^{16}$ Griffiths, Feminisms and the self: the web of identity, (1995), 169.

17 Matthis Drolet, Ricarda I. Schubotz, and Julia Fischer, "Authenticity affects the recognition of emotions in speech: behavioral and fMRI evidence" in Cognitive, Affective, \& Behavioral Neuroscience 12(2011), 140-50. doi:10.3758/s13415-011-0069-3.

${ }^{18}$ Julia De Laurentiis Johnson. "Radio \& Gendered Voice Semi-Structured Interview." Interview by author. (October 14, 2016).

${ }^{19}$ Eleanor Grace, "Radio \& Gendered Voice Semi-Structured Interview" Online interview by author, (October 30, 2016).

${ }^{20}$ Siobhan Woodrow. "Radio \& Gendered Voice Semi-Structured Interview." Interview by author. (October 23, 2016).

${ }^{21}$ Ibid.

${ }^{22}$ Ibid.

${ }^{23}$ Butler, Gender Trouble, $2^{\text {nd }}$ Ed. (1999), 179. Butler argues, "gender is an identity tenuously constituted in time, instituted in an exterior space through a stylized repetition of acts."

${ }^{24}$ Benhabib, Situating the Self: gender, community and postmodernism in contemporary ethics, (Oxford: Polity Press, 1992), 215.

${ }^{25}$ Siobhan Woodrow. "Radio \& Gendered Voice Semi-Structured Interview." Interview by author. (October 23, 2016).

${ }^{26}$ Ibid.

${ }^{27}$ Trilling, Sincerity and Authenticity, (1972), 131.

${ }^{28}$ Dani Stover, "Radio \& Gendered Voice Semi-Structured Interview." Interview by author. (November 4, 2016).

${ }^{29}$ Truax "Voices in the Soundscape: From Cellphones to Soundscape Composition" in Electrified Voices, (2013) 69.

${ }^{30}$ Ibid, 69.

${ }^{31}$ James Wolcott, "So, Like, Why Are We So Obsessed with Podcasts Right Now?" in Vanity Fair, (January 08, 2016). http://www.vanityfair.com/culture/2016/01/james-wolcott-onpodcasts. 
From Vanity Fair to the halls of academia, podcasting has burst forward from the depths of the Internet into a main media staple. Many attribute the rise in podcast interest to the success of the true crime series Serial in 2014 hosted by Sarah Koenig of This American Life (NPR).

${ }^{32}$ Marshall McLuhan, Understanding Media: The Extensions of Man, (London: Sphere, 1967), 318.

${ }^{33}$ Ibid. 322.

${ }^{34}$ Stover, "Radio \& Gendered Voice Semi-Structured Interview." Interview by author. (November 4, 2016).

${ }^{35}$ Woodrow. "Radio \& Gendered Voice Semi-Structured Interview." Interview by author. (October 23, 2016).

${ }^{36}$ Ibid.

${ }^{37}$ Ibid.

${ }^{38}$ R. Murray Schafer, The soundscape: the tuning of the world. (Rochester, VT: Destiny Books 1993), 235.

\section{Endnotes for Conclusion}

${ }^{1}$ Cavarero, For More Than One Voice (2005), 522.

${ }^{2}$ Anne Karpf, (2007), 27.

${ }^{3}$ Butler, Gender Trouble, $10^{\text {th }}$ Anniv. Ed, (1999). 33.

${ }^{4}$ Hilmes, "Disembodied Woman" in Radio Voices, (1997), 130-50.

Hilmes disembodied woman chapter notes early experiences of gender bias in the radio industry.

${ }^{5}$ Rosalind Gill (2000)

${ }^{6}$ Butler, Gender Trouble, $10^{\text {th }}$ Anniversary Ed., (1999). Butler delves into the politics of gender and power 33-44.

${ }^{7}$ Caverero, "Part III: A Politics of Voices", (2005), 209.

${ }^{8}$ Thornham, Women, Feminism and Media, (2010), 107.

${ }^{9}$ Schlichter, "Do Voices Matter? Vocality, Materiality, Gender Performativity." Body \& Society 17, no. 1, 31-52, (2011), 43.

${ }^{10}$ Mentoring Inspiring Women in Radio, MIW Gender Analysis Study (February 2017), http://www.radiomiw.com/images/PDFs/2016_GenderAnalysisFINAL.pdf.

${ }^{11}$ WACC, "Canada National Report”, in Global Media Monitoring Project 2015, (2016), http://cdn.agilitycms.com/who-makes-the-news/Imported/reports_2015/national/Canada.pdf. 
${ }^{12}$ Bob Papper, "RTDNA Research: Women and minorities in newsrooms," Newsroom Research Project, http://rtdna.org/article/rtdna_research_women_and_minorities_in_newsrooms. The term "visible minorities" is used to match the terms used in Papper's report.

${ }^{13}$ Amira Elghawaby, "The Invisibility of Canada's Diversity," in New Canadian Media, (February $\left.25^{\text {th }}, 2014\right)$, http://www.newcanadianmedia.ca/item/12746-the-invisibility-of-canadasdiversity/.

${ }^{14}$ Ibid.

${ }^{15}$ For work on sound and race, see Stoever, The Sonic Color Line (2016). See also Art M. Blake, "Audible Citizenship and Audiomobility: Race, technology, and CB radio", in American Quarterly vol. 63:3 (September 2011). See also Gustavus Stadler, "On Whiteness and Sound Studies”, in Sounding Out! Blog (July 2015), soundstudiesblog.com/2015/07/06/on-whitenessand-sound-studies/.

${ }^{16}$ Stoever, The Sonic Color Line, (2016), 21.

${ }^{17}$ Woodrow, "Radio \& Gendered Voice Semi-Structured Interview," Interview by author, (October 23, 2016).

${ }^{18}$ Ibid. 


\section{Bibliography}

\section{Interviews}

Callaghan, Kat. "Radio \& Gendered Voice Semi-Structured Interview." Interview by author. October 21, 2016.

De Laurentiis Johnson, Julia. "Radio \& Gendered Voice Semi-Structured Interview." Interview by author. October 14, 2016.

Englehart, Stacey. "Radio \& Gendered Voice Semi-Structured Interview." Interview by author. November 18, 2016.

Grace, Eleanor. "Radio \& Gendered Voice Semi-Structured Interview." Online interview by author. October 30, 2016.

Hill, Katie. "Radio \& Gendered Voice Semi-Structured Interview." Interview by author. October 28, 2016.

Laur, Kattie. "Radio \& Gendered Voice Semi-Structured Interview." Interview by author. November 5th, 2016.

McReynolds, Susan. "Radio \& Gendered Voice Semi-Structured Interview." Interview by author. December 15, 2016.

Stover, Dani. "Radio \& Gendered Voice Semi-Structured Interview." Interview by author. November 4, 2016.

Thompson, Stacey. "Radio \& Gendered Voice Semi-Structured Interview." Interview by author. November 2, 2016.

Woodrow, Siobhan. "Radio \& Gendered Voice Semi-Structured Interview." Interview by author. October 23, 2016.

\section{Books, Articles and Other Materials}

Adler, Richard Kenneth., Sandy Hirsch, and Michelle Mordaunt. Voice and communication therapy for the transgender/transsexual client: a comprehensive clinical guide. San Diego: Plural Pub., 2006.

Allen Amy. The Politics of Our Selves: power, autonomy, and gender in contemporary critical theory. New York: Columbia University Press, 2008.

Anderson, Rindy C., Casey A. Klofstad, William J. Mayew, and Mohan Venkatachalam. "Vocal Fry May Undermine the Success of Young Women in the Labor Market." PLoS ONE 9, no. 5 (2014). doi:10.1371/journal.pone.0097506.

Andrews, Moya L. "Reliability of student evaluations of voice therapy implications for theory and training." Journal of Voice 13, no. 2 (1999): 227-33. doi:10.1016/s0892-1997(99)80025-3.

Arendt, Hannah. The Human Condition. Chicago: University of Chicago Press, 1958.

Baker K.J.. "We Still Need Feminism." Women in Higher Education, Vol. 25, no. 12, (December 2016): 20. doi:10.1002/whe.20388

Barthes, Roland. Image, music, text, trans. by S. Heath. New York: Hill \& Wang, 1977.

Barthes, Roland. The grain of the voice: interviews 1962-1980. New York: Hill and Wang, 1985.

Benhabib, Seyla. Situating The Self: gender, community and postmodernism in contemporary ethics. New York: Routledge, 1992.

Benjamin, Walter. The work of art in the age of mechanical reproduction. London: Penguin, 2008. 
Beauvoir, Simone de. The Second Sex, trans. by H.M Parshley, New York: Alfred A. Knopf, 1975.

Blake, Art M. "Audible Citizenship and Audiomobility: Race, technology, and CB radio", American Quarterly vol. 63:3 (September 2011).

Block, Christie. "Feminine Voice." New York Speech and Voice Lab. 2014. Accessed January 28, 2017. http://www.nyspeechandvoicelab.net/transgender/voicefeminization/.

Borrego, Maria Cristina, Gisele Gasparini, and Mara Behlau. "The Effects of a Specific Speech and Language Training Program on Students of a Radio Announcing Course." Journal of Voice 21, no. 4 (2007): 426-32. doi:10.1016/j.jvoice.2006.03.003.

Butler, Judith. Gender trouble: feminism and the subversion of identity. New York: Routledge, 1990.

Cavarero, Adriana, and Paul A. Kottman. For more than one voice: toward a philosophy of vocal expression. Stanford, CA: Stanford University Press, 2005.

Charmaz, Kathy. "Grounded Theory Methods in Social Justice." In The Sage Handbook of Qualitative Research, Thousand Oaks, CA: Sage, 2011. 359-80.

Chess, Shira and Adrienne Shaw, "A Conspiracy of Fishes, Or, how we Learned to Stop Worrying about \#GamerGate and Embrace Hegemonic Masculinity." Journal of Broadcasting \& Electronic Media vol. 59 no. 1, (2015), 208-220. doi:10.1080/08838151.2014.999917.

Davies, Shelagh, and Joshua M. Goldberg. "Clinical Aspects of Transgender Speech Feminization and Masculinization." International Journal of Transgenderism 9, no. 3-4 (2006): 167-96. doi:10.1300/j485v09n03 08.

Dyson, Frances. "The Genealogy of the Radio Voice." In Radio Rethink: art, sound and transmission, edited by Daina Augaitis and Dan Lander, 167-88. Banff, Canada: Walter Phillips Gallery, 1994.

Ehrick, Christine. Radio and the gendered soundscape: women and broadcasting in Argentina and Uruguay, 1930-1950. New York, NY: Cambridge University Press, 2015.

Fisher Linda. "Feminist Phenomenological Voices." In Continental Philosophy Review, no. 43: 83-95. April 2010. DOI 10.1007/s11007-010-9132-y.

Foucault, Michele. Madness and Civilization: a history of insanity in the Age of Reason, New York: Vintage Books, 1988.

Foucault, Michele. "Preface to The History of Sexuality, Volume II" in The Foucault Reader, edited by Paul Rabinow, New York: Vintage Books, 2010.

Gershon, Walter. "Vibrational affect: Sound Theory and Practice in Qualitative Research.” In Cultural Studies - Critical Methodologies, Vol. 13 no. 4, London: SAGE Publications, 2013.

Gill, Rosalind. "Justifying Injustice: broadcasters accounts of inequality in radio." In Women \& Radio, edited by Caroline Mitchell, 137-51. New York: Routledge, 2000 .

Glass, Ira. "545: If You Don't Have Anything Nice to Say, SAY IT IN ALL CAPS." This American Life. January 23, 2015. Accessed February 01, 2017. https://www.thisamericanlife.org/radio-archives/episode/545/transcript. 
Griffiths, Morwenna. Feminism and the Self: The Web of Identity. London: Routledge, 2003.

Halper, Donna. "Speaking for Themselves: How Radio Brought Women into The Public Sphere." Radio Cultures: the sound medium in american life, Edited by Michael C. Keith.

Heidegger, Martin. Being and Time, trans. by Joan Stambaugh, Albany, NY: State University of New York Press, 1996.

Hilmes, Michele. "The New Materiality of Radio." Radio's new wave: global sound in the digital era, by Jason Loviglio and Michele Hilmes. New York: Routledge, Taylor \& Francis Group, 2013.

Hilmes, Michele. Radio voices: American broadcasting, 1922-1952. Minneapolis, MN: University of Minnesota Press, 1997.

Husserl, Edmund. The Crisis of European Sciences and Transcendental Phenomenology, Evanston, IL: Northwestern University Press, 1970.

Ihde, Don. Listening and Voice: Phenomenologies of Sound, 2nd Ed. New York: SUNY Press, 2007.

Karpf, Anne. The human voice: the story of a remarkable talent. London: Bloomsbury, 2006.

Kvale, Steinar. "Introduction to Interview Research" In Doing Interviews, SAGE Publications, Ltd, 2007. doi: 10.4135/9781849208963.

Kimbrough, Andrew. Dramatic theories of voice in the twentieth century. Amherst, NY: Cambria Press, 2011.

Kress, Gunther. "Language in the Media: The Construction of the Domains of Public and Private." Media, Culture \& Society 8, no. 4 (1986): 395-419. doi:10.1177/0163443786008004003.

Kuang, Jianjing, and Mark Liberman. "The effect of vocal fry on pitch perception." 2016 IEEE International Conference on Acoustics, Speech and Signal Processing (ICASSP), 2016. doi:10.1109/icassp.2016.7472681.

Lacey, Kate. Feminine Frequencies: Gender, german radio, and the Public Sphere, 1923-1945. Michigan: University of Michigan Press, 1997.

Linneman, Thomas J. "Gender in Jeopardy!" Gender \& Society 27, no. 1 (2013): 82-105. doi: $10.1177 / 0891243212464905$.

Loviglio, Jason. "Sound effects: Gender, voice and the cultural work of NPR." Radio Journal: International Studies in Broadcast \& Audio Media 5, no. 2. 67-81. 2007. doi:10.1386/rajo.5.2-3.67_1.

Madsen, Virginia and John Potts. "Voice-Cast: The Distribution of the Voice via Podcasting." Voice: Vocal aesthetics in digital arts and media, 33-59. Cambridge, Mass: MIT Press, 2010.

Maeder, Christoph. "Analysing Sounds." The SAGE Book of Qualitative Data Analysis, Edited by Uwe Flick. London: SAGE Publications, 2014.

McKay, Anne. "Speaking Up: Voice Amplification and Women's Struggle for Public Expression." Women and Radio: Airing Differences, edited by Caroline Mitchell. London: Routledge, 2000.

McLuhan, Marshall. Understanding media: the extensions of man. New York: McGrawHill, 1964. 
Merleau-Ponty, Maurice. The Primacy of Perception, and other essays on phenomenology of psychology, the philosophy of art, history and politics, Edited by James M. Edie. Evanston, Il: Northwestern University Press, 1964.

Mitchell, Caroline. Women and Radio: airing differences. London: Routledge, 2000.

Neumark, Norie, Ross Gibson, and Theo Van Leeuwen. Voice: vocal aesthetics in digital arts and media. Cambridge, MA: MIT Press, 2010.

O’Meara, Jennifer. "“We've Got to Work on Your Accent and Vocabulary": Characterization through Verbal Style in Clueless." Cinema Journal 53, no. 3 (2014): 138-145. https://muse.jhu.edu/ (accessed March 26, 2017).

Ong, Walter J. "Agonistic structures in academia: Past to present." Interchange 5, no. 4 (1974): 1-12. doi:10.1007/bf02144923.

Ong, Walter J. Orality and literacy: the technologizing of the word. London: Routledge, 1982.

Phillips, Erin and Paul Phillips, Women \& Work: inequality in the Canadian labour market. Toronto: James Lorimer, 2000.

Pittam, J., and C. Gallois. "Predicting Impressions of Speakers from Voice Quality: Acoustic and Perceptual Measures." Journal of Language and Social Psychology 5, no. 4 (1986): 233-47. doi:10.1177/0261927x8600500401.

Scannell, Paddy. "Mass Media, Mass Democracy." The Making of Britain, 1988, 99-112. doi:10.1007/978-1-349-19180-2 8.

Scannell, Paddy. Radio, Television and Modern Life, Oxford: Blackwell, 1996.

Schaeffer, Pierre. Traité des objects musicaux. Paris: Editions du Seuil, 1966.

Schafer, R. Murray. The soundscape: our sonic environment and the tuning of the world. Rochester, VT: Destiny Books, 1993.

Schlichter, Annette. "Do Voices Matter? Vocality, Materiality, Gender Performativity." Body \& Society 17, no. 1 (2011): 31-52. doi:10.1177/1357034x10394669.

Scott, Joan W. "The Evidence of Experience." Critical Inquiry 17, no. 4 (1991): 773-97. doi:10.1086/448612.

Seaton, Matt. "Speech habits: Uptalk." The Guardian. September 21, 2001. Accessed February 01, 2017.

https://www.theguardian.com/books/2001/sep/21/referenceandlanguages.mattsea ton.

Spencer, Linda E. "Speech Characteristics of Male-to-Female Transsexuals: A Perceptual and Acoustic Study." Folia Phoniatrica et Logopaedica 40, no. 1 (1988): 31-42. doi:10.1159/000265881.

Stadler, Gustavus. “On Whiteness and Sound Studies.” Sounding Out! Blog, (July 2015). soundstudiesblog.com/2015/07/06/on-whiteness-and-sound-studies/.

Stoever, Jennifer Lynn. The Sonic Color Line: Race and the cultural politics of listening, New York: NYU Press, 2016.

Thornham, Sue. Women, feminism and media, Edinburgh: Edinburgh Univ. Press, 2010.

Tyler, Joseph C. "Expanding and Mapping the Indexical Field." Journal of English Linguistics 43, no. 4 (2015): 284-310. doi:10.1177/0075424215607061.

Valentine, Carol Ann, and Banisa Saint Damian. "Gender and culture as determinants of the 'ideal voice'." Semiotica 71, no. 3-4 (1988). doi:10.1515/semi.1988.71.34.285 . 
Vaillant, Derek W. "Sounds of Whiteness: Local radio, racial formation, and public culture in Chicago, 1921-1935", in American Quarterly 54.1, 25-66 (March 2002).

Walsh, Kathy, Jane Suiter and Orla O'Connor, Warhurst, Samantha, Patricia Mccabe, and Catherine Madill. "What Makes a Good Voice for Radio: Perceptions of Radio Employers and Educators." Journal of Voice 27, no. 2 (2013): 217-24. doi:10.1016/j.jvoice.2012.08.010.

Warhurst, Samantha, Patricia McCabe, and Catherine Madill. 2013. "What Makes a Good Voice for Radio: Perceptions of Radio Employers and Educators." Journal of Voice 27 (2): 217-24.

Warren, Paul. Uptalk: The Phenomenon of Rising Intonation. Cambridge: Cambridge University Press, 2016. doi:10.1017/CBO9781316403570.

Weston, Philip S.j., Michael D. Hunter, Dilraj S. Sokhi, Iain D. Wilkinson, and Peter W.r. Woodruff. "Discrimination of voice gender in the human auditory cortex." NeuroImage 105 (2015): 208-14. doi:10.1016/j.neuroimage.2014.10.056.

Wolfe, Virginia I., David L. Ratusnik, Furman H. Smith, and Gretajo Northrop. "Intonation and Fundamental Frequency in Male-to-Female Transsexuals." Journal of Speech and Hearing Disorders 55, no. 1 (1990): 43. doi:10.1044/jshd.5501.43.

Wolk, Lesley, Nassima B. Abdelli-Beruh, and Dianne Slavin. "Habitual Use of Vocal Fry in Young Adult Female Speakers." Journal of Voice 26, no. 3 (2012). doi:10.1016/j.jvoice.2011.04.007.

Zakharine, Dmitri and Nils Meise. Electrified Voices: Medial, Socio-historical and Cultural Aspects of Voice Transfer, V\&R Unipress, 2013.

Zarkov, Dubravka. "Women, Feminism and Politics." European Journal of Women's Studies, Vol 24, no. 1, (Jan 2017), 3-6. DOI 10.1177/1350506816681124. 